University of Louisville

ThinkIR: The University of Louisville's Institutional Repository

Electronic Theses and Dissertations

$12-2010$

\title{
Leadership, curriculum, instruction, and accountability scores : evidence from Kentucky scholastic audits.
}

Rebecca Curry Todd

University of Louisville

Follow this and additional works at: https://ir.library.louisville.edu/etd

\section{Recommended Citation}

Todd, Rebecca Curry, "Leadership, curriculum, instruction, and accountability scores : evidence from Kentucky scholastic audits." (2010). Electronic Theses and Dissertations. Paper 1447.

https://doi.org/10.18297/etd/1447

This Doctoral Dissertation is brought to you for free and open access by ThinkIR: The University of Louisville's Institutional Repository. It has been accepted for inclusion in Electronic Theses and Dissertations by an authorized administrator of ThinkIR: The University of Louisville's Institutional Repository. This title appears here courtesy of the author, who has retained all other copyrights. For more information, please contact thinkir@louisville.edu. 


\title{
LEADERSHIP, CURRICULUM, INSTRUCTION, AND ACCOUNTABILITY SCORES: EVIDENCE FROM KENTUCKY SCHOLASTIC AUDITS
}

\author{
By \\ Rebecca Curry Todd \\ B.A., Western Kentucky University \\ B.S., Western Kentucky University \\ M.A.Ed, Western Kentucky University

\begin{abstract}
A Dissertation
Submitted to the Faculty of the

Graduate School of the University of Louisville

and

Graduate Studies and Research at Western Kentucky University

In Partial Fulfillment of the Requirements

for the Degree of
\end{abstract} \\ Doctor of Philosophy \\ Department of Leadership, Foundations, and Human Resource Education \\ University of Louisville \\ and \\ College of Education and Behavioral Sciences \\ Western Kentucky University
}

December 2010 


\title{
LEADERSHIP, CURRICULUM, INSTRUCTION, AND ACCOUNTABILITY SCORES: EVIDENCE FROM KENTUCKY SCHOLASTIC AUDITS
}

\author{
By
}

\author{
Rebecca Curry Todd \\ B.A., Western Kentucky University \\ B.S., Western Kentucky University \\ M.A., Western Kentucky University
}

A Dissertation Approved on

November 23, 2010

by the following Dissertation Committee:

Drssertation Co-Chair

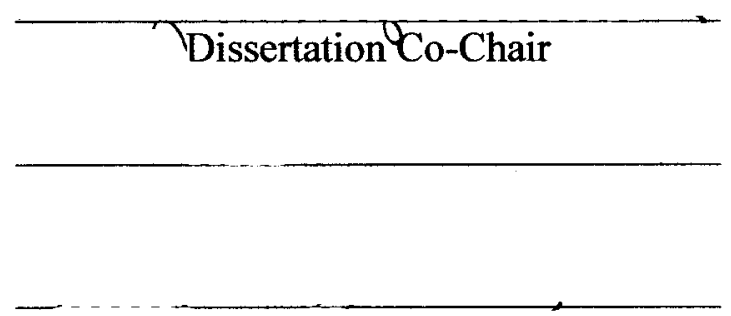




\title{
DEDICATION
}

This dissertation is dedicated to

\author{
God \\ (my Heavenly Father)
}

whose mercy and grace make all things possible;

\author{
Brad Todd \\ (my dear husband) \\ who I owe (and give) my love, loyalty, and gratitude;
}
Rev. Stanley T. Curry (deceased) and Mrs. Betty Curry
(my parents)

who instilled in me the value of an education;

\section{Christopher, Kristen, and Kyle \\ (my children)}

who I love with all my heart. You and Brad are the wind beneath my wings!

Beverly, Patty, Shelia, Sherry, Stan, and Tammy Jo

(my sisters and brother)

I love you! 


\section{ACKNOWLEDGMENTS}

Dr. Stephen Miller, University of Louisville and Western Kentucky University, dissertation co-chair, your support and patience throughout the doctoral experience, especially the dissertation process, are the contributions making this terminal degree a reality. Without you, this would not be. Thank you.

Dr. Douglas Smith, Western Kentucky University, co-chair and methodologist, is responsible for the methodology of this study. His expertise in statistical analysis defined the results of this study and its completion.

Dr. Gayle Ecton, Western Kentucky University, dissertation committee member and graduate advisor, thank you for staying the course with me throughout my graduate work experiences, Master's degree through Doctorate of Philosophy. Dr. Blake Haselton, University of Louisville, dissertation committee member, thank you for being kind and encouraging, for your insights as a former superintendent. And, Dr. Bob Ronau, University of Louisville, dissertation committee member, thank you for your statistical guidance, and tutelage in creating tables in Word.

My gratitude is extended to staff at WKU and UofL: Kelly Ising, UofL; Carolyn Hunt, WKU; Jackie Powell, UofL, Courtney Kerr, UofL. Elisabeth Knight, WKU. And, special thanks are offered to Jackie Foster, branch office administrator. 


\begin{abstract}
LEADERSHIP, CURRICULUM, INSTRUCTION, AND ACCOUNTABILITY SCORES; EVIDENCE FROM KENTUCKY SCHOLASTIC AUDITS
\end{abstract}

\author{
Rebecca Curry Todd
}

December 10, 2010

In 1983 the National Commission on Excellence in Education released A Nation at Risk, which triggered an extended era of school reform culminating in today's accountability movement. In Kentucky the school improvement process, in which principals play an integral part, is based on the Standards and Indicators for School Improvement (SISI--with nine standards and 88 indicators overall) and the Scholastic Audit, a measurement tool based thereon.

Murphy's (2004) mediated model of instructional leadership recognizes that complex school reform necessitates both direct and indirect skills. The central research question captures the purpose of this study, testing Murphy's model: Based on Scholastic Audits, what are the effects of instructional leadership on Kentucky accountability scores, as mediated by curriculum and instruction?

Secondary data from 83 high schools in Kentucky are examined to determine the direct and indirect effect of leadership on student achievement. Descriptive statistics were calculated for nine demographic factors and the composite Academic Index (AI), an accountability measure. Factors for Standard 7 (Leadership), Standard 1 (Curriculum), and 
Standard 3 (Instruction) were derived from exploratory factor analysis of the 11, 7, and 8 indicators, respectively, for the standards. Cronbach's alpha and inter-scale correlations were also calculated. Simultaneous multiple regressions examined relationships among demographic factors, three standards, and AI. The full mediated effects model was tested with hierarchical regression.

The three sets of indicators each produced a single factor solution explaining $45.4 \%, 52.9 \%$, and $48.2 \%$, respectively of the variance. Cronbach's alpha ranged from .837 to .873 . Standard 7 (Leadership) explained $39 \%, 40 \%$, and $20 \%$, respectively, of the variance on Curriculum, Instruction, and the AI. Standards 1 and 3 combined explained $40 \%$ of the AI. Demographic factors explained $65 \%$ of variance on AI; each of the three standards was essentially independent of demographic controls. In final hierarchical model, Leadership added .06 to the variance explained in Step 2. Step 3 with Curriculum and Instruction added produced an additional .05; all that influence came from Standard 3 (Instruction). The final model had an effect size of $76 \%$, establishing empirical support for Murphy's mediated effects model of instructional leadership for high schools in Kentucky. 


\section{TABLE OF CONTENTS}

PAGE





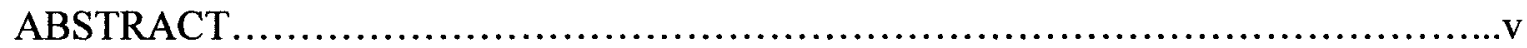

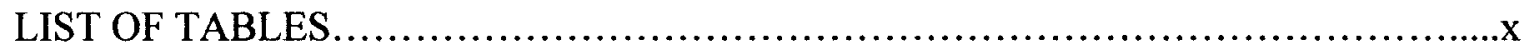

I. STATEMENT OF THE PROBLEM

Leadership Reform.............................................................. 1

Educational Leadership.................................................. 2

The Kentucky Education Reform Act .............................................4

Instructional Leadership in Kentucky.......................................

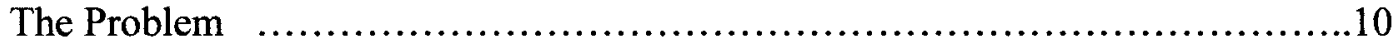

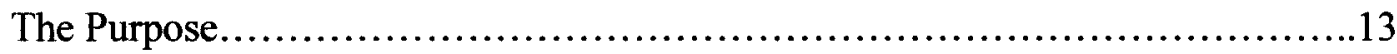

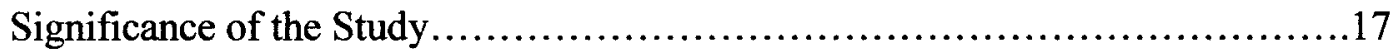

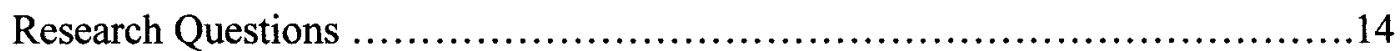

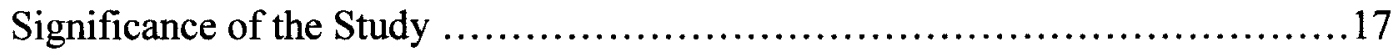

Limitations of the Study .....................................................

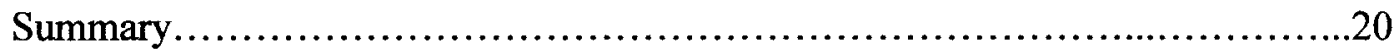

II. REVIEW OF THE LITERATURE








Leadership in an Era of Change...........................................28

Educational Leadership......................................................44

The Accountability and Standards Movement.................................56

The Kentucky Education Reform Act.....................................65

Accountability in Kentucky Education.....................................66

Standards and Indicators for School Improvement............................69

Developing the Standards and Indicators for School Improvement...............71

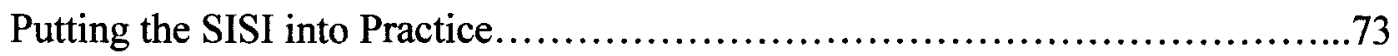

Curriculum, Instruction, and Achievement................................ 74

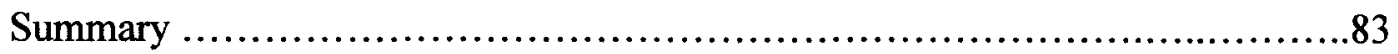

III. METHODOLOGY

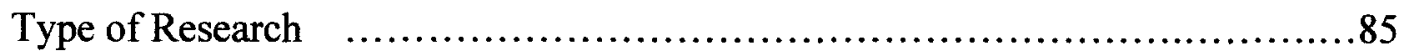

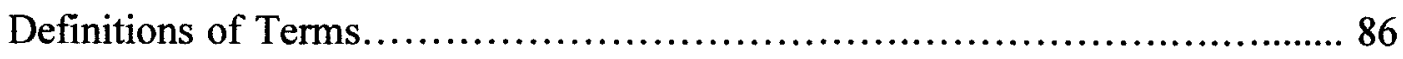

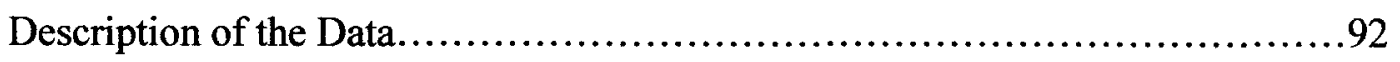

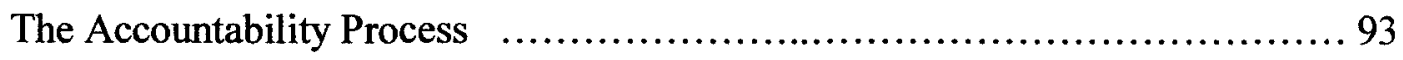

Scholastic Audit Methodology ................................................. 94

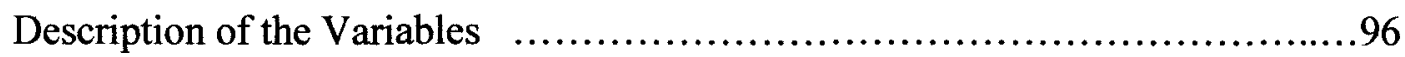

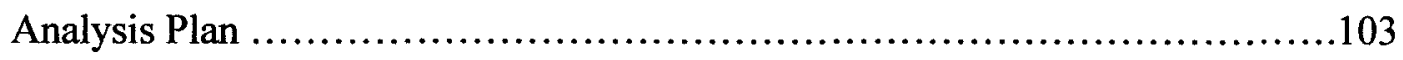

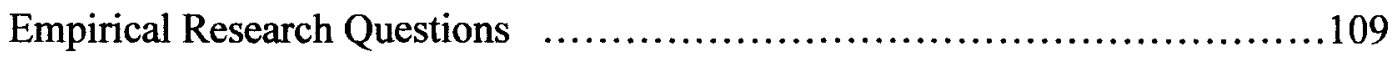

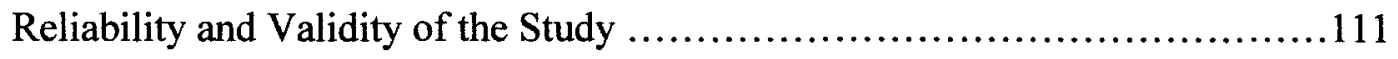

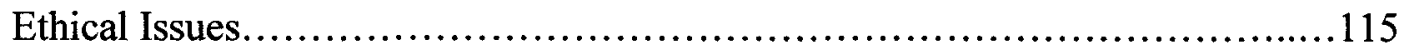

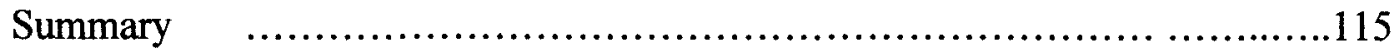

IV. RESULTS 
Introduction

Descriptive Statistics................................................... 120

Psychometric Analysis................................................... 123

Research Questions......................................................146



V. DISCUSSION AND CONCLUSIONS

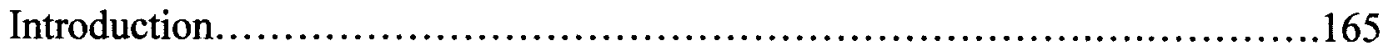

The Study in Brief....................................................... 168

Discussion............................................................... 171

Recommendations $\quad$................................................. 188

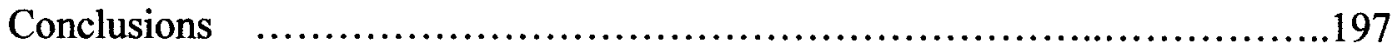



APPENDIX A.............................................................. 215

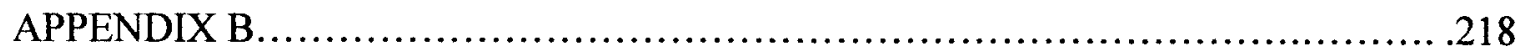

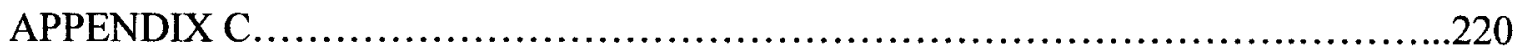





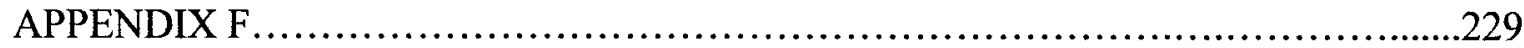

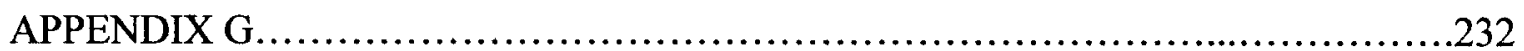

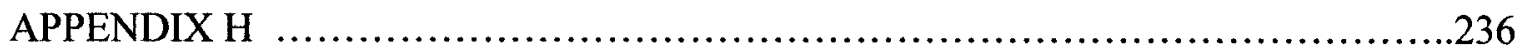

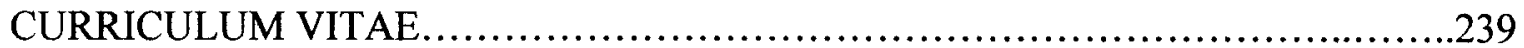




\section{LIST OF TABLES}

TABLE

PAGE

1. Variables and Types of Multiple Regression by Research Question.

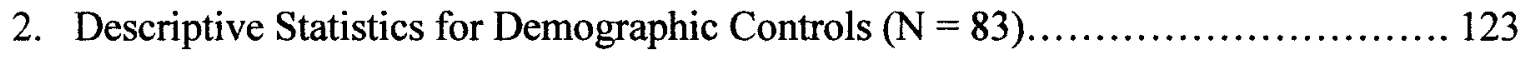

3. Total Variance Explained for Standard 7, Leadership..............................130

4. Factor Loadings for Standard 7, Leadership...................................132

5. Internal Reliability and Item Characteristics for Standard 7, Leadership

$(\mathrm{N}=83)$

6. Total Variance Explained for Standard 1, Curriculum 136

7. Factor Loadings for Standard 1, Curriculum. 138

8. Internal Reliability and Item Characteristics for Standard 1, Curriculum $(\mathrm{N}=83)$.

9. Total Variance Explained for Standard 3, Instruction

10. Factor Loadings for Standard 3, Instruction.

11. Internal Reliability and Item Characteristics for Standard 3, Instruction $(\mathrm{N}=83)$

12. Correlations for Demographics Factors, Leadership, Curriculum, Instruction, and Academic Index $(\mathrm{N}=83)$ 145

13. Regression of Standard 7, Leadership on Demographic Variables $(\mathrm{N}=83)$.

14. Regression of Standard 1, Curriculum on Demographic Variables $(\mathrm{N}=83)$. 150 
15. Regression of Standard 3, Instruction, on the Demographic Variables

$(\mathrm{N}=83)$

16. Regression of the Academic Index on Demographic Variables

$(\mathrm{N}=83)$

17. Regression of Standard 1, Curriculum, on Standard 7, Leadership

$(\mathrm{N}=83)$.

18. Regression of Standard 3, Instruction, on Standard 7, Leadership

$(\mathrm{N}=83)$.

19. Regression of the Academic Index, on Standard 7, Leadership

$(\mathrm{N}=83)$

20. Regression of the Academic Index on Standard 1, Curriculum, and Standard 3, Instruction $(\mathrm{N}=83)$

21. Regression of the Academic Index on Standard 7, Leadership, Controlling for Demographic Factors and as Mediated by Standard 1, Curriculum, and Standard 3, Instruction ( $\mathrm{N}=83$ )

Step 1

Step 2 160

Step 3 161 


\title{
CHAPTER I
}

\section{STATEMENT OF THE PROBLEM}

\author{
Leadership Reform
}

Recent scandals in corporate America as well as with political leadership have initiated large scale attrition of employee confidence in management over the last decades (Kouzes \& Posner, 1993, p. 25). In many organizations, dissatisfaction with leadership is extremely high (p. 33). Additionally, with expectations changing at an increasingly rapid rate, even leaders with long tenure appear to manifest incompetence (Leithwood, Begley, \& Cousins, 1992, p. 11). Educational leadership does not stand apart from American leadership. School leaders are steadily analyzed in the same venue of leadership and receive no less scrutiny of expectations (L. S. Ennis, 2002).

"No one can say for certain how the schools of the new century will differ from those of the past century--but there can be no doubt that these schools will require different forms of leadership (Hale \& Moorman, 2003). Schools share with other organizations the quest for leadership that makes a positive difference in their effectiveness and in the quality of life of the people who work in them (Storey \& Zellinsky, 1993). The role of educational leader requires meeting expectations from various and diverse interested parties such as teachers, students, parents, local leaders, and residents while functioning as a producer who sets goals and takes responsibilities for outcomes and as an integrator who builds professional relationships with staff and thus affects the culture of the workplace (Tornsen, 2009). 
Leaders in educational organizations face challenges from many sources. Dramatic and fundamental changes in economic and technological conditions are creating changes in social conditions at an unprecedented rate. The changing nature and patterns of employment, and the constant changes in technology, along with the workplace skills and competencies required by workers, are creating an even greater need for lifelong learners (Chapman, 1996).

\section{Educational Leadership}

Traditionally, schools have provided outcomes consistent with the labor needs of the American society (Cremin, 1961; Nasau, 1979; Perkinson, 1977; Tyack, 1974). Horace Mann, the first Massachusetts Commissioner of Education, said that "schools for all children would create a stable society in which people would obey the laws and add to the nation's political and economic well being" (Mann, cited in Hillway, 1964, p. 34). Knowledge was believed to be static as well as the complexity of schools, and the dynamics of achievement were consistent with the makeup of the student body (Meyer \& Rowan, 1977, 1978; L. S. Miller, 1992). Today, however, school leaders are confronted with a vastly different world on a daily basis and school expectations are tempered with current social and cultural paradigms; thus, the need for change.

The call for instructional leadership in the educational reform movement emerged in three distinct "waves" (Bjork, 1993, p. 247). The first wave began with A Nation at Risk (National Commission on Excellence in Education, 1983). The report drew attention to the need to improve achievement, mandate assessment, and increase accountability. State level regulatory requirements inhibited many leadership activities and reinforced top down management techniques. The second wave was defined by commission reports released by the Carnegie Forum on Education and the Economy, Task Force on Teaching as a 
Profession (1986), A Nation Prepared; the Holmes Group (1986), Tomorrow's Teachers; the National Governors Association (1986), Time for Results; and the Committee for Economic Development $(1985,1987)$ Investing in Our Children and Children in Need, respectively. These reports assumed that schools failed because of bureaucratic inefficiencies and recommended restructuring the education system. The third wave surfaced during 1988 , focusing on the need to lessen environmental factors responsible for children's lack of success in school and called for a redesigned structure for schooling. This wave proposed a child-focused restructuring that would provide an "integrated interorganizational service" system (Murphy, 1990, p. 29) including advocacy services for children and families as well as empower teachers, thus contributing to the need for better instructional leadership (Bjork).

In the current era of accountability, educational leaders need a clear understanding of what is expected and what is valued along with the personal and organizational resources to pursue valued goals. In response to this need, a consortium of state and professional associations, the Interstate School Leaders Licensure Consortium (ISLLC, 1996), published a comprehensive set of Standards for School Leaders. ISSLC (2008) subsequently published a revised set of standards. In response the Commonwealth of Kentucky also adopted, by statute, what is commonly called the Effective Instructional Leadership Act (EILA), KRS 156.101. The purpose of KRS 156.101 was to "encourage and require the development of effective instructional leadership" (EILA, 2006, p. 3) and assigned responsibility to the Kentucky Board of Education to "prescribe criteria for the training program" (EILA, p. 3). The KAR 3:325 identifies requirements of EILA including that "the content of programs approved for instructional leadership credit must consist of specific competencies which have applicability for improving the effectiveness of the 
instructional leader" (EILA, p. 3). Beyond the administrator standards, the Kentucky Department of Education (2004) developed nine Standards and Indicators for School Improvement (SISI), representing the application of the literature on school reform, school improvement, instructional effectiveness, leadership, and related areas (KDE, 2004c). The standards provide ultimate responsibilities of the educational leader in improving the academic performance of students, the learning environment, and organizational efficiency of his/her building.

\section{The Kentucky Education Reform Act}

The Commonwealth of Kentucky, in response to the prevailing call for change in schooling among the states, enacted the Kentucky Education Reform Act (KERA) in 1990. KERA was passed by state legislators as the result of a lawsuit filed by 66 Kentucky school districts. These districts challenged the state's funding formula which was deemed as relying too heavily on local resources, resulting in inequitable educational opportunities for the state's school children. Because of the lawsuit, the Kentucky Supreme Court ruled the state's educational system unconstitutional. The passage of KERA, with the aim to provide a means to improve education, was heralded as the most comprehensive education package ever passed by a state legislative body (Steffy, 1993) and essentially changed the face of education in Kentucky across finance, governance, and curriculum.

One drastic change was how governance was handled, including the introduction of school-based decision making (SBDM) councils in KRS 160.345. The school councils promote shared leadership among those who are close to the students. Membership of each council includes parents, teacher, and an administrator of the school (KDE, 2000). The council has the responsibility to set school policy and make decisions outlined in statute (KDE). David (1992), who studied early assessment of SBDM implementation, found that 
new councils met regularly, had organized into committees, and spent much time developing policy infrastructure (required council operating policies). Although most SBDM councils operate by consensus, the principal, as administrator of the school, chair of the council, and evaluator of teachers, exerts a major influence in determining how the council will operate (David, p. 4). Talley and Keedy (2006) established that councils may not begin to work as shared decision-making bodies until principals first "signal" by their actions within council deliberations their willingness to accede to other council members' expertise. Although principals may not see themselves as equals with their staffs, leadership can be distributed through the school as an organization (Ogawa \& Bossert, 1995 ) in part through the collegial efforts of the principal (Talley \& Keedy, p. 443).

To perpetuate the goals of increased student achievement, the Kentucky Department of Education (KDE), through KERA, established accountability guidelines for schools (Steffy, 1993) designed to be consistent with current theory, research, and experience (Fuhrman, 2001; Linn, 2000, 2003; Smith \& O'Day, 1991). KERA established ambitious performance standards. These new goals essentially constituted a value-added performance assessment model (L. S. Miller, 1992; Steffy); the architects of KERA believed these improved targets could reasonably be achieved, given sufficient effort and supporting resources (cf. Linn, 2003). These definitive accountability guidelines for schools place increased pressure on school leadership to utilize the knowledge base to advance curriculum and instruction.

The Kentucky goals address what leaders need to know and be able to do to support students and teachers in high performing schools (Kentucky Department of Education, 2006b). School leaders have a broad array of responsibilities, but none more important in Kentucky's high-stakes accountability arena than the implementation and monitoring of 
the goals and standards that clearly focus on instructional leadership. School leadership in Kentucky public schools, in accordance with the EILA Technical Assistance Manual, is obligated to support the following (KDE, p. 7):

1. Standards and Indicators for School Improvement (SISI)

2. Interstate School Leaders Licensure Consortium (ISLLC) Standards for School Leaders

3. Kentucky State Board of Education Goals and Objectives

4. Kentucky Department of Education Standards for Professional Development.

Additionally, the Effective Instructional Leadership Act (EILA) gives school

leaders instructional precedence as follows:

Legislative action, KRS 156.101, established legal support to encourage and require the maintenance and development of effective instructional leadership in the public schools of the Commonwealth and to recognize that principals, with the assistance of assistant principals, supervisors of instruction, guidance counselors, and directors of special education have the primary responsibility for instructional leadership in the schools to which they are assigned. (KDE, 2006b, p. 4)

A technical assistance manual, specifically for school leaders, clearly outlines instructional leadership duties as follows:

1. making instructional decisions that support teaching and learning;

2. establishing organizational direction;

3. developing and supporting high performance expectations;

4. creating a learning culture; and,

5. developing leadership capacity. (KDE, 2006b, p. 4)

With Kentucky principals bearing the liability for their school's success, they are central to the accountability consequences of their leadership. As felt nationwide, Kentucky principals are aware of this intense accountability pressure that is a driving force in the 
decisions and activities made for their schools (McKinney, 2007).

The Standards and Indicators for School Improvement (SISI) document was adopted by the Kentucky Board of Education as a set of guidelines regarding efforts to increase student achievement (KDE, 2004d). The document consists of 88 indicators spread across nine standards. This document represented a breakthrough in educational reform with Kentucky being the first state to extend the standards-based accountability movement based on content areas to areas of whole school reform. The nine standards represent different facets of overall school restructuring. Since Kentucky's development of the SISI, several states have adopted or adapted these standards. Associated documents-District Level Performance Descriptions and Glossary for Kentucky's Standards and Indicators for School Improvement (KDE, 2004a) and a supplementary document for schools, School Level Performance Descriptors for Kentucky's Standards and Indicators for School Improvement (KDE, 2004c)--identify school leaders' responsibilities in three specific areas: (a) Academic Performance, (b) Learning Environment, and (c) Organizational Efficiency. Decisions and actions of instructional leadership are anticipated to be in alignment with the competencies outlined in the SISI document.

These new concepts changed the expectations of principal leadership in Kentucky. Many new processes were open-ended; schools and school leadership had previously operated with more structured policy. This confusion raised many questions and fostered opposition in certain areas (McKinney, 2007). State leaders quickly realized that a new type of school administration would be needed to guide reform policy. It became imperative that mediated (the indirect influence of the principal on student success) leaders would have systems in place to know where students are concerning academic success, to track growth, and to take action when insufficient progress is being made (Murphy, 2004, 
p. 86). This dynamic of close monitoring of student performance and rapid response would become the new characterization for the principal under Kentucky's value-added accountability system, which effectively ended the traditional assumptions that schools are static organizations based on the "quality" of students, a euphemism for class and race (L. S. Miller, 1992). Instead, Kentucky now expected continuous upward movement from an initial baseline score and all students would be included in improvement measures indicative of curriculum and instruction decisions.

\section{Instructional Leadership in Kentucky}

With KERA came a philosophical shift in principal responsibility in the state. In recent years, much attention has been given to the fact that effective school principals are instructional leaders (Hallinger, Bickman, \& Davis, 1996; Hallinger \& Murphy, 1985). Instructional leadership, embossed within the state's legislative action, is a key requirement in Kentucky's school reform and is a significant factor in Kentucky audits of school leadership (KDE, 2006b). In leading schools, Kentucky principals have a vast assortment of responsibilities, with the primary focus on high-stakes accountability that revolves around increased student achievement. Directing school decisions that nurture student success makes it necessary for principals to create a culture that supports teaching and learning.

\section{Performance Assessment in Kentucky}

Knowing the effectiveness of principal leadership based on direct evidence would be useful in determining the relationship between instructional leadership and curriculum and instruction. The yardstick for such a measurement is Kentucky's system of performance assessment. When the Commonwealth of Kentucky enacted KERA, the KDE created an accountability measure, the Kentucky Instructional Results Information System 
(KIRIS), standardized testing administered to all students throughout the state each year, with limited exceptions. In 1998 KIRIS was replaced with a new but more traditional accountability system, the Commonwealth Accountability Testing System (CATS), to improve reliability. CATS combines criterion-referenced assessment results on the different content areas (e.g., math, science, English, etc.), along with norm-referenced scores and non-cognitive results (e.g., attendance, dropout, retention, and graduation rates) into a summary for all students of a school, resulting in an overall Accountability Index. The index value identifies a school's progress, or lack of, toward the goal of proficiency (Petrosko, 2000) and is given considerable importance toward the reputation of a school as well as being an indicator of effective school leadership over time (Lockwood, 1994).

Each school is expected to have an Accountability Index score of 100 by the year 2014, a very high standard (cf. Petrosko, 2000; Rothstein, 2004, ch 3). Within the overall Accountability Index, there is an Academic Index consisting of the statewide criterionreferenced tests in the different content areas, the Kentucky Core Content Tests (KCCT). Progress in meeting statewide goals is represented by the index scores for each school determined by a customized chart of the following categories: (a) meeting goal, (b) progressing, or (c) needing assistance.

The entire purpose of KERA is continuous improvement for students so that all schools reach Proficient on the accountability measures. To this end all schools in the needed assistance range were targeted for extra help and were required to engage in extensive school improvement, planning, and reforms. To facilitate these efforts, the KDE developed the Scholastic Audit, yet another Kentucky first in the field of accountability and reform (KDE, 2003).

The Scholastic Audit is based directly on the Standards and Indicators for School 
Improvement (SISI) and is a formalized mechanism for measuring each school's progress on meeting the standards expressed in the SISI. KDE developed 4-point behavioral descriptions for each of the $\mathbf{8 8}$ indicators. KDE-trained Scholastic Audit teams then conduct an intensive visit at each school, producing a consensus score for each indicator of each standard. Based on observations, interviews, and examination of school documents, the audit teams rate each of the 88 indicators of the standards of the SISI document. The audits provide a glimpse into how schools were progressing toward meeting the goal of proficiency by 2014, indicating how schools with a low Accountability Index differ from schools with a high Accountability Index. These records constitute a rich description of the inner workings of a school, both good and bad, and provide diagnostic evidence that can be utilized as the basis of focused, prescriptive school improvement efforts (McKinney, 2007).

All schools needing assistance were either required to receive a Scholastic Audit or to conduct an internal self-review. As indicated, the audits and reviews were based on the Standards and Indicators for School Improvement (KDE, 2003). The goal of the audits was to examine the three areas of the SISI document: Academic Performance, Learning Environment, and Efficiency. The teams trained by $\mathrm{KDE}$ consisted of a parent, teacher, school administrator, district administrator, university faculty member, and a Highly Skilled Educator.

The Problem

Kentucky has aligned its standards for leadership with the Interstate School Leaders Licensure Consortium (ISLLC, 2008) and the Standards and Indicators for School Improvement (KDE, 2003), as well as associated documents (KDE, 2004a, 2004c). Accordingly, Kentucky's leadership development efforts need to be concentrated in areas 
that will charter current and future instructional leaders to high levels of these adopted standards. This requires assessing the impact of school leaders on student success, consistent with these various philosophies and standards, assuming that they are meaningful measures.

Several strands of research are relevant to this broader problem, i.e., understanding the impact of school leaders on school improvement, particularly improved student performance. First, although there has been considerable work on the notion of mediated instructional leadership, there are few empirical tests of this model. Significant exceptions to this are the work of B. C. Ennis (2007), McKinney (2007), and Owings, Kaplan, and Nunnery (2005).

A second related strand is the work in Kentucky based on the relationships between the Standards and Indicators for School Improvement and school outcomes. The nine standards of the SISI document, each with its own set of research-based indicators, are reorganized into three sections: Academic Performance, Learning Environment, and Efficiency (KDE, 2003). Understanding the extent to which these three components affect school success would represent a significant step in interpreting instructional leadership effectiveness, particularly under the auspices of the value-added assumptions being tested (L. S. Miller, 1992). To date this approach has been tested empirically through a series of dissertations that have focused on SISI as measured by the Scholastic Audit--three completed at the elementary level (B. C. Ennis, 2007; McKinney, 2007; Saravia, 2008) with another in progress (Harvey). Regarding the work on Scholastic Audits, three specifically test a mediated model of school leadership: (a) McKinney; (b) B. C. Ennis; and (c) Harvey. This work replicates the study by McKinney except the current effort utilizes data from high schools. 
The work of McKinney (2007) is particularly germane. Much of what principals, as instructional leaders, do is concentrated on standards related to the areas of curriculum and instruction. The interplay among these three standards is at the hub of the pursuit of highstakes accountability goals in most schools. At the elementary level, McKinney investigates these relationships directly. As represented in the SISI (KDE, 2004d), these three standards are:

Standard 1: The school develops and implements a curriculum that is rigorous, intentional, and aligned to state and local standards. (p. 6)

Standard 3: The school's instructional program actively engages all students by using effective, varied, and research-based practices to improve student academic performance standards. (p. 8)

Standard 7: School/district instructional decisions focus on support for teaching and learning, organizational direction, high performance expectations, creating a learning culture, and developing leadership capacity. (p. 20)

A complete list of the nine standards appears in Appendix A.

The last strand is work investigating these issues at the high school level. To date, no work was located on the SISLScholastic Audit for high schools. Regarding mediated instructional leadership at the high school level, the author is unaware of any tests of this model. Thus, the dearth of empirical data on these three strands generally--on mediated instructional leadership, on the Standards and Indicators for School Improvement as portrayed via data collected with the Scholastic Audit; and for Kentucky principals at the high school level--represents the problem addressed in this study. Additionally, the data from Kentucky's Scholastic Audit reflect school improvement efforts as influenced by high-stakes accountability.

The three standards from the Standards and Indicators for School Improvement constitute this study's efforts to determine the effect of leadership, more specifically, 
instructional leadership, on Kentucky performance outcomes as schools strive to achieve educational goals in a high-stakes atmosphere. Standard 7 definitively evaluates school leadership and acknowledges the focus of leadership largely via the school principal. Principals are legally identified (KDE, 2006b, p. 4) as the primary responsible party in providing instructional leadership in Kentucky schools. Standards 1 and 3 are designed to advance quality curriculum and instruction, respectively.

\section{Purpose}

Summarizing the previous sections, little systemic research has been conducted on the effects of leadership on school success in curriculum and instruction at the high school level. Although a professional consensus has developed suggesting that some characteristics of leadership make it more effective than other characteristics, there has been little direct evidence on how these characteristics relate to improved teaching and increased student achievement. Due to this lack of empirical evidence, the purpose of this study was to analyze the secondary data collected by the Kentucky Department of Education (KDE) through Scholastic Audits: (a) to examine the effects of school level leadership on curriculum and instruction as they pertain to student success depicted by the Academic Index scores in Kentucky high schools, and (b) to investigate relationships, if any, that may exist among certain demographic factors such as school size, school setting, gender, and student achievement.

The study specifically considers the effects of school reform in the Commonwealth of Kentucky. The Kentucky Education Reform Act from 1990 is widely recognized as the most comprehensive of all statewide reform efforts (Pankratz\& Petrosko, 2000). Although KERA shifted the state's policy emphasis to improving school outcomes, Kentucky policy initiatives also included extensive governance and finance reforms. In fact, the original 
lawsuit that led to KERA was a traditional finance equity suit (Steffy, 1993). However, the current study does not address initiatives in either the realm of governance (e.g., nepotism or SBDM--cf. Talley \& Keedy, 2009) or finance (e.g. equalization of funding formula--cf. Haselton, 2004).

Thus this research is limited to accountability-related reforms. Accountability, typically, is determined by standards-based performance measures, but these standards most often represent indicators in disciplines such as science, mathematics, or reading (cf. Clune, 1998; Ravitch, 1995). In contrast to most school reform movements, Kentucky was the first state to develop standards focused on school improvement. The KDE developed nine standards, each with subsequent indicators. The standards are divided into three categories, each with three standards. The Standards and Indicators for School Improvement (SISI) represents the application of the literature on school reform, school improvement, instructional effectiveness (Hallinger \& Murphy, 1986), leadership, and related areas and is the basis of all Scholastic Audits and Reviews.

The information for this proposed study represents a secondary data base obtained from KDE. Included are Scholastic Audits conducted by the KDE audit teams on selected high schools in Kentucky. Data are supplemented by information obtained by KDE on school demographics and Academic Index outcomes. The investigation will focus on three standards--Standard 1 (Curriculum), Standard 3 (Instruction), and Standard 7 (Leadership). Thus, the central research question for this study is: Based on Scholastic Audits, what are the effects of instructional leadership on Kentucky accountability scores, as mediated by curriculum and instruction?

\section{Research Questions}

The purpose of this study is: (a) to examine the effects of school level leadership on 
curriculum and instruction as they pertain to student success depicted by the Academic Index scores in Kentucky high schools, and (b) to investigate relationships, if any, that may exist among certain demographic factors such as school size, school setting, gender, and student achievement. The data came from Kentucky's Scholastic Audits for school improvements. Figure 1 represents a model of the theoretical relationships among these variables, controlling for effects of demographic influences. Demographic factors to be tested are derived from the previous work of B. C. Ennis (2007), McKinney (2007), and Saravia (2008).

Specific empirical research questions for this study are derived from Figure 1, as follows:

1. To what degree do demographic factors affect Standard 7 (Leadership), Standard 1 (Curriculum), Standard 3 (Instruction), and the Academic Index in Kentucky high schools?

2. To what degree does Standard 7 (Leadership) affect Standard 1 (Curriculum), Standard 3 (Instruction), and the Academic Index in Kentucky high schools?

3. To what degree do Standard 1 (Curriculum) and Standard 3 (Instruction) affect the Academic Index in Kentucky high schools?

4. To what degree do Standard 1 (Curriculum) and Standard 3 (Instruction)mediate the effect of Standard 7 (Leadership) on the Academic Index, controlling for demographics in Kentucky high schools? 

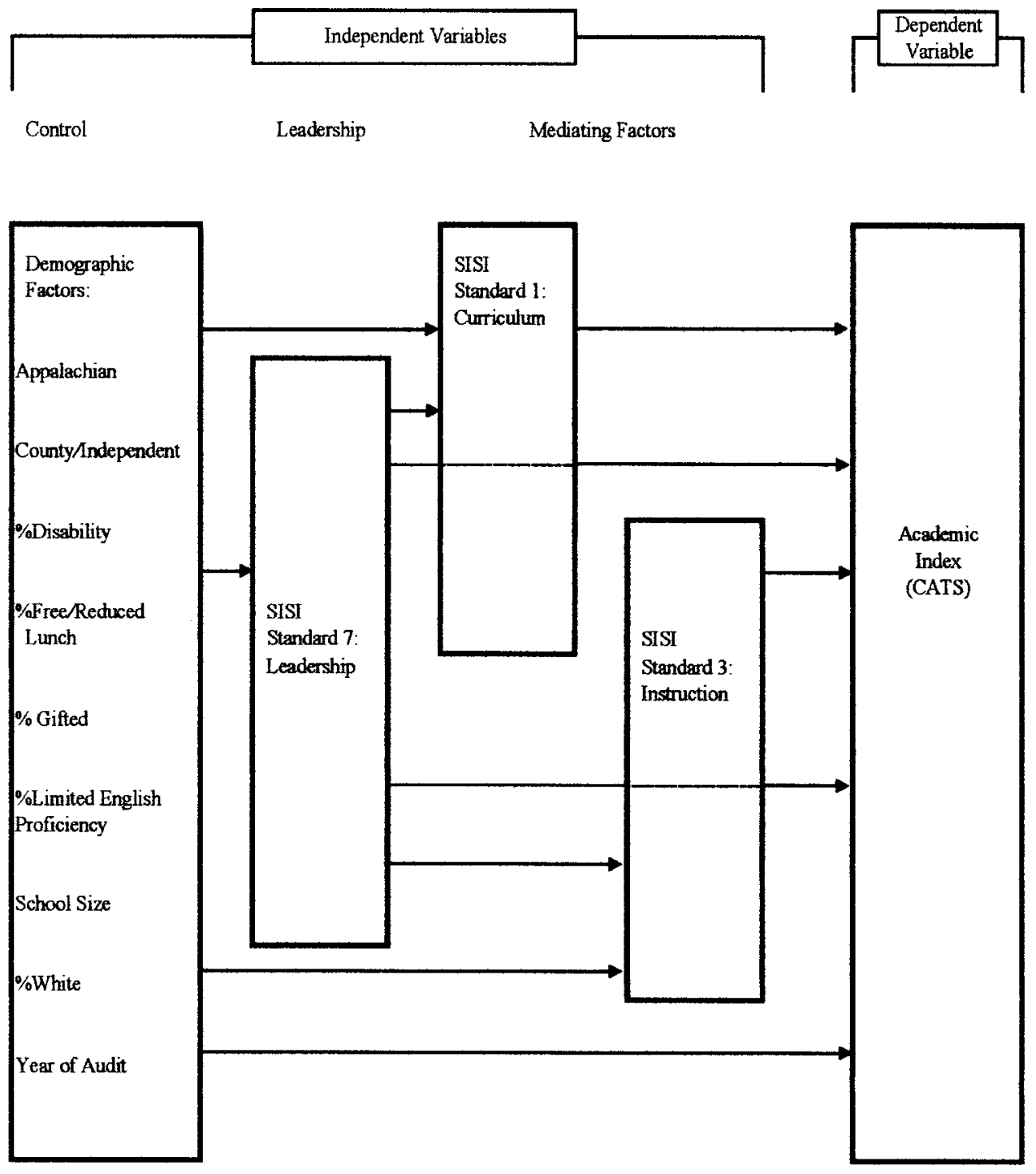

Figure 1. Hypothesized relationships for a mediated effects theoretical framework of instructional leadership and achievement. 


\section{Significance of the Study}

The current study examines mediated leadership, the indirect influence of the principal on student success through the efforts in curriculum and instruction. Although mediated leadership is widely acknowledged in the literature (see Murphy, 2004), the relative absence of empirical research in this area makes this investigation significant. Specific contributions follow.

First, the current study provides the opportunity to examine instructional leadership in conjunction to curriculum and instruction in the context of arguably the most comprehensive package of reforms among the 50 states (Pankratz \& Petrosko, 2000), including high-stakes, value-added accountability testing (L. S. Miller, 1992). The Kentucky Education Reform Act of 1990 has produced several statewide innovations in the current era of accountability.

Second, Kentucky was the first state to expand the assumptions of standards-based curriculum reform to whole school reform (SISI). These standards provide a framework for understanding and conducting school improvement. Although other states have adopted or adopted the SISI, this perspective provides a context unique from most states.

Third, the KDE developed a school evaluation process based on the standards of the Scholastic Audit, utilizing trained external teams composed of a parent, teacher, school administrator, district administrator, university faculty member, and a Highly Skilled Educator. The audits assess each indicator of the nine standards, thus compiling a profile of the school's practices in curriculum and instruction (Academic Performance); attitudes, beliefs, and values related to learning (Learning Environment); and the structures of organization, planning and coordination, and leadership (Efficiency). Kentucky has the most extensive Scholastic Audit data set in the nation. 
Fourth, little research on mediated leadership at the high school level is available in the academic literature. Thus, the KDE secondary data base on Kentucky high schools will provide new insights characterizing leadership as it pertains to curriculum and instruction, i.e., high schools are much larger and more complex than are elementary organizations.

Fifth, previous studies based on Scholastic Audit data in Kentucky (Ennis, 2007; McKinney, 2007; Saravia, 2008) have been at the elementary level. Ongoing research on the Scholastic Audit in Kentucky is at the elementary level (Harvey) and middle school. This is the only study to date of Kentucky Scholastic Audit data at the high school level.

Sixth, this study represents a partial replication of the McKinney (2007) study except at the high school level rather than the elementary level. There are simply too few replications in educational research, a notable strength of this work.

\section{Limitations of the Study}

The purpose of this study is: (a) to examine the effects of school level leadership on curriculum and instruction as they pertain to student success depicted by the Academic Index scores in Kentucky high schools, and (b) to investigate relationships, if any, that may exist among certain demographic factors such as school size, school setting, gender, and student achievement. The data came from Kentucky's Scholastic Audits for school improvements. Because this investigation is specific to location, variables examined, and the characteristics of the secondary data base, there are several limitations. Specifically, leadership is an evolution of circumstances particular to a specific time (Hallinger \& Heck, 1998, p. 159); therefore, researchers must contend with school leadership characteristics as an anti-static phenomenon. Other limitations are innate to the study of instructional leadership that embodies context, content, and methodological issues. Specific enumerations follow. 
First, the underlying document, Standards and Indicators for School Improvement:

Kentucky's Model for Whole School Improvement (KDE, 2003) was originally developed in Kentucky. Although other states have since adopted or adapted some version of the SISI document, generalization to other states at this point is limited. Even those states that have begun to utilize SISI do not yet have a database as extensive as that accrued in Kentucky.

Second, policies and accountability testing are not identical from one state to the next. Thus, the data and subsequent study are confined to Kentucky as well as the results obtained under the KERA reforms may not be generalizable to other states.

Third, the results depend on the Kentucky Core Content Tests in areas such as math, science, English, etc., which constitute the Academic Index (the dependent variable for this study) so that results may not be comparable to other states.

Fourth, only high schools are analyzed in this study. Elementary and middle school settings can be considerably different, which limits generalizability to all school levels.

Fifth, the data were collected by different audit and review teams. Although the teams are trained by KDE (2003) and all teams use the same documents to gather data, there is no psychometric analysis of the reliability of the different teams. Koger and Thacker (2004) did, however, conduct a preliminary study of the validity of the audit process. They stated "while there were no reliability measures taken to assess inter-rater reliability, all teams did use a standardized instrument and received training on conducting the audits/reviews" (p. i).

Sixth, because all audits were not conducted within the same school year, changes may have occurred within the schools based on the previous year's accountability score. The matrices of the schools may have changed from year to year as students, various teachers, and principals may have changed during the 2-year cycle of the audits. 
Consequently, the audits were attached to the Academic Index for the year in which it was conducted so that conditions measured at least corresponded to the activities at the time the audits were conducted.

Lastly, the reviews occurred during a time of federal (No Child Left Behind Act 2001) and state regulations that may have changed the degree to which efforts toward student achievement caused perspectives and activities to vary but are not accountable in the data for this study. As with any study also based on standards and political mandates, not all such provisions are supported by research.

\section{Summary}

Since the National Commission on Excellence in Education (1983) report titled $A$ Nation at Risk criticized public education in America, education has been in various stages of reform. The Commonwealth of Kentucky answered the call for reform by enacting the Kentucky Education Reform Act in 1990. This reform, which has redefined roles and responsibilities of shareholders, specifically placing new emphasis on the instructional leadership or principal position, has been acknowledged across the nation as a frontrunner of reform (Pankratz \& Petrosko, 2000).

An aspect of Kentucky's reform act is the Commonwealth Accountability Testing System (CATS). This value-added assessment instrument is administered to school children across the state each year with the purpose of monitoring student achievement in every school and district. Kentucky schools are required to make steady progress toward the goal of proficiency (a score of 100) by 2014 (Petrosko, 2000).

To promulgate school progress, the Kentucky Department of Education (KDE, 2003) adopted a series of standards, the Standards and Indicators for School Improvement: Kentucky's Model for Whole School Improvement (SISI), which includes the most relevant 
aspects for educators to recognize as key to improving student achievement. Depending on the progress of a school toward its goal of state-mandated proficiency, schools can be identified as in need of "assistance." The General Assembly passed additional legislation that required the Kentucky Board of Education to adopt administrative regulations to establish consequences for these schools. One result was the establishment of the Scholastic Audits based on the SISI document. This document, a set of nine standards and their respective indicators, measures school structures, basic processes related to curriculum and instruction, supportive activities, and attitudes and values related to performance outcomes (KDE, 2004c).

The KDE assembled and trained teams to conduct Scholastic Audits during the 2000-2001, 2002-2003, and 2004-2005 school years. Based on preliminary analysis of these data, the KDE (2003) identified Leverage Points (see Appendix C) and Variance Points (see Appendices D, E, and F) which distinguished successful schools from "needs assistance" schools. The KDE commissioned one study (Koger \& Thacker, 2004) to conduct initial analyses of the Scholastic Audits but other formal studies have not been conducted by the state.

With the current reform in Kentucky, the weight of responsibility for school success or failure has been shifted from district personnel to building level leaders, the school principals. Because the accountability of school success is so demanding, many school principals need information on what works and which strategic efforts provide the greatest return for invested effort. To date, there are limited if any studies available which compare results of the Scholastic Audits for high schools in Kentucky. Nor have there been any efforts to examine these data with respect to demographic background or theoretical linkages among the nine standards. 
Specifically, at present there are three dissertations that investigated the impact of the Scholastic Audit and achievement outcomes for specific standards in Kentucky: McKinney (2007) on Leadership, Curriculum, and Instruction; Saravia (2008) on School Culture and Parent Involvement; and B. C. Ennis (2007) on Leadership and Professional Growth, Development and Evaluation. All these investigations were at the elementary level. Three other studies of the Scholastic Audit are in progress--two at the elementary level and one for middle schools. To date, no work on the Scholastic Audit has been conducted at the high school level. This study replicates McKinney (2007), but at the high school level: Standard 1 (Curriculum) and Standard 3 (Instruction) are examined for the extent that they mediate the effects of Standard 7 (Leadership) on student achievement, controlling for demographic background. Figure 1 illustrates the theoretical linkages. Consequently, this work addresses mediated instructional leadership at the high school level. The analysis is a quantitative analysis of secondary data from the Kentucky Department of Education. Although the Division of School Improvement audits contain information on all nine standards, for this study the nine standards represent too broad a scope. More specific is the central research question for this study: Based on Scholastic Audits, what are the effects of instructional leadership on Kentucky accountability scores, as mediated by curriculum and instruction?

Because of the technical nature of terms associated with accountability, assessment, and empirical measurements used in this research, a Definition of Terms section is required. These definitions are included in Chapter III. 


\section{CHAPTER II}

\section{REVIEW OF THE LITERATURE}

\section{Introduction}

The educational reform movement originating after the release of $A$ Nation at Risk (National Commission on Excellence in Education, 1983) has caused government and business leaders to increase pressure on schools across the United States to raise academic standards for all children. Mandates from federal and state levels challenge schools and school leadership toward the goal of higher academic achievement for all students with passage of value-added accountability systems, such as the Kentucky Educational Reform Act (KERA) of 1990 and the No Child Left Behind (NCLB) national legislation of 2001.

The national legislation turned up the pressure, especially for school leaders as its mandates brought with them the full weight of federal policy. The NCLB act required that schools bring all students--including students with special needs such as disabilities, English as a second language, ethnic minorities--to an adequate level of progress based on consensus achievements of typical students (Lashway, 2002, p. 2). The federal government rendered these mandates to the states' educational venues with an influx of approximately 6-10 percent of school budgets coming from federal aid (Schlechty, 2001, p. 223).

The reform initiative in Kentucky (KERA, 1990), is heralded as one of the most ambitious reforms ever undertaken at the state level. The extensive statewide changes involved policies designed to make governance more transparent and less political; equalize statewide funding formulas to increase equity, particularly for property-poor 
school districts; and require all schools to improve academically over time. Kentucky has committed to reach "proficiency" by the year 2014 meaning all schools will be at the Proficient level, 100 on the 140 point Academic Index scale of achievement. To assist schools in moving toward that goal, the Kentucky Department of Education (KDE) has developed two innovations both unique in the U.S. at the time, although several states have since adopted or adopted versions for their use.

The Standards and Indicators for School Improvement (SISI) (KDE, 2003) represented an extension of standards-based accountability from content areas to whole school reform: nine standards and $\mathbf{8 8}$ indicators divided into three areas: Academic Performance, Learning Environment, and Efficiency. Among these standards are Leadership (Standard 7, with a focus on instructional improvement), Curriculum (Standard 1), and Instruction (Standard 3), the three standards of particular emphasis for this study. Linking these is the leadership of principals, a key to schools achieving proficiency levels. Kentucky's expectations for school level leadership are examined in the context of school accountability, based on Scholastic Audits that record levels of implementation of the SISI. This allows an examination of how these standards affect school level achievement, the Academic Index.

Kentucky's high-stakes accountability measures progress of schools toward attaining proficiency by 2014 . For those schools not meeting their biennial achievement goals, the KDE requires through KRS.6455 that a Scholastic Audit be conducted. The Scholastic Audit uses the SISI to document a school's preparedness for increased academic achievement (KDE, 2005e). Each of the 88 indicators across the nine standards is scored on a 4-point behaviorally-defined scale. The audit is required for schools identified as not meeting their biennial goals; however, all Kentucky schools and/or districts can opt for a 
Scholastic Audit to provide diagnostic information as schools work toward meeting their goals.

Considering the emphasis placed on educational leadership in the current period of accountability, Kentucky has adopted standards for administrators (ISSLC, EILA). Although these standards provide a more definitive tone to professional expectations, resources are at times limited for proper implementation of the organizational and personal valued goals. This is clearly the case in regards to achieving goals at schools with large populations of at-risk and special needs students. Although Kentucky schools have made significant overall progress under KERA (the excellence dimension), there is strong evidence that improvements in closing achievement gaps (the equity dimension) have lagged behind (cf. S. K. Miller \& Moore, 2006).

It is beyond the scope of this paper to address all the components of KERA; this literature review focuses on the tenets of instructional leadership in Kentucky, particularly its connectivity to curriculum and instruction at the high school level. The remainder of this chapter reviews literature on Leadership, Educational Leadership, The Accountability and Standards Movements, The Kentucky Education Reform Act, and Standards and Indicators for School Improvement. The chapter ends with a Summary.

$$
\text { Leadership }
$$

Relatively new to the English language, the word leadership has only been in use for about 200 years although the term leader from which it was derived appeared as early as 1300 A.D. (Yukl, 1989, p. 3). Yukl categorizes leadership into four approaches: powerinfluence, behavior, trait, and situational. Leadership, as defined by Hersey and Blanchard (1982) is the "process of influencing the activities of an individual or a group in efforts toward goal achievement in a given situation" (p. 83). They also believed that leadership 
involved the components of leader, follower, and situational variables. On the other hand Hodgkinson (1991) suggests that "leadership is a mere incantation for the bewitchment of the led" (p. 53). He goes on to state that leadership and administration are interchangeable. Pearman (1998) states that "Leadership is in flux today, and the old paradigms for managing effectively are no longer enough. The key to success for millennium leaders is the ability to communicate meaningful information and build relationships among organizational members" (p. 21). This section reviews leadership starting with an examination of leadership styles.

In his analysis, Howard (2002) designed a study to facilitate awareness and provide instruction for leaders struggling with identification of their own leadership styles, as well as identification of the most appropriate styles of subordinates. According to Howard, the key questions to be answered when defining personal leadership styles are: (a) What is leadership? (b) What is your preferred leadership style? (c) How does our style impact the people, tasks, and environment that you are leading? (d) What is the preferred leadership style of the members of your leadership team? (e) What tasks are best assigned to team members based upon their preferred leadership style of your leadership team members?

The sample of Howard's 2002 study encased clients and colleagues representing more than 100 schools, colleges, and universities over a span of more than 30 years. Methodology for data collection was a comparative analysis of case studies and research completed at the University of Alabama, Harvard University, Fort Valley State University, Talladega College, Atlanta Metropolitan College, Miles College, Stetson University, The Citadel University, Webster University, and numerous school districts and technical colleges. Additional data influencing the research was a qualitative case study of the cognitive framework for the styles of leadership based on brain quadrant research by Ned 
Herrman who mentored the author at the University of North Caroling during the 1980s.

The findings of the Howard (2002) study indicated that all leaders of effective groups have four characteristics in common: (a) they provide direction and meaning to the people they are leading; (b) they generate trust; (c) they prefer action and risk-taking; and, (d) they are communicators of hope. Howard outlined four types of leadership styles: (a) Type-A (Fact Based): leaders who are comfortable with people, tasks, and environments that require facts, logic, theories, scientific applications, analysis, quantitative, mathematical, and technical processes. They establish high quantitative standards of behavior and expectations for employees and themselves. They rarely show emotion; (b) Type-B (Creativity): leaders who prefer problem-solving techniques that involve artistic, flexible, imaginative, spontaneous, and holistic responses. They are open and direct in communicating with others. Their thinking and behavior reflect a creative, casual, and relaxed style; (c) Type-C (Feelings): leaders that prefer deciding how he/she feels about the people, tasks, and environment, often ignoring research and facts that are contrary to his/her decisions. They may prefer to use emotions, empathy, intuition, and interpersonal relationships to guide decisions. They are uncomfortable with data as a source for making decisions, unless the data are compatible with their feelings. They do not use the principles of science to analyze and solve problems, instead relying on intuition; (d) Type-D (Control/Power Based) leaders: use power and control over people, tasks, and environment. They lack imagination and creativity. They often expect all subordinates to play a submissive role to them, and they provide rewards accordingly. They choose control, details, planning, sequencing, and strict organization to respond to people, tasks, and environment.

In his study, Howard (2002) determined that leaders develop perceptions, attitudes, 
and values for the stimuli in the world around them based upon their genetic predispositions and the results of the environmental impact during their informative years. Therefore, potential leaders may prefer a particular way of processing stimuli and making decisions. He surmised that making a key step toward successful leadership is having the range of skills that allows usage of appropriate leadership skills according to the situation and assign tasks to leaders based on their preferred leadership style. However, he also canted that leadership styles can be learned. He suggested that a long-term staff development system should be implemented to assist leaders in developing a holistic leadership style that encompasses the ability to use all four styles based upon the situation.

\section{Leadership in an Era of Change}

Leaders in educational organizations face challenges from many sources. Dramatic and fundamental changes in economic and technological conditions are creating changes in social conditions at an unprecedented rate. The changing nature and patterns of employment, and the constant changes in technology, along with the workplace skills and competencies required by workers, are creating an even greater need for lifelong learners (Chapman 1996) and innovative leaders. "The 'good old days' are long gone when an educator's best judgment constituted sufficient proof of learning outcomes" (Lezotte $\&$ Bancroft, 1985, p. 25).

Fennell (2005) purposed that education is at the forefront of changes in society, with personnel at all levels of the educational structure on the "front lines" (p. 145). Restricted not just to the classroom, public scrutiny ranges from finance to curriculum, creating challenges for educators (principals and teachers) to "develop new ways of working in schools" (p. 145). Leadership may be in different forms. Viewing leadership from different philosophies changes the ways it is perceived. What leadership looks like 
from one perspective may not be what it looks like from another.

\section{Leadership in Professional Communities}

Historically, schools in North America emerged in farming communities established to meet the educational and societal needs of the communities. Thus, as Beck and Foster (1999) noted, the concept of school as learning communities is traced back to a time when "[s]chools typically supported local values and manifested relational patterns that were congruent with surrounding neighborhoods. Such schools were assumed to be both reflections and extensions of their communities"(p. 146).

Professional learning is closely associated with schools as communities. The educational arena can be a lonely profession, often lacking in interaction among peers (Lieberman \& Miller, as cited by Fennell, 2005). Yet as Barth (1990) noted, in professional communities it is important that the learning of teachers and school leaders be emphasized to the same degree as that of students. Recent research on schools as communities also emphasized systems of shared, commonly understood values among teachers, leaders, and students (Fennell, p. 132). Mitchell and Sackney (as cited by Fennell, p. 132), offered that the sharing of common values, beliefs, understandings and goals "serve as a glue that holds members together....And, in a professional community, everyone is an important and worthwhile participant and shares in the tasks, activities, and responsibilities of the community, and that each person deserves the support and care of other community members."

Collaborative communities present the constant challenge of ensuring that everyone takes some part in the power base and shares in making decisions that affect their participation and work in the community (Fennell, 2005). Grogan (as cited by Fennell) stressed that "the discourses in which we participate teach us what to do and how to do 
things approved by the discourses and how to avoid what is proscribed...that dissent among community members is an essential part of discourse and that democracy is not well served by suppressing contrary dialogues" (p. 127). Discourse, then, among community members is essential in developing knowledge and power. By giving voice to many versions of social reality, discourse and decision-making are likely to be more inclusive and better informed. Senge's (1990) vision described the new work of leaders in such organizations as responsible for learning that takes place within them:

In a learning organization, leaders' roles differ dramatically from that of the charismatic decision maker. Leaders are designers, teachers and stewards. These roles require new skills: the ability to build a shared vision, to bring to the surface and challenge prevailing mental models, and to foster more systemic patterns of thinking. (p. 127)

Lambert (1998), an advocate of constructivism, described leadership as, "The reciprocal processes that enable participants in an educational community to construct meanings that lead toward a common purpose of schooling" (p. 33). She elaborated that leadership is not finite and not restricted by formal authority and power; that it instead permeates a healthy school culture and is undertaken by whoever sees a need or an opportunity. She defined the four characteristics of reciprocal relationships: (a) evoke the potential in a trusting environment fostered by positive relationships, (b) reconstruct the environment by 'breaking set' with old assumptions and myths, (c) set a clear focus on the construction of meanings, and (d) frame actions that embody new behaviors and purposeful intentions (p. 33).

Hipp (2001) conducted a case study to examine issues of trust, specifically the effects of mistrust on risk, relationships, organizational health, and openness to change while trying to create a professional learning community. The study was framed on a review of literature by Hord (1997) which was one of five case studies that were part of a 
national project called Creating Continuous Communities of Inquiry and Improvement. In the study, Hipp cited five dimensions of successful professional learning communities: (a) shared and supportive leadership; (b) shared vision and values; (c) collective learning and application; (d) supportive conditions; and (e) shared personal practice.

The study concentrated on fifty-one professional staff members--30 female and 21 male--in a Midwest school in a middle-income suburban district. The teachers were predominantly European American with 37 teachers having more than 15 years of experience and 82 percent with a master's degree. The student body of 550 was $12 \%$ free and reduced lunch. To collect data, the researcher used multiple methods: (a) telephone interviews; (b) face-to-face interviews ( $25 \%$ of the staff was interviewed on-site); and (c) a questionnaire administered three times over a three-year period. All interviews were audiotaped, transcribed and analyzed by a six-member team, according to Hord's five dimensions (as cited by Hipp, 2001). The findings from the study included: (a) unattended feelings fester and grow; (b) voice cannot be ignored; (c) mistrust affects relationships, organizational health, and openness to change; and (d) perceptions of favoritism are detrimental to change. Educators can draw significance from the study because of its "reallife" interpretation that can be transferred to actual collaborative group settings to spawn inquiry and solutions.

An exploratory study was conducted by Cowley and Meehan (2003) to investigate school improvement through professional learning communities and the professional staff's commitment to continuous learning and improvement in schools with high academic index scores. Research used for the study was grounded in previous work by Louis, Marks, and Kruse and by Hord (as cited by Cowley \& Meehan). Forty-eight high-performing schools (24 elementary schools, 12 middle, and 12 high schools) in the Commonwealth of 
Kentucky were identified for participation. Criteria utilized by the Kentucky Department of Education for school participant selection were: (a) test scores; (b) high percentage of free and reduced lunch student populations; (c) percentage of students in programs for struggling learners, such as Extended School Services; and (d) the percentage of African American student population. For further selectivity, the schools were placed in two groups: (a) students in the criterion categories were scoring within 10 points of the school average, and (b) students in the criterion categories were scoring 10 points below the school average.

The survey instrument, Appalachian Educational Laboratory (AEL) Continuous School Improvement Questionnaire, was used for the data collection process. The instrument, a 60-item self-reporting inventory, consisted of six key concepts: (a) shared leadership; (b) effective teaching; (c) school, family, and community connections; (d) purposeful student assessment; (e) shared goals for learning; and (f) learning culture. The survey used Likert-type responses $(1=$ not present to $6=$ present to a high degree $)$. Of the 48 schools selected, 47 completed and returned the survey for $98 \%$ participation. The Remark scanning software was used for data input and then exported to SPSS for statistical analysis. Wide variations in professional staff commitment to continuous learning and improvement between achievement gap groups and across building levels were indicated in the findings. Family and community connections posed the greatest need for intervention especially at the high school level. As would be anticipated, professional staff in the schools with higher achieving students held higher perceptions of their schools than those in schools with the less successful student population subgroups. In the schools with larger achievement gaps, learning culture, shared goals for learning, and effective teaching were areas of concern. 
Because of the findings, the researchers, Cowley and Meehan, suggested that for schools to address the successful academic achievement of all students, not only should faculty focus on structural changes, new standards, or accountability requirements, but also the focus should be on school climate to sustain teacher commitment to continual learning and improvement. The researchers cautioned generalizing this study to larger groups because of the small number of participants and suggested for future research that additional multi-level analyses should be applied to data.

Rafoth and Foriska (2006) conducted an evaluative study to determine the impact of administrator participation on problem-solving collaborative teams in light of enhanced student outcomes. The types of collaborative teams included pre-referral, teacher assistance, mainstream assistance, intervention assistance, and instructional support. From literature on qualitative studies the researchers concluded that effective principals involved key teacher leaders in shared decision making to a greater extent than did principals who were less effective.

In a qualitative study, Anderson (2004) interviewed 28 respondents from six Canadian schools identified as having high levels of teacher leadership. Additionally, these schools were undergoing federal mandates to develop school improvement plans. From his study, Anderson proposed three models of leadership reciprocity: (a) the buffered principal; (b) the interactive model; and (c) the contested model. Overall, he found that administrative and teacher leadership may experience contextual differences due to evaluation; however, the use of collaborative problem-solving teams may provide the mechanism for administrators to provide systematic ways to identify the needs of teachers and to deliver appropriate support.

Portin (2004) studied all grade levels in 21 schools in four states to examine how 
principals performed seven leadership functions: (a) instructional; (b) cultural; (c) managerial; (d) human resources; (e) strategic; (f) external development; and (g) micropolitical, in light of several variables that affect day-to-day responsibilities. Variables included: (a) time constraints; (b) external pressures; (c) direct involvement in discipline; and (d) demand for academic leadership. The findings indicated that collaborative problemsolving teams are a means for principals to capitalize on sharing instructional leadership responsibilities through the development of teacher instructional leaders. Portin synthesized a model of the impact of administrator support on collaborative problem-solving teams--the Interactive Principal-Teacher Influence Model. Portin indicated that the relationship between principal and staff--characteristics such as culture, student expectations, and teacher professionalism as well as teacher empowerment through collaborative teaming-promotes higher student outcomes. The researchers suggested additional studies about the level of principal participation in collaborative teams, teacher empowerment on intervention teams, and essential administrative supports for team efficacy and student outcome success.

People prefer habitual ways of doing things to new ways, and the deepest habits of people are embodied in the structure and culture of the organizations where they live out their lives. When change begins to touch on these deeply held habits and static assumptions, there is much more at stake than organizational effectiveness. Careers and feelings of personal worth are at stake, as are individuals' sense of social integration and belonging (Schlechty, 2001, p. 163). These insights are as true for schools as the expanding global society.

\section{Women in Leadership}

Of particular interest to this researcher is the role that women will play in the 
developing landscape of educational and instructional leadership. Although it is not the purpose of this study to investigate the presence, or lack of, women in educational leadership roles, it would be an omission of credible scholarship not to include this section, especially given that females have been involved in leadership activities throughout the history of the United States. Although not always called leadership, women's capacities to deal with difficult situations and to manage enterprises have earned them the reputation of being strong and resilient, capable of great initiative (Grogan, 2005).

Fennell (2005) conducted a two-year study of six women principals' lived experiences with leadership in schools. The purpose of the study was to juxtapose the daily routines and activities of the study participants to the theories of structuralist, constructivist, critical, and post-structuralist philosophies to gain an increased understanding of leadership in environments of change. The study participants were six of 12 principals from an urban school district in Northwestern Ontario, Canada. Since the study was focused on principals, participants with the widest and most varied experiences within the group of 12 were selected. Two were in the early part of their careers, two were mid-career, and two were nearing the end of their tenure.

The methodology used by Fennell (2005) was a review of related literature and a two-year phenomenological study of the principals conducted through six in-depth interviews 1-2 hours in length with each of the six participants at three-month intervals over a period of two school years, for a total of 36 interviews. Participants were encouraged to share critical incidents, in anecdotes, stories, or experiences about their leadership. Probing questions were used to encourage participants to discuss further illustrations that could lead to the saturation of the database for each area under discussion. The researcher read and checked interview transcripts for accuracy and as preparation for 
future interviews with each respondent. To provide triangulation for the qualitative research, field notes were also collected through observations of the principals in their respective schools. Following data collection, a more detailed analysis was used to isolate themes and concepts.

The lived experiences of the women principals centered on creating a positive, open, collaborative learning community inclusive of teachers, students, parents, and community members. The principals were good listeners, effective communicators, and superb negotiators. They emphasized valuing others as individuals and creating strong reciprocal relationships. The principals viewed power and knowledge as interdependent in developing a common language for individuals to use their expertise to share in the leadership and development of a learning community. They managed conflict by using principles of democracy, equity, and thoughtful reflection to deal with issues head on rather than viewing dissensions as differences in individual personalities (Fennell, 2005).

Fennell (2005) did not determine broad generalizations to larger populations; however, the examples and experiences described may provide a clearer understanding of the need to include philosophical perspectives in training programs for aspiring leaders. Implications for future research include examination of lived experiences of traditional and non-mainstreamed group members to encourage development of new models and metaphors for leadership. New paradigms for studying active leadership require such individualized research.

Fennell (2005) noted that women principals are "more attuned to teaching, curriculum and instruction, and children, perhaps because they spend more time as teachers and as mothers before they become administrators" (p. 488). From extensive literature, Fennell suggested that women leaders often focus more on relationships between 
individuals and communities and viewed power as a multi-dimensional and multidirectional process to empower others, rather than having power over them. Fennell concluded that for women's experiences and theories to become part of the main body of knowledge on leadership, they must be studied on their own terms rather than in relation to those based on experiences of predominantly white, male leaders.

Rosener (1990), with the help of McAllister and Stephens, conducted a study commissioned by the Leadership Foundation of the International Women's Forum, about women and men leaders, specifically the similarities and differences between male and female leaders. The focus was how women are developing their own rules of conduct regarding leadership styles by drawing on the skills and attitudes learned in their shared experiences. Women leaders are portraying attributes unique to their socialization as women and creating a different path to the top. The study participants were members of the International Women's Forum (IWF). Both men and women members were asked to participate; all were similar in age, occupation, and educational level. Club membership descriptions did not include gender-specific data. However, the IWF has some 37 forums in North America, Europe, Asia, Latin America, and the Middle East.

The methodology used was twofold: an eight-page questionnaire and interviews. Rosener (1990) conducted the interviews of some women IWF members. The selection process of who was interviewed was not provided. The respondents were asked questions about their leadership styles, organizations, work-family issues, and personal characteristics. The questionnaire response rate was $31 \%$. The findings from the questionnaires and interviews included: (a) Women earn the same amount of money as their male counterparts. The average yearly income for men was $\$ 136,510$; for women it was $\$ 140,573$; (b) The men's household income (their own and their spouse's) is much 
lower than that of the women-- $\$ 166,454$ versus $\$ 300,892$ (only $39 \%$ of the men have fulltime employed spouses, as opposed to $71 \%$ of the women); (c) Women are more likely than men to use transformational leadership--motivating others by transforming their selfinterest into the goals of the organization; (d) Women are much more likely than men to use power based on charisma, work record, and contacts (personal power) as opposed to power based on organizational position, title, and the ability to reward and punish (structural power); (e) Approximately $67 \%$ of the women respondents were married; (f) Both married men and married women experience moderate levels of conflict between work and family domains. When there were children at home, women experience only slightly higher levels of conflict than men, although they shoulder a much greater proportion of the childcare-- $61 \%$ of the care versus $25 \%$ for the men. One limitation to the study was that sample size information was not provided. Response rate percentage was given but not number of participants. Another limitation is that the respondents in this study were women executives and members of an organization of international reach and may not represent of the larger population of working women.

Jacobs and McClelland (1994) conducted a 12-year longitudinal study of men and women leadership motive patterns and managerial success in a large utility corporation. Using Thematic Apperception Test (TAT) data collected before entering management positions, sex-differences in motive patterns related to leadership were examined to determine whether the same motivational and personality characteristics that have, in the past, predicted managerial success in men also predict managerial success in women. The subjects of the study were 211 male and 180 female entry-level managers who participated in the Management Continuity Study (MCS), a long-term management assessment project initiated in a large utility company. Of the study sample, $68 \%$ were 
White, $24 \%$ were Black, $7 \%$ were Hispanic, and $1 \%$ was other (e.g., Asian, American Indian, etc.). Male participants ranged in age from 21 to 37 with a median of 26 , and females ranged from 21 to 43 with median 25. All participants had earned a Bachelor's degree.

The initial assessment in 1977 included a three-day assessment; the TAT was administered along with self-report instruments, behavioral simulations, and an interview (Jacobs \& McClelland, 1994). Stories written by the participants were coded for the following motives: n-Achievement, n-Power, n-Affiliation, and AI-Activity Inhibition. Motive scores were corrected for protocol length using the regression equation. The residual motive scores were saved and transformed to T-scores $(M=50, S D=10)$. Participants were categorized as having Leadership Motive Patterns if: (a) their T-score of n-Power was greater than or equal to 45, (b) their T-score of n-Power was greater than or equal to their T score for n-Affiliation, or (c) their Activity Inhibition score was equal to or above their median for the sample. Follow-ups occurred every two years through 1986. During 1990, subjects again were located and contacted. Of the 391 participants, 126 males and 103 females were still actively employed with the utility company (Stayers). Of the participants who left the company (Leavers), current job status was available on 31 males and 25 females. In 1990, participants completed a questionnaire about their current job status. Sixty-two percent of the MCS sample was actively employed with the utility company (Stayers), with 1 participant deceased. Of the 147 subjects who left the utility company's employment (Leavers), information was not available for $37 \%(n=54)$; however, of the remaining 93 Leavers information was available for $72 \%(n=67)$. Jacob and McClelland (1994) conducted chi-square analyses to compare the Stayers $(n=231)$ and Leavers $(n=67)$ samples on demographics, attained management 
levels, and motive scores. Findings indicated no significant difference by sex or age, meaning that the achievement motivation, which was found in earlier studies to predict management potential for males, also predicted middle management potential for females. The study confirmed that women who are successful in management are as career motivated and interested in status attainment as men.

Owen (1986) conducted a study on the emergence of female leaders to determine if such women in heterogeneous groups utilize a prescribed process. Owen used three leaderless mixed-sex task groups in an upper-division communication course in a private university in the northwestern United States to guide the study: "What are the rhetorical themes used by females who emerge as leaders of small task groups?" Twenty-one junior and senior non-communication majors comprised the three study groups formed during the first week of the semester class. Students were asked to form groups of their choosing with only one criterion: an almost equal number of females and males in each group. Seating proximity guided most group formations with some students selecting groups because of previous acquaintances. The 12-week assignment entailed the completion of a groupselected task of solving or improving an aspect of campus life, and a presentation of the results to the class at the end of the semester. Group 1, composed of three females and five males, chose to design and present a campus-wide career orientation fair. Group 2, two females and five males, conducted a school alcohol use survey. Group 3, two females and four males, investigated potential improvements of the student union building.

Owen (1986) employed non-participant observation of group meetings, face-to-face and telephone conversations with group members, on-going group written logs, and analysis papers for data collection. Each member kept a $\log$ in which he or she reported the group's progress, physical setting, group roles, interactions patterns, leadership 
development, and feelings about the group. At the end of the semester each group member also wrote an 8- to 10-page paper as a cumulative analysis of the group experience. The 544 pages of written logs and analysis papers were the primary data of the study.

Owen (1986) clustered the texts by groups and interpreted the data with an emphasis on the common and discrepant views of leadership with specific focus on the interpretive themes of conversation and perceptions of group activity through the eyes of the eventual leaders of the groups. The three females who emerged as group leaders held in common two rhetorical themes: (a) the rhetoric of the "hard worker" and leading by default, and (b) the rhetoric of "not leading" (Owen, p. 483).

Tentative interpretations by Owen (1986) suggested the emergent female leaders maintained a subtle, yet hard-working ethic, that is, they simply outworked the other group members by accepting more responsibilities. The emergent leaders strengthened their leadership roles by consciously striving to be the "organizers" and "coordinators" rather than accepting the stigma of "leader," thus inviting competition for the role (p. 484).

Storey and Zellinsky (1993) conducted a qualitative study on the perspectives of the first few years of school leadership for women. The study was the preliminary phase of an initiative supported by the Ministry of Education's Gender Equity Staff Development Fund to prepare professional development activities to meet the needs of women aspiring or currently in educational administration positions. The sample selected for the study consisted of 14 women and 9 men: one director of instruction, one high schoolprincipal, three elementary principals, one high school vice-principal, and 16 elementary viceprincipals. Almost half had master's degrees in administration, curriculum and instruction, or supervision. The combined teaching and administrative experience ranged from 11 to 25 years with a mean of 17 years. 
Interviews were conducted with each participant. The interviews were audio-taped and transcribed. Each interview began with reflective questions regarding current thoughts about the decision to be an administrator followed by focused questions on events and circumstances that influenced the participant's decision to become an administrator. The interviews moved to questions about challenges, obstacles, and barriers encountered, if any, then to an examination of common experiences to discussion of mentors and networks. Storey and Zellinsky (1993) indicated that most of the participants (17 out of 23) enjoyed their positions very much. On a five-point scale, eight of the men and nine of the women rated their decision a score of five. Variation existed between men and women as to the circumstances leading them to seek an administrative position. For men, existing norms encouraged a career in school leadership while several women acknowledged being influenced by their principal, teaching colleagues, or spouses. Most respondents did not mention overwhelming obstacles or challenges although some candidates expressed concern about the potential impact on their families. The women gave specific indications, generally in humor, that in some situations, barriers exist. However, most felt little outright discrimination because they were female. The study participants all described support networks intentionally developed and maintained. Most subjects networked with male and female peers although some women connected more regularly with other women. Storey and Zellinsky (1993) proposed a professional development model for aspirant leaders. The two most important factors for the beginning administrator were mentoring and networks. The model also suggested four stages in the move toward leadership: early consideration, application and acceptance, approach, and commencement.

Dorn, O'Rourke, and Papa-Lewis (1989) conducted a qualitative study of nine female administrators' stories of their worlds, qualities of leadership they felt important, 
and their perspective of differences in male and female leadership styles. The outcome of their research was "a view of the world from a female's perspective" (p. 24). Methodology used was an interview protocol designed specifically for this study. Ten open-ended questions were developed to gather information about thoughts, feelings, and perceptions as related to: (a) "requisite qualities of effective female educational leaders; (b) difficulties experienced; (c) differences in ways men and women work; and, (d) significant life experiences" (p. 3). Nine female administrators from San Joaquin Valley, CA, were interviewed during the Spring of 1989--2 district superintendents, 2 special education principals, 1 high school vice principal, 1 curriculum coordinator, and 3 elementary principals. Each interview lasted one to two hours, with five of the nine participants agreeing to be audio-taped.

Data analysis by the researchers was both qualitative and descriptive. Data consisted of information from the interviews and the answers given for the 10 interview questions which were sorted into categories. Dorn et al. (1989) discovered two themes: leadership qualities and perceived differences between the way men and women lead. The nine female administrators consistently thought the following qualities were most important to them as leaders: integrity, positive thinking, patience, hard work, enthusiasm, listening, and organization. Other qualities viewed as important were caring, nurturing, inclusiveness, intuition, and openness. In extant literature, these qualities are identified as feminine. The nine female interviewees in this study described the differences in the ways men and women work as "women have to be more skillful, while men rely on image, power brokerage, and associations; men are more task-oriented, women are more idealistic; men are detail-oriented; women are more nurturing and caring; men are authoritarian and women are participatory" (p. 26). Implications for further study are life 
experiences of women in school administration and administrator training programs.

This section reviewed leadership as an examination of what leadership means followed by how the concept of leadership is changing as the tenets within our society are changing. Leadership is no longer one person dictating the agenda of the culture; the more successful leaders encapsulate a collaborative team concept that allows empowerment of the stakeholders. The section concluded with leadership styles and experiences of women. The next section considers educational leadership as it continues to evolve along with dynamics of global perspectives.

\section{Educational Leadership}

Before the educational reforms initiated in the $1980 \mathrm{~s}$, portraits of educational leadership were heavily classroom focused; they featured practices intended to influence classroom curriculum and instruction directly (Beck \& Murphy, 1993). Such a classroom focus was a reasonable response to both the educational reform agenda of the time (the effective schools movement) and the improbability of prevailing school administrator practices to push the agenda forward (Leithwood, 1994). However, the 1980s brought a new surge of interest. During this period, the number of empirical investigations into educational leadership effectiveness increased significantly. Research reviews of the time encouraged a focus on the effects of principal leadership (Hallinger \& Heck, 1998) inclusive of the direct and indirect district level posture shaping principalship practices. The following review of educational leadership reiterates the building level administrator, the principal, as bearing the responsibility of instructional leadership focused on student success but with influential direction from educational leadership at the district level.

Myers and Murphy (1987) conducted an empirical study that explored the influence of supervision as an administrative control mechanism used by district level administrators 
to direct the work of high school principals, examining the nature of control in high schools. Most of the data regarding the supervision of principals had been gathered in elementary schools. Due to the differences between elementary and high schools in organizational variables such as size, student characteristics, faculty characteristics, curricular complexity, departmental structure, school goals, and parental relationships, more research directly on the supervision of high school principals was needed. The specific focus of this study was on the supervisory control function. Two questions guided the study: (a) what patterns of supervision are used by district offices in working with high school principals? and (b) what do these patterns suggest about the nature of control used to shape the work of high school principals? Peterson (as cited by Myers \& Murphy) developed the theoretical framework that informed the study. The Administrative Control Model that guided the work was adapted from Murphy, Hallinger, and Peterson (1986) and Murphy (1987) and illustrated how the district office personnel constrain the work of principals through a series of control mechanisms. The purposes of this study were to add to the empirical research literature about the supervision of school managers and to focus directly on the supervision of principals at the high school level.

The sample was selected from suburban K-12 units and high school districts (grades 9-12) in northeastern Illinois and stratified by district size (number of schools in the district). The analysis of the schools' socioeconomic status data reflected a relatively homogenous population and therefore nullified the use of socioeconomic status as a variable. The suburban communities represented were upper middle class (Murphy et al., 1986). The participants were 12 white male principals ranging in age from 40 to 60 years, with a mean age of 51 . Nine of the 12 principals had earned doctorate degrees, one was working on a dissertation, and two of them had master's degrees with postgraduate credit. 
All principals had teaching experience that varied from 4 to 18 years.

Procedures used in the study examined the perceptions of high school principals of how suburban superintendents used supervision to shape the principal's work in the administrative aspect of the principalship. Two factors, socioeconomic status and district size, were examined along with balance of control and autonomy provided to principals by superintendents. The research design used two instruments developed by Peterson (as cited in Murphy et al., 1986)--a survey questionnaire and an interview protocol--which were originally designed for elementary school principals but adapted by minor changes for use in high school districts. The original survey and the interview questions were validated through a process in which an extensive interview protocol was written for and administered to a group of superintendents, transcribed and analyzed, then compared with a principal's interview and evaluated for validity and reliability. A field-test of the Peterson survey and interview protocol was conducted on two high school principals as a check for appropriateness of usage for high school principals. The researchers found that Peterson's survey could be adapted, with minor changes, for use in high school districts.

The 35-question interview protocol consisted primarily of open-ended questions and required one and one-half to two hours to administer. Each interview was audio-taped and transcribed. The survey questionnaire was designed to collect demographic data and was explained to the respondents at the interview session. Participants returned the surveys on a later day. The data were analyzed using guidelines recommended by Goetz and LeCompte and procedures developed by Miles and Huberman (both cited in Myles \& Murphy, 1984). The questions from the survey instrument and the open-ended questionnaire that focused directly on the supervision control mechanism were used to develop a coding matrix. The matrix contained columns with information on each school, 
such as identifying information for the interviewee, district number and type of school district, size of district, socioeconomic status, enrollment, and test scores. The districts were grouped by size (number of schools in the district: (a) large, four or more schools, (b) medium, two or three schools, and (c) small, one school). Each principal's answer to each question was analyzed individually for that specific question and then analyzed for each size category--large (four or more schools), medium (two or three schools), or small (one school) school district--and for each district type. Counting and clustering were used as part of the theme analysis.

The control mechanisms used in the study were supervision, defined as a visit from the central office personnel to a principal's school; size and type of district; proximity; sources of information in the supervision process; and importance of supervision and evaluation. The findings indicated that supervision, the most prevalent control mechanism, was used in all schools to varying degrees. The frequency of central office personnel visits fluctuated between almost every day, in the case where the central office personnel and the principal shared the same facility, to twice per year. District size did not influence the frequency of visit frequency. Schools of both large and small districts received roughly the same number of visits. Ninety-two percent received a medium or high numbers of visits. Most principals perceived visits by the central office staff as non-threatening; however, when they did visit they collected information about all aspects of schooling. Usually, principals perceived supervision as light, and felt that schools and principals were afforded considerable autonomy.

Murphy and Hallinger (1986) conducted a study that examined how district level administrators in effective school districts exercise instructional leadership. Concepts from school effectiveness studies and from organizational literature on coordination and control 
were employed for understanding how district personnel organize and manage instruction and curriculum in these effective districts. Specific instructional management practices are examined within a framework of six major functions: (a) setting goals and establishing expectations and standards; (b) selecting staff; (c) supervising and evaluating staff; (d) establishing an instructional and curricular focus; (e) ensuring consistency in technical core operations; and (f) monitoring curriculum and instruction. The purpose of the study was to describe the educational leadership patterns employed by the sample population of district personnel and the strength of coupling (establishing clear goals with inspection of processes and outcomes, i.e., accountability) in effective organizations.

The study by Murphy and Hallinger (1986) utilized qualitative data analysis collected from the 12 educational leaders in school districts in California identified as instructionally effective school districts (IESD). The designation of IESD was determined by the consistent performance of exceeding the expected range of student achievement, based on student socioeconomic demographics, in reading, mathematics, and language arts over the three-year period of 1980-83. Random sampling was not used since the sample size would be too small to make statistical tests aimed at generalizing results insignificant. Methodology included the development of a scheduled standardized interview instrument to assess the educational and instructional leadership roles of district level leaders. Questions were primarily open-ended and organized under nine control functions: (a) selection; (b) socialization; (c) supervision; (d) evaluation; (e) rewards/sanctions; (f) goals; (g) resource allocation; (h) behavior control; and (i) technological specifications. Interviews were conducted with the leaders of each district in their offices during July 1984. The audio-taped interviews lasted an average of 2.5 hours with the researchers spending an equal amount of time after each interview reviewing and clarifying notes. The 
districts were asked to provide the following data: (a) district goal statements; (b) principal evaluation forms and sample evaluations; (c) district newsletters; and (d) district organizational charts. Data were assimilated into a descriptive matrix and then analyzed: (a) individually across control functions to determine themes, factors, and characteristics of instructional leadership; (b) as a group to determine if themes were evident across the sample; and (c) vertically for subsuming conceptual and theoretical references. Document analysis validated self-reporting.

Murphy and Hallinger (1986) indicated that the district leadership was actively involved in managing and directing technical core activities within the districts using direct and indirect leadership tools for controlling the development of goals at both the district and school levels. District leadership also was influential in establishing procedures for the selection of staff and took personal responsibility for the supervision and evaluation of principals. In addition, the district leaders appeared to use tight coupling (accountability) in curriculum and instruction. Limitations of the study included the definition of effectiveness solely focused on students' test scores and the implicit acceptance of the assumption that leadership is a cause of district effectiveness.

Hallinger and Murphy (1995) conducted a study in which they examined the behavior of a group of 10 school principals in a working class district in a suburb of California. The superintendent of this district had been attempting to promote instructional leadership through a variety of district level policies and practices. Over a four-year period, the superintendent drew upon research to design and implement a plan to promote instructional leadership among his principals. The purpose of the investigation was twofold: (a) to describe what principals in the district were doing with respect to managing curriculum and instruction, and (b) to begin to examine the impact of the superintendent's 
policies for promoting instructional leadership. A questionnaire instrument, the Instructional Management Rating Scales by Hallinger (cited in Hallinger \& Murphy) was used to collect the data from the teacher population $(N=104)$ with the goal of determining teachers' perceptions of principals' instructional leadership. The questionnaire was divided into three main categories: (a) defines the mission; (b) manages the instructional program; and (c) promotes school learning climate. The questionnaire also contained eleven subscales and seventy-one items. A single response, five-point Likert scale, ranging from (1) "almost never" to (5) "almost always" was used. The adequacy of the instrument satisfied rigorous reliability and validity standards as the following five criteria were applied: (a) content validity; (b) Cronbach's alpha; (c) analysis of variance; (d) construct validity; and (e) construct validity.

Calculated mean scores and standard deviations for each of the instructional subscales showed the group of principals with a high level of instructional leadership behavior and activity. The principals were strong in performance on the two subscales with the dimension "defines the school's mission" as well as the dimension "promoting a positive school learning climate." The highest rating for the principal groups was "managing the instructional program" (Hallinger \& Murphy, 1995). The high level of instructional leadership behavior performed by the district's principals came as somewhat of a surprise to the researchers in light of observational literature. They offered at least three possible explanations for this discrepancy: (a) structured observational studies underestimate the amount of instructional leadership behavior performed by principals; (b) the instrument used in the study may have overestimated the instructional leadership behavior performed by principals; or (c) factors unique to this school district. The researchers credited the high level of instructional leadership behavior of the principals to 
district superintendent's policies and suggested the next step of future research on principals must be to examine the nature of the superintendent-principal relationship so that more useful guidance be given to that aspect of their jobs.

Murphy (1994) conducted an exploratory study of the changing role of the superintendency considering the major efforts to reform education throughout the nation since the mid-1980s. The purpose of the research was to sharpen the boundaries and landmarks of the effects of restructuring invoked on the role of central office administrators, specifically the superintendent. Four possible scenarios under transformational reform efforts are analyzed: (a) almost no change in the role; (b) the demise of the position; (c) the role functionally redefined; and (d) the position will undergo a metamorphosis rather than a restructuring. The task of choosing a target population focused on districts involved in serious transformational reform efforts--those involving significant shifts in the organization, governance, and management of schools; the work of educational stakeholders; and the learning and teaching process. Given the slowness of the restructuring movement to affect central office operations in the United States, the researcher chose a state in which a radical overhaul of schooling was in progress. Because the Kentucky Education Reform Act of 1990 (KERA) represented "the most comprehensive education reform legislation in the nation" (David, as cited by Murphy, 1994a), the target population became the 176 school district superintendents in the Commonwealth of Kentucky. Of the target group, 78 responses were received; seventyfour responses were usable.

For the study, Murphy (1994) developed a questionnaire instrument consisting of eleven open-ended questions addressing five topics: (a) the major purposes of restructuring in Kentucky; (b) the effects of restructuring; (c) the ways in which restructuring was 
shaping the superintendent's role; (d) changes in district office operations and roles because of restructuring; and, (e) "the good, the bad, and the bothersome" (Murphy, p. 350) about change efforts across the state. The data from the questionnaire protocols were analyzed to discern the superintendents' perceptions of changes taking place in district offices and in the role of the superintendency. Methodology used to analyze data (phrases, sentences, and paragraphs) provided coded responses that were deductively and inductively similar to interview transcripts. Groups of codes were utilized as: (a) eleven codes consistent with the research questions; (b) an attachment of chunks of material to relevant sections throughout the responses; (c) an aid in classifying the relationships of the superintendents to teachers, principals, and district personnel; (d) an understanding of a superintendent's responsibilities; and, (e) categorical development of themes. Constant comparative methodology led to seven taxonomical categories subsumed by three larger domains of developing community, coaching from the sidelines, and struggling to meet the KERA mandates.

Although KERA of 1990 decentralized control over education to local communities, the data collected from the 74 Kentucky superintendents provided almost no support for the view that the superintendency would remain unchanged or be eliminated. New roles are emerging and are being played out with varying degrees of reluctance and willingness. Limitations to the study included: (a) data were self-reported; (b) no efforts were made to interview non-respondents; and (c) there were no formal member checking procedures to secure feedback from informants. Murphy (1994) suggested that future research may build on this current work by expanding the array of stakeholder groups and forms of data collection.

Educational reform analysts in the United States over the last couple of decades 
typically generalize the transformations of the educational arena between a top-down set of initiatives to an assortment of measures designed to empower people (Murphy, 1994). Common elements include efforts to decentralize decision-making; empower parents, students, and teachers; devise new forms of organization, governance, and management of schools; and develop new roles and responsibilities for all members of the school community (Elmore, 1989; Murphy, 1991).

Instructional Leadership

As the research base related to principal effectiveness continued to highlight the significance of principal leadership as it relates to student achievement, reform initiatives of the early 1990s often emphasized the principal's responsibility for instructional leadership as opposed to the district superintendent. The re-emergence of the principal as the school's instructional leader is directly attributable to the effective school's research (Hallinger \& Murphy, 1986). Several researchers--Edmonds, Rutter, Brookover, Lezotte (as cited by Hallinger \& Murphy) all found that instructionally effective schools are characterized by strong instructional leadership typically present within the school building. Although research from related areas (e.g., management, change theory, implementation) as well as conventional wisdom emphasized the central role played by principals, it was not until the effective schools findings were disseminated that policy makers, district administrators, and leadership trainers began to focus on the principal's role in coordinating, developing, and controlling instruction. The convergence of research findings related to the leadership role of the principal has proven so powerful that school district practices for principals are now becoming shaped by the expectation that principals will act as instructional leaders for their schools (Hallinger \& Murphy). This view also turned into an important piece of the accountability model as principals bore more of the 
responsibility for school success.

Heck, Larson, and Marcoulides (1990) conducted an exploratory study to examine the means by which instructional leader behavior at the school level may affect school procedures and student academic outcomes. The study proposed that the manner in which the principal governs the school's political environments will directly affect school climate and organizational integrity, including instruction, which will impact student achievement. The sample for the study included all public elementary and high schools in the state of California that had either scored above or below their "comparison band" (p. 102) on the California Assessment Program (CAP) at the third- and sixth-grade levels or twelfth grade in reading and math for three consecutive years. The CAP is a basic skills test requirement for all students by the California State Department of Education. The "comparison bands" were determined by multiple regression statistical procedures to standardize differences in socioeconomic status and language background factors present in the student population. Criteria set by the researchers for participant participation were that the current principals of the schools had to be present at the school for at least three years, the principal and at least four teachers at the school had to return the survey instruments, and the schools had to have a three-year performance above or below their comparison bands. The final sample included teachers $(n=168)$ and principals $(n=30)$ in 30 schools.

Data were collected by Larsen's Instructional Activity Questionnaire that consisted of variables measuring how often 34 principal specific instructional leadership behaviors occurred (as cited by Heck et al., 1990). Nationally known educational experts analyzed the list of behaviors to determine the content validity of the instrument. A field test to a chosen group of principals and teachers was also conducted. Although the data collection included Larsen's 34 instructional leadership behaviors for principals, the researchers used 
22 of the 34 behaviors that they believed to be most strongly identified with instructional leadership. All subscales had internal consistency coefficients from .7 to .9 . The questionnaire was a five-point Likert-type scale with responses ranging from "never" to "always." The questionnaires were mailed to principals of 118 schools with instructions for the principals to give the questionnaires to a random sampling of six teachers in the school. The resulting sample was 168 teachers and 30 principals.

Data analysis consisted of means, standard deviations, and significance levels of $t$ tests, inclusive of individual and school level analyses. Findings included: (a) the principal's role in establishing school climate and instructional organization strongly predicts school achievement outcomes; (b) in higher achieving schools, principals involved teachers in instructional decisions to a greater extent than in low achieving schools; (c) principal leadership regarding school climate and instructional organization are equally important on student achievement; (d) the extent to which principals protected the staff from outside pressures, such as community and central office, might be an important principal governance issue; and (e) the principal's ability to manage the school's organization regarding instruction directly and strongly affects school climate (Heck et al., 1990). Due to the nature of this study, the research "provides support for the theory that principal instructional leadership is directly related to the school's performance at a higher or lower academic level and must now be considered as one 'school effects' variable that directly influences student achievement" (p. 121). An implication of the study is the importance of principals spending time in classrooms directly supervising the work of teachers. A limitation of the study is that it is an outlier study rather than a random sample and the middle range of schools was not a part of the study. Thus, a need for additional research would be to duplicate the study for the entire range of schools. 
The Accountability and Standards Movements

Two questions that need to be considered for any accountability system (Linn, 2003, p. 3) are: (a) What counts? and (b) Who is held accountable? Accountability systems focus their attention on student performance in addition to holding schools accountable either for students achieving at a certain performance level or for increasing performance from one academic cycle to the next (Fuhrman, 2004). Tests scores and other measures are collected to determine if their goals have been achieved specific to minimum standards. These systems also include consequences that range from rewarding high achievement to mandating intervention in low-performing schools.

Accountability systems are designed to assess the effects of education on students. They are presumed to measure, when designed appropriately, what students learn and the skills they develop through schooling. These accountability premises rely on five basic assumptions: (a) performance, measured as academic achievement, is the most important goal of schooling; consequently accountability systems must focus on outcomes; (b) the instruments designed by the systems can appropriately measure performance with accuracy and reliability; (c) the consequences are powerful enough to motivate both students and school staff; (d) because of this motivation, instruction will be more effective and performance will improve; and (e) unexpected and undesired consequences are minimal or pose no real threat to the systems (Fuhrman, 2004, p. 8).

Chance and Anderson (2003) conducted an interpretative study of high school principals' and high school science teachers' perceptions of the impact that new science standards enacted by the Nevada Education Reform Act have had on six areas: (a) instructions, (b) curriculum, (c) assessment, (d) accountability, (d) professional development, and (e) supervision. The researchers stated their main focus was on the 
instructional leadership activities of principals and how they were implementing the new Nevada state standards. Questionnaires were distributed to science teachers $(N=425)$ and principals $(N=130)$ in all Nevada public high schools $(N=65)$ in rural, suburban, and urban areas of the state. Forty-three high schools (66\%), 196 of the science teachers $(46 \%)$, and 56 administrators $(43 \%)$ responded.

Data were collected through questionnaires and semi-structured telephone interviews (Chance \& Anderson, 2003). The Nevada Science Standards Questionnaire was developed based upon common, emerging themes from research on instructional leadership and geared toward the goals of curriculum standards as formulated by the Nevada Council to Establish Academic Standards for Public Schools. One form of the questionnaire was developed for administrators and another for teachers. The questionnaire consisted of seven demographic questions and 49 questions related to how the science standards had been implemented and what impact the standards had on classroom practices. Interviews were conducted with randomly selected principals and teachers to triangulate data from the questionnaires and to provide detailed descriptions of their perceptions. Data from the questionnaires were analyzed using descriptive analysis statistics and $t$ tests for determining significance of the means. Interview data were analyzed through domain analysis.

Chance and Anderson (2003) found that administrators and science teachers' perceptions of the impact of Nevada science standards differed significantly in all six areas (instruction, curriculum, assessment, accountability, professional development, and supervision) related to instructional leadership. Questionnaire data were used to report specific findings. Additional findings included: (a) in instruction, only $46 \%$ of science teachers' perceived standards had a positive impact whereas $77 \%$ of administrators 
perceived a positive impact; (b) in curriculum, $49 \%$ of science teachers perceived that Nevada science standards matched their goals for what they want and expect from science curriculum, whereas $78 \%$ of administrators responded favorably; (c) in assessment, $83 \%$ of administrators thought the standards helped improve academic achievement of students while only $42 \%$ of the teachers did; (d) in accountability, $17 \%$ of science teachers and $27 \%$ of administrators agreed or somewhat agreed that the standards assisted administrators in holding parents accountable for student learning; (e) in professional development, $43 \%$ of science teachers responded favorably while $86 \%$ of administrators responded favorably concerning the impact of standards in promoting group development among science teachers; and (f) in supervision, less than $37 \%$ of teachers responded favorably that science standards assisted classroom supervision while more than $60 \%$ of administrators responded favorably.

Chance and Anderson (2003) concluded that: (a) administrators and science teachers seem to have a different frame of reference regarding the impact of science standards in Nevada high schools; (b) principals viewed standards as having a greater impact on curriculum and instructional practices than did teachers; (c) principals perceived their behaviors to be more supportive of teacher collaboration and more encouraging of dialogue than did teachers; and (d) what principals saw as leading, coaching, encouraging, and supporting was not viewed that way by teachers. The researchers suggested principals should reflect carefully upon their own leadership behaviors to have a positive impact on instructional improvement and curriculum reform. A limitation of the study is that the researchers did not inquire why the teachers were so much more negative.

In 2002, Ladd and Walsh conducted an evaluation analysis of the value-added approach to measuring school success and its implementation as a tool for increasing 
student achievement. Attempting to hold schools accountable, some states calculate and publicize value-added measures of school effectiveness in documents such as school report cards. Additionally, states such as South Carolina, Kentucky, and North Carolina offer financial rewards for personnel in schools that are performing well and apply sanctions to schools that are performing below standard requirements. However, some of these measures fail to account for measurement error, creating negatively biased data toward schools serving a large concentration of disadvantaged students. Consequently, high quality teachers and administrators may try to avoid schools serving low socioeconomic status (SES) students, favoring schools serving high SES students. The purpose of the analysis was twofold: "(a) to contrast such measures as those employed by South and North Carolina to indicate school effectiveness and relegate incentives to value-added measures that would more accurately measure a school's efficiency; and, (b) to evaluate such measures on their own terms, that is, as measures of school-specific gains in student performance" (p. 2).

The data set used by Ladd and Walsh (2002) to demonstrate considerations statistically in measuring value added was a 3-year set of more than 37,000 students' test scores in North Carolina. The test scores were for fifth grade students in reading and in math matched to their third and fourth grade scores. Birthday, gender, and school matched students' scores. This process was imperfect in that for all students who transferred from one school to another during the 3-year period; their data were lost for purposes of the analysis. Also, in the matching process, any students coming to a school from out of state anytime during the school year would be excluded from the measure of a school's effectiveness. Test data were matched and calculated for one randomly chosen sub-sample of 763 elementary schools and the entire sample of 997 schools. There were no substantial 
differences between the two samples. A limitation of this selection process is that more mobile (typically lower SES) students are systemically excluded from the database.

North Carolina and South Carolina measure school effectiveness as the "median of the differences between actual test scores and predicted test scores (South Carolina) or as the mean of the residual differences between actual and predicted changes in test scores" (North Carolina) (Ladd \& Walsh, 2002, p. 8). Neither state included school specific indicator variables in their model. School specific indicator variables would correct for such phenomena as higher-ability students clustering in schools deemed more effective and lower-ability students clustering in schools with lower intercepts in the fixed effects model. In this model, parameters for test scores are estimated by regression analysis based on all students for whom both current and prior-year test data are available.

The researchers suggested three plausible explanations: (a) schools with higherability students may in fact be more effective than schools serving low-performing students due to availability of "more resources, they may generate greater positive peer effects, and they may attract higher quality teachers who can use their seniority to move to schools where the students are more motivated and easier to teach" (Ladd \& Walsh, 2002, p. 5); (b) the equations used to calculate school effectiveness are statistically mis-specified; or (c) the equations statistically could be subject to measurement error.

To determine if specification error caused negative bias of schools serving higher and lower SES students, the researchers compared rankings of schools using mean residuals of student test scores versus fixed effects with school-specific intercepts. Ladd and Walsh (2002) concluded the residuals approach ranked schools' average student ability too low or too high relative to their true ranking. The researchers then attempted to eliminate measurement error by using the method of instrumental variables. This procedure 
correlated the third grade scores to fourth grade scores, thereby, yielding consistent parameter estimates. They then used a Wald test to check for the presence of measurement error. The test clearly indicated that measurement error is a serious problem. For example, the chi-squared statistic for math using the South Carolina model is 2508 , which far exceeds the critical value of 12.59 .

With this study, the researchers hoped to emphasize the importance for states or school districts to supplement any test-based accountability systems with other policies designed explicitly to improve the outcomes of students in schools with large percentages of low-performing students. "Otherwise, test-based accountability systems could lead to a significant widening of the achievement gap between low-performing and high-performing students" (Ladd \& Walsh, 2002, p.16).

In Washington State after the first elements of the new state educational system were in place, Lake, Hill, O'Toole, and Celio (1999) conducted a survey and analysis of two statewide samples of schools. The state's Commission on Student Learning identified student learning standards and, based on these standards, designed tests to determine whether individual students, schools, school districts, and the state as a whole were meeting the standards. The state also committed to a set of actions to help struggling schools, eliminate regulations that reduce school effectiveness, and help teachers do their jobs better. The purpose of the study was to compare the two samples of schools and determine why one sample of schools met or exceeded the standards while the other sample did not meet state standards.

The methodology employed by Lake et al. (1999) was a survey of two statewide samples of schools whose students took the same grade level test for two consecutive years--both took the tests in 1997 and 1998. The first sample was 30 schools whose scores 
improved significantly; the second sample of schools improved only slightly or not at all. Both school groups were similar in demographics and in the same parts of the state. Principal interviews of 35 of the 40 schools were conducted on the phone with the interviews lasting an average of 30 minutes. Written materials about each school's improvement strategy were also collected.

Lake et al. (1999) found that the schools making significant gains in test scores took a pro-active approach toward improvement. Principals and teachers assessed strengths and weaknesses, set a few priorities, focused on improving instruction, and took initiatives to find the help the school needed to achieve its goals. The principals and teachers in these schools re-allocated funds, rearranged teacher work assignments and instructional schedules, and made sure all staff members coordinated their classroom work. Further, the improving schools continually and straightforwardly assessed their own progress.

Lake et al. (1999) identified certain attitudes and practices common in improving schools: eagerness to improve, acceptance of responsibility, willingness to take initiative, determination to unify the efforts of teachers, and openness to new roles for parents (p. 16). An implication of the study is for this kind of research to continue in subsequent years. Additionally, in education reform, no single formula is best for every school and student.

\section{Leadership Standards}

In the current era of accountability, considerable attention has been devoted to finding ways to improve the quality of leadership in our schools and school systems. To date, a good deal of energy has been focused on restructuring the training programs that prepare educators for formal leadership positions generally via a state licensure system (Murphy, Yff, \& Shipman, 2000, p. 17). Educational leaders need a clear understanding of what is expected and what is valued along with the personal and organizational resources 
to pursue valued goals. In response to the need, in 1996, a consortium of state and professional associations, the Interstate School Leaders Licensure Consortium (ISLLC), published a comprehensive set of Standards for School Leaders.

Seven principles helped guide the work of the consortium:

1. Standards should reflect the centrality of student learning.

2. Standards should acknowledge the changing role of the school leader.

3. Standards should recognize the collaborative nature of school leadership.

4. Standards should be high, upgrading the quality of the profession.

5. Standards should inform performance-based systems of assessment and evaluation for school leaders.

6. Standards should be integrated and coherent.

7. Standards should be predicated on the concepts of access, opportunity, and empowerment for all members of the school community. (Murphy et al., 2000, p. 17)

The ISSLC document contained six standards, each with specific indicators identifying the goals in three distinct areas of leadership: knowledge, dispositions, and performance. The six ISLLC Standards focus on the essential aspects of leadership-defined in relation to student achievement. The ISLLC Standards are listed in Appendix B.

According to a study conducted by Murphy et al. (2000), the ISLLC is widely accepted across the nation. Some critics argue however that ISLLC lacks enough specificity and operational guidance to help school leaders use them for action. Others claim the standards are not anchored in a professional knowledge base or rigorous research (English, 2000; Hale \& Moorman, 2003).

The Waters and Kingston (2005) research study examined the findings from the Mid-continent Research for Education and Learning (McREL) comparative analysis of the 
Balanced Leadership Framework (Waters, Marzano, \& McNulty, 2003) and the Interstate School Leaders Licensure Consortium (ISLLC). Strides made in the educational leadership research on what is essential for school leaders to know to improve student achievement rendered concern about the current and future states of leadership, in particular the principalship. The purpose of the study was to provide insights about what future standards or principals should encompass based on the most current research on school-level leadership. Research concerns were: (a) Who will lead schools of the future? (b) How can we retain current school leaders? (c) How can we attract new leaders? (d) How and by whom should school leaders be prepared? (e) What should they know and be able to do? Waters and Kingston (2005) noted that the methodology used by ISSLC and the national Policy Board of Education to identify leadership responsibilities, functions, and practices for school leaders involved an international effort. These efforts produced important leadership responsibilities; however, attempts to formalize a manageable scope of principal responsibilities rendered a wide range of responsibilities. In 1996, the ISSLC and Policy Board published the six Standards for School Leaders with 184 indicators. Methodologies employed by McREL to identify leadership responsibilities, functions, and practices for school leaders were meta- and factor analyses. The McREL comparative analysis used the six key findings of the McREL Balanced Leadership Framework to compare the 184 ISLLC indicators to the 66 leadership practices associated with the 21 leadership responsibilities identified in the meta-analysis.

The findings in the Waters and Kingston (2005) report described insights gleaned from the McREL analysis. Although elements of many of McREL's leadership practices were found in the ISLLC indicators, there were very few cases of a one-to-one correspondence of a leadership practice and an indicator. The factor analysis identified the 
McREL factors as first- and second-order that may aid in the prioritization of essential and important leadership responsibilities associated leading change and shared leadership with student achievement. A limitation of the study was the evidence of non-explicit associations between the practices and indicators that were beyond the scope of the analysis.

The Commonwealth of Kentucky in response to ISLLC and related research adopted standards for administrators by statute in what is commonly called the Effective Instructional Leadership Act (EILA)--Section 156.101 of the KRS 158.6455 passed by the General Assembly in 1998 (Ecton cite).

The Kentucky Education Reform Act

The Commonwealth of Kentucky enacted the Kentucky Education Reform Act (KERA) in 1990. KERA was passed by state legislators as the result of a lawsuit filed by 66 Kentucky school districts. These districts challenged the state's funding formula deemed as relying too heavily on local resources, resulting in inequitable educational opportunities for the state's school children. Because of the lawsuit, the Kentucky Supreme Court ruled the state's educational system unconstitutional. The passage of KERA, with the aim to provide a means to improve education, was heralded as the most comprehensive education package ever passed by a state legislative body (Steffy, 1993). The basis of the reform was to offer high quality public education for all children and to prepare students for success after graduation. To perpetuate the goals of increased student achievement, Kentucky Department of Education (KDE) through KERA, established accountability guidelines for schools (Steffy), designed in ways consistent with current theory, research, and experience (Fuhrman, 2001; Linn, 2000, 2003; Smith \& O'Day, 1991). KERA established ambitious performance standards and improvement targets, ones that the 
creators felt can be reasonably achieved given sufficient effort and supporting resources (Linn, 2003). These definitive accountability guidelines for schools place increased pressure on school leadership to utilize the knowledge base to advance curriculum and instruction.

The Kentucky goals address what leaders need to know and be able to do to support students and teachers in high performing schools (Kentucky Department of Education, 2006b). School leaders have a broad array of responsibilities, none more important in Kentucky's high-stakes accountability arena than the implementation and monitoring of the goals and standards that clearly focus on instructional leadership.

\section{Accountability in Kentucky Education}

When the Commonwealth of Kentucky enacted KERA, the KDE created the Kentucky Instructional Results Information System (KIRIS), a standardized testing tool administered to all students throughout the state each year. In 1998 KIRIS was replaced with a new but more traditional accountability system, the Commonwealth Accountability Testing System (CATS), to improve reliability. The CATS combines criterion-referenced assessment results on the different content areas, along with norm-referenced scores and non-cognitive (e.g., attendance, dropout, and graduation rates) results, into an overall Accountability Index. The index value identifies a school's progress, or lack of, toward the goal of proficiency (school score of 100) by 2014 (Petrosko, 2000). The new assessment system was to build on the strengths of KIRIS while eliminating some significant weaknesses (Poggio, 2000). This index value is given considerable importance toward the reputation of a school as well as an indicator of effective school leadership.

The new CATS system continued the KIRIS system of assessing each student under one of the following standards: (a) novice, (b) apprentice, (c) proficient, or (d) 
distinguished. Each of these standards has a numerical equivalent on a scale of $0-140$ with a school composite score (e.g., Accountability Index) including all students' average scores across all subjects tested. Besides to the academic measures, the accountability index score considers non-academic components, such as "attendance rate, retention rate, dropout rate, and an indicator of successful transition into adult life" (Cobb, 2002, p. 19).

Each school's accountability index score is compared annually to a goal line score to determine if the school is progressing toward its long-term goal. This goal is different for each school dependent upon its own history and demographics. However, all schools through KERA have a common goal of proficiency (100 out of 140) for all students by the year 2014 (Foster, 1999).

The pressure from the high-stakes value-added accountability plays a key role in changing the perspectives on principal leadership in Kentucky. In fact, accountability may be more of a driving force than stated goals or standards (McKinney, 2007). Kentucky's reform expects schools to make continuous improvement toward established goals meaning that principals must continuously seek innovative means of inspiring teachers as well as the educational community to augment learning to increasingly higher levels. Instructional Leadership in Kentucky

The coming together of research findings related to the leadership role of the principal has proven so powerful that state and local practices are becoming shaped by the expectation that principals will act as instructional leaders for their schools (Hallinger \& Murphy, 1986). Such is the case in the Commonwealth of Kentucky's reform initiative of 1990. The Kentucky Education Reform Act firmly placed the responsibility for school improvement on principals. Legislators passed the Effective Instructional Leadership Act (EILA) KRS 156.101 to 
Encourage and require the maintenance and development of effective instructional leadership in the public schools of the commonwealth and to recognize that principals with the assistance of assistant principals, supervisors of instruction, guidance counselors, and directors of special education have the primary responsibility for instructional leadership in the schools to which they are assigned. (KDE, 2004a, p. 4)

Kentucky is one of several states that also adopted the ISLLC standards for school leadership. The ISLLC standards focus on student learning as the measurement of educational leadership success (Lashway, 2002, p. 2). When the school-reform movement began, the first consequence for school leaders was the pressure to put student learning at the center of their jobs simply by paying attention to instruction: setting curricular goals, monitoring lesson plans, and evaluating teachers. In more recent times, instructional leaders engross themselves in the "core technology" of teaching and learning, use data to decide, and align staff development with student learning needs (Lashway).

The Kentucky Department of Education requires that all instructional leadership programs support the following:

1. Kentucky State Board of Education Goals and Objectives;

2. Standards and Indicators for School Improvement;

3. Interstate School Leaders Licensure Consortium (ISLLC) Standards for School Leaders; and

4. Kentucky Department of Education Standards for Professional Development. (KDE, 2004a, p. 6)

Additionally, all leadership programs in Kentucky are, as defined by the technical assistance manual for instructional leaders, to be systemic and continuous on the following topics:

1. making instructional decisions that support teaching and learning;

2. establishing organizational direction; 
3. developing and supporting high performance expectations;

4. creating a learning culture; and,

5. developing leadership capacity. (KDE, 2004a, p. 4)

The emphasis on instruction is hardly misplaced. For all of Kentucky's reforms designed to inspire instructional leadership among administrators, the classroom teacher is perhaps the most significant instructional link (McKinney, 2007). Yet the reality for instruction as suggested by Jackson (1990) is that few recommended reform actions are actually practiced in the schools, particularly so for those that would affect classroom teaching and learning. Morgendollar (1993) argues that change in instructional practices in schools has moved forward far more slowly than activities that are external to actual instruction.

\section{Standards and Indicators for School Improvement}

The executive order that created the Council on School Performance Standards (the Council) was issued in February, 1989, four months before the Kentucky Supreme Court decision was rendered. Thus, the Council had begun some of its initial posturing about what a new education system might look like before the final court order mandating the school reform package that would be passed in 1990. The new reform, the Kentucky Education Reform Act (KERA), included provisions relating to virtually every aspect of education (Willis, Koch, Lampe, Young, Kellor, \& Odden, 1999).

The initial organizational structure of the Council included an executive director, 12 Council members, and five subcommittees. The subcommittees' work was preceded by a survey of 201 Kentucky opinion leaders and 637 members of the general population. The survey content was developed based on the results of focus group interviews with business leaders, parents, educators, employers, and graduates from around the state. About 75 
people worked consistently on the project, increasing to almost 200 as the group worked on defining the new state educational directives (Steffy, 1993). For the task of translating broad capacities and goals into more concrete concepts, the Council used the same processes as it did in the initial stages: the executive director (the same one as hired for the Council's initial work) oversaw 11 task forces. Each task force was chaired by an individual who was recognized and respected as a leader in his or her respective field, and possessed key group leadership and communication skills. The task force members were chosen from more than 450 applicants solicited from throughout the state and included more teachers than any other stakeholder group (Steffy).

Over the first decade of KERA, the focus was on first, implementing the new law in all its many facets. However, an increasing realization occurred by educators of how much greater the focus had to be on improving outcomes for everyone rather than merely accepting achievement based on demographic backgrounds. As the decade progressed, educational leaders recognized more explicitly how difficult school reform was to be. $\mathrm{KDE}$, along with the General Assembly, began the search for a more formal structured process of school improvement, including better diagnostic understanding of whole school reform.

In 1998, the General Assembly passed KRS 158.6455. The intent of this legislation was to ensure that schools succeed with all students and receive appropriate consequences in proportion to that success. Section (3) of the statute charged the Kentucky Board of Education to adopt administrative regulations to establish consequences for schools whose assessment index fell below their assistance line. One possible outcome included a scholastic audit. The Scholastic Audit was designed to examine a school's learning environment, efficiency, and academic performance of students, providing opportunities 
for transforming Kentucky's schools into places where all children can learn at high levels (KDE, 2003). Section (4) of the statute directed the Board of Education to establish guidelines for conducting Scholastic Audits, which included a process for appointing and training teams.

\section{Developing the Standards and Indicators for School Improvement}

Regulations adopted by the Kentucky Board of Education charged Scholastic Audit and Review teams to make recommendations on strategies to improve teaching and learning. This led to the development of the Standards and Indicators for School Improvement, which represented a conceptual breakthrough, i.e., extending the notion of standards-based accountability for curriculum areas to standards for whole school reform. The initial development of the SISI (nine standards and 88 indicators) was completed by the National Study of School Evaluation (NSSE) of Chicago (V. Taylor, personal communication, September 24,2010 ). To validate the use and interpretation of the SISI, NSSE incorporated an encompassing review of extant literature (see Appendix G for the complete list of references used for the development of the SISI document) along with the expert feedback of the Council and General Assembly. Cross team and rater reliability were assessed during audit team training as the teams developed procedures that would remain constant with every completed audit. Triangulated evidence tested assumptions until determinations of performance could be made consistently by the audit teams (Taylor).

Years of comparing the empirical data of high performing and low performing schools provided rudimentary evidence of the external structure validity and fidelity of the instrument (P. Hurt, personal communication, September 22, 2010). However, KDE has done almost no work on validating the SISI and Scholastic Audit derived there from. 
Koger and Thacker (2004) were contracted by KDE to perform a preliminary validation study but this focused more on the process of utilizing the Scholastic Audit than a rigorous assessment of validity.

The Kentucky Department of Education did compare high and low scoring schools across the 88 indicators. KDE (2003) identified indicators in which low performing schools were different from successful schools. These indicators were called Leverage Points in the 2000-2001 samples (see Appendix C) but called Variance Points in the 20022003 samples (see Appendix D). Six Common Variance Points were found in both Leverage and Variance Points in the first two samples (see Appendix E). After the third cycle of audits, KDE identified 11 Common Variance Points (see Appendix F).

Neither KDE nor Koger and Thacker (2004) conducted factor analysis to determine whether the indicators for a given standard held together as a single factor. The series of dissertations on the Scholastic Audit database (B. C. Ennis, 2007; McKinney, 2007; Saravia, 2008) plus work by Smith et al. (2009) do provide this more formal psychometric validation. In this series of studies, the conceptualization of the nine standards and 88 indicators has been confirmed, but only for elementary schools.

In 1990, KERA represented a breakthrough in school reform. KERA presented an educational reform package so sweeping that it completely restructured the state's system of public education. It also garnered the national spotlight of educational reform (Pankratz \& Petrosko, 2000; Steffy, 1993). The SISI document became a part of the KRS 158.6455 statute in 1998 and has remained intact since passage. The behavioral descriptors of the Scholastic Audit are the single portion of the rubric reviewed and altered and then only to ensure clarity for schools as well as audit teams (V. Taylor, personal communication, September 27, 2010). Parallel to the innovations embedded in KERA, Kentucky was the 
first state to develop a model of whole school, the Standards and Indicators for School Improvement and the concomitant Scholastic Audit. Several states have joined the process (adopting or closely adopting the SISI), sending teams to join Kentucky's teams on reviews and audits. Additionally, Kentucky sent audit teams to other states to train and lead them through the audit process (Taylor).

\section{Putting the SISI into Practice}

The Standards and Indicators for School Improvement (SISI) document was adopted by the Kentucky Board of Education as a measurement of a school's readiness for increasing student achievement (KDE, 2004d). Associated documents--District Level Performance Descriptions and Glossary for Kentucky's Standards and Indicators for School Improvement (KDE, 2004a) and a supplementary document for schools, School Level Performance Descriptors for Kentucky's Standards and Indicators for School Improvement (KDE, 2004c)--identify school leaders' responsibilities in three specific areas: (a) Academic Performance, (b) Learning Environment, and (c) Efficiency. Decisions and actions of instructional leadership are anticipated to be in alignment with the competencies outlined in the SISI document.

Standard 7 (Leadership) from the nine standards represents a direct benchmark for Kentucky's principals, the instructional leaders. Consistent with Murphy's (2004) work on principals' influence as indirectly mediated through their efforts in a variety of areas, this study focuses on the areas of curriculum and instruction. Previous work completed on the SISI database was completed for the elementary level (B. C. Ennis, 2007; McKinney, 2007; Savaria, 2008). Of particular interest for this present research is the McKinney study as it looked specifically at Standards 1, 3, and 7. From the perspective of principals, the pursuit of high-stakes accountability goals requires the focus on curriculum and 
instruction. The three relevant standards (KDE, 2004d) are worded as follows:

Standard 1: The school develops and implements a curriculum that is rigorous, intentional, and aligned to state and local standards. (p. 6)

Standard 3: The school's instructional program actively engages all students by using effective, varied, and research-based practices to improve student academic performance standards. (p. 8)

Standard 7: School/district instructional decisions focus on support for teaching and learning, organizational direction, high performance expectations, creating a learning culture, and developing leadership capacity. (p. 20)

A complete list of all nine standards appears in Appendix A. The specific indicators for each of these three standards are listed in Chapter III.

These three standards form the basis of this study's efforts to determine the effect of leadership, more specifically, instructional leadership, on Kentucky performance outcomes as schools strive to achieve educational goals in a high-stakes atmosphere. Standard 7 definitively evaluates school leadership and acknowledges the focus of leadership largely to be placed on the school principal. Principals are legally identified (KDE, 2006b) as the primary responsible part in providing instructional leadership in Kentucky schools. Standards 1 and 3 are designed to advance quality curriculum and instruction. Figure 1 (p. 14) makes explicit that this is a mediated effects model of instructional leadership (Murphy, 2004).

\section{Curriculum, Instruction, and Achievement}

Extensive literature exists that relates both curriculum and instruction to school outcomes, but a far-reaching review is beyond the scope of this study because the primary focus is instructional leadership as mediated by principal activities (Murphy, 2004). A brief yet current examination of research on curriculum and instruction follows. 
From the scholarship reviewed in this study regarding Standard 1 (Curriculum), it is a realistic assumption that under present accountability testing reforms, curriculum choice influences student achievement. However, a more extensive review of extant literature on curriculum is beyond the scope of this study as it is Standard 7 (Leadership) and its mediated effects on curriculum, rather than curriculum itself, that is the focus (Murphy, 2004).

As the instructional leaders of their respective schools, principals can contribute to school success by instituting a high degree of alignment among instructional objectives, curricular materials, and testing instruments (Hallinger \& Murphy, 1986). Numerous studies conducted have revealed a surprising variation across schools in the degree to which students are exposed to the content tested on standardized tests used to assess student achievement and school success (Hallinger \& Murphy, p. 5).

A study conducted by the Horizon Research, Inc. (HRI, 2003) revealed that teachers across the nation representative of typical math and science classrooms cited the most frequent influence on lesson content are state and district standards, followed by the textbook and its program designated for the class, and then state and district accountability systems (Weiss, Pasley, Smith, Banilower, \& Heck). The researchers found that the state and district curriculum standards provide a roadmap for what content teachers teach in 3 out of 4 mathematics and science lessons nationally. The next most common influence on content, the textbook and curriculum programs that are typically selected at the district level, influence 1 out of every 2 teachers' content selections. Lastly, in 1 out of 2 lessons nationally, the content selection is dictated by an accountability system related to student achievement (Weiss et al.).

The instructional leaders, principals, can work to insure that curricular materials 
used in their schools are consistent with the school's instructional objectives, that such materials are mutually reinforcing, and that instructional objectives are aligned with the instruments used to monitor student progress (Hallinger \& Murphy, 1986, p. 5). School leaders need to be aware of the many innovative curriculum strategies that can be employed to help students increase learning, without resorting to narrowing the curriculum. Leaders need to stand firm in the face of intense pressure to raise test scores and remember the importance of maintaining an engaging, comprehensive curriculum (Anthes, 2002).

Schools in Kentucky are expected to develop and implement curricula that are rigorous, intentional, and aligned to state and local standards. Kentucky documents (Academic Expectations, Core Content for Assessment, Transformations, and the Program of Studies) are provided to guide curriculum alignment (KDE, 2004c, p. 5). Expectations for students are set forth as the six learning goals of KERA:

1. Students are able to use basic communication and mathematics skills for purposes and situations they will encounter throughout their lives.

2. Students shall develop their abilities to apply core concepts and principles from mathematics, the sciences, the arts, the humanities, social studies, practical living studies, and vocational studies to what they will encounter throughout their lives.

3. Students shall develop their abilities to become self-sufficient individuals.

4. Students shall develop their abilities to become responsible members of a family, work group, or community, including demonstrating effectiveness in community service.

5. Students shall develop their abilities to think and solve problems in school situations and in a variety of situations they will encounter in life.

6. Students shall develop their abilities to connect and integrate experiences and new knowledge from all subject matter fields with what they have previously learned and build on past learning experiences to acquire new information through various media sources. (KDE, 2007c, p. 4) 
All Kentucky students are expected to achieve the goals as well as the academic expectations. The Program of Studies for Kentucky schools, grades Primary-12, helps ensure all students across the Commonwealth have common content and opportunities to learn at high levels. The school districts and individual schools in Kentucky help students to achieve these goals as they ensure curriculum standards are clearly articulated across all the school levels, $\mathrm{K}-12$. The schools sustain this focal point as they align curriculum between schools that aid students as they transition through the grade levels (McKinney, 2007). Schools, too, are legislated to have a continuing procedure for monitoring, evaluating, and reviewing the curriculum and must provide access to the rigor of the curriculum for all students. Kentucky's Scholastic Audit is unequivocal in identifying a school's effort to ensure the curriculum expectations of Kentucky reform (McKinney). It is worth emphasizing that all of the work and policy directives reviewed in this section are entirely consistent with Murphy's (2004) mediated model of instructional leadership, the theoretical framework that guides this study.

\section{Standard 3, Instruction}

The literature on Instruction (Standard 3) indicates that the cultural and pedagogical strategies employed in the classroom for the presentation of content affect student achievement. More review of extant literature is warranted but beyond the purpose of this paper. Instead, this study's primary focus is Leadership's (Standard 7) mediated effects on instruction (Murphy, 2004).

In planning lessons, teachers are influenced by many factors that determine what content they teach, how they teach it, and what materials they use to engage students with the content (Weiss et al., 2003). The need to understand these contextual influences as precursors to quality instruction must be addressed by instructional leadership for 
increased student achievement to occur (Weiss \& Pasley, 2004). To determine how teachers make instructional decisions and their effectiveness toward quality instruction, HRI conducted a study in 2000-2002 in which its staff and consultants observed a total of 364 math and science lessons in schools across the nation and then conducted extended interviews with the teachers.

For the classroom observations, the researchers used a structured observation protocol, rating each lesson on four components: the lesson design, lesson implementation, mathematics/science content addressed, and classroom culture. The lessons were rated on several indicators within each component area, then provided an overall "capsule" rating (Weiss et al., 2003, p. 1). The researchers found that based on the judgments of the observers, only 15 percent of K-12 mathematics and science lessons would be considered high in quality, 27 percent medium in quality, and 59 percent low in quality. The researchers found that "reform versus traditional" classroom instructional strategies had little bearing on the engagement of students. However, they did surmise that the following factors seemed to distinguish effective lessons from ineffective ones: (a) engage students with the content; (b) create an environment conducive to learning; (c) ensure access for all students; (d) use questioning to monitor and promote understanding; and (e) help students make sense of the content.

Following the classroom observations, extended interviews were conducted with teachers to determine what led them to select the pedagogy and materials used in the lessons (Weiss et al., 2003). The ultimate goal of the interviews was to determine which factors have the greatest influence on the design of the lessons students experience each day. For analysis of the qualitative data, the research team read the observers' descriptions of the lesson designs to determine factors that distinguished designs judged to be effective 
from those judged to be ineffective. The same process was followed for each of the remaining component areas (implementation, content, and classroom culture) and for the final capsule descriptions of entire lessons. In all cases, there was no predetermined coding scheme; rather, the researchers identified themes from the data. The researchers found in 9 out of 10 lessons that teachers had a sense of autonomy in choosing how to implement lessons, that is, teachers had little external guidance in designing their instruction and effective pedagogy. Teachers used resources and strategies grounded in their knowledge, beliefs, and experience, with consideration for the ability levels and behaviors of their students (Weiss et al.).

Implications of both the observation and interview study findings indicate that teachers need a vision of effective instruction to guide the design and implementation of their lessons. Interventions that would likely be helpful to teachers in understanding an overall vision and improving instructional practices include: (a) opportunities are needed to analyze a variety of lessons in relation to key elements of high quality instruction; (b) instructional materials, such as support material accompanying textbooks need to provide more targeted assistance for teachers; (c) teacher development workshops need to reflect the elements of high quality instruction; and (d) administrators need to ensure that teachers are getting a coherent set of messages from state, district, and school levels aligned with one another and in support of the same vision of high quality instruction (Weiss et al., 2003).

In a study by Hallinger, Bickman, \& Davis (1990), an instructional leadership framework (the Far West Lab model) locates the principal's work within both organizational and environmental contexts, thereby reinforcing the idea that the principal shapes the school's instructional climate and instructional organization through interaction 
with teachers and students, as well as development of school policies and norms (p. 5). The model suggests that principals influence student learning by developing a school mission that provides an instructional focus for teachers throughout the school. This creates a school environment that focuses on and facilities student learning (p. 28). Bossert, Dwyer, Rowan, and Lee (1982) expressed the principal's effect on instruction as follows:

Effective principals create the condition...[for successful schooling] by providing coherence to their schools' instruction programs, conceptualizing instructional goals, setting high academic standards, staying informed of policies and teachers' problems, making frequent classroom visits, creating incentives for learning, and maintaining student discipline. (p. 35)

Case studies by Dwyer (1986) found that principals exercise influence on instruction primarily through the development and exercise of "routine behaviors" (Dwyer, Lee, Rowan, \& Bossert, 1983). It is through these routines that the school's instructional climate is shaped.

With the advent of Kentucky educational reform, KDE advocates that schools' instructional decisions be focused on support for teaching and learning, organizational direction, high performance expectations, creating a learning culture, and developing leadership capacity (KDE, 2004c, p. 32). Kentucky school leaders are expected to develop and sustain a shared vision. Leadership decisions are to focus on student achievement. All of the identified foci and activities are consistent with the theoretical framework that guides this study, i.e., Murphy's (2004) mediated effects instructional leadership model. School administration must have a strategic plan focused on effective leadership skills. Schools are expected to disaggregate data for use in meeting the needs of all students, despite ethnicity, and communicate the data to school staff, and ensure the data are incorporated into the school's improvement plan. The instructional programs in Kentucky schools must actively engage all students by using effective, varied, and research-based 
practices to improve student achievement performance (KDE, 2004c, p. 13).

When auditing a school, the audit teams seek for evidence that effective and varied instructional strategies are used in all classrooms. Instructional strategies and learning activities are to be aligned with the state learning goals and assessment expectations for student learning. Instructional strategies are to be aligned for diverse student populations to ensure all learning styles are addressed and that all students have the opportunities to learn at a high level. Again, the principal is held responsible (Standard 7 of the SISI document) for ensuring that this occurs, consistent with Murphy (2004).

Standard 7, Leadership

As processes in educational systems change with time, educators continue to have longstanding appreciation for the necessity of effective leadership in successful schools (Owings, Kaplan, \& Nunnery, 2005). In current educational reforms, credence has been given to the fact that effective school leaders such as superintendents and building level leaders are instructional leaders (Hallinger \& Murphy, 1985). Since the 1980s, the role of building level leaders or principals as instructional leaders has been re-canvassed (Murphy, 2004) as educational reform reconciled inputs and processes to learning outcomes. Principals became responsible for "helping craft a coherent instructional program" (Murphy, p. 66) as well as managing the indirect nature and mediated aspects of instructional leadership (Murphy).

The goals of the current Kentucky reform movement clearly establish high standards for principals with accountability scores determining the point of reference for judging principal performance. Instructional leadership is a key component in Kentucky's reform initiatives. The role of principals as instructional leadership is embedded in the Kentucky legislatively-driven reform initiatives and represent a significant factor in the 
Kentucky audits of school leadership (KDE, 2006b) and are, therefore, consistent with Murphy's (2004) mediated model of instructional leadership.

\section{Kentucky School Audits}

The CATS combines criterion-referenced assessment results on the different content areas (e.g., math, science, English, etc.) along with norm-referenced scores and non-academic results (e.g., attendance, dropout, and graduation rates) into a summary for all students of a school, that is, the Accountability Index. Each school is expected to have an Accountability Index score of 100 by the year 2014, a very high standard (cf. Petrosko, 2000; Rothstein, 2004, chapter 3). The index score of each school is determined by a customized chart that places the school in one of the following categories: (a) meeting goal, (b) progressing, or (c) needing assistance. The schools needing assistance were either required to receive a Scholastic Audit or conduct an internal self-review. The Scholastic Audit, based on the SISI document, was the primary assessment and evaluation instrument for the audits and reviews (KDE, 2003). The goal of the audits was to examine the three areas of the SISI document: Academic Performance, Learning Environment, and Efficiency. The teams trained by KDE consisted of a parent, teacher, school administrator, district administrator, university faculty member, and a Highly Skilled Educator.

The audits provided a glimpse into how schools were progressing toward meeting the goal of proficiency by 2014 . The audit teams, based on observations, interviews, and examination of school documents, rated each of the 88 indicators of the standards of the SISI document. These data points indicated how schools with a low Accountability Index differ from schools with a high Accountability Index.

Audit results are used to help schools and districts accomplish several important goals (KDE, 2003, p. 2): 
1. determine strengthens and weaknesses of a newly implemented program or plan;

2. identify problems early in the implementation process and address them through a continuous improvement model;

3. document early successes as positive feedback to school staff and as supportive evidence for the continuance of the program or plan;

4. enable school staff to base improvement planning on objective data; and

5. formalize school accountability for the success of their comprehensive program or plan.

From the data gathered with the aid of the audits, districts and schools can create, implement, initiate, and monitor programs that will promote the premise that all children can learn at high levels, given adequate time, opportunity, and support.

\section{Summary}

This review of current literature focused on (a) leadership and how perceptions of leadership are changing inclusive of female leadership; (b) educational leadership; (c) instructional leadership; and (d) the accountability and standards movement in education in Kentucky. In the last decade, almost every state in the United States has joined the accountability-based standards movement, which has typically resulted in the implementation of rigorous standards and high-stakes assessment. These processes vary from state to state and continue to change at a fast pace. Kentucky is deeply entrenched in high-stakes accountability as it passed the groundbreaking Kentucky Education Reform Act of 1990 after the present educational system was deemed unconstitutional by the state Supreme Court, due to funding irregularities between school districts.

The Standards and Indicators for School Improvement document is Kentucky's guide for student-centered accountability. The document is divided into nine standards under the three headings of Academic Performance (Standards 1, 2, \& 3), Learning 
Environment (Standards 4, 5, \& 6), and Efficiency (Standards 7, 8, \& 9). The standards constitute the cumulative effect for whole school improvement and high student academic achievement (KDE, 2003, p. 10). Along with the nine standards are five to sixteen indicators for each standard ( 88 total) which detail school expectations for achieving student success. To evaluate the effectiveness of school planning and strategic implementation of the standards, each indicator was defined by a 4-point behavioral rubric that became known as the Kentucky Scholastic Audit.

In Kentucky, instructional leadership has been redefined by the Standards and Indicators for School Improvement document, placing the pivotal responsibilities of student success in the hands of the building level administrators--the principals. The dissemination of effective schools research of the 1980s concurred with the expectation that the principal has a definitive impact on student academic achievement. This is consistent with the theoretical framework that guides this study, Murphy's (2004) mediated instructional effects model. Using the data collected through the Scholastic Audits, it is the goal of this study to address the central research question: Based on Scholastic Audits, what are the effects of Leadership, Curriculum, and Instruction on Kentucky accountability scores? 


\section{CHAPTER III}

\section{METHODOLOGY}

\section{Type of Research}

This quantitative research study analyzed secondary data by the Kentucky Department of Education (KDE): (a) to examine the effects of school level leadership on curriculum and instruction as they pertain to student success depicted by the Academic Index scores in Kentucky high schools, and (b) to investigate relationships, if any, that may exist among certain demographic factors such as school size, school setting, gender, and student achievement. The data were compiled through Scholastic Audits of both successful and struggling schools in Kentucky. The Scholastic Audit is the process used by a KDEtrained team to provide an intense review of academic effectiveness and learning environment of schools to determine why they are not meeting their established base line goals. The goal of each Kentucky school is a score of 100 out of 140 on the Accountability Index by the year 2014 .

The primary form of analysis is multiple regressions. In regression, an equation with several independent variables is estimated in an attempt to isolate the separate effect each has on the dependent variable (Smith, 1985, p. 515). The school was the unit of analysis for both collection of data by KDE and for this study with the objective of determining the relationships among leadership, curriculum, instruction, and accountability scores, along with demographic factors. Research indicates that socioeconomic factors in the schools and community influence principal leadership and its effect on school success 
(e.g., Andrews \& Soder, 1987; Hallinger \& Murphy, 1986). This work replicates a study by McKinney (2007) who examined elementary level Scholastic Audits whereas the current effort utilizes data from high schools. This investigation is the first to analyze Kentucky Scholastic Audits for high schools.

The Academic Index was identified as the dependent variable. Three of the nine standards from the Standards and Indicators for School Improvement (SISI) document (KDE, 2004a) have been designated as independent variables: Leadership, Curriculum, and Instruction. Demographic influences were identified as control variables (McKinney, 2007). Scores for the Leadership, Curriculum, and Instruction standards were obtained from the Scholastic Audits while the dependent variable and demographic indicators were obtained from the Kentucky Performance Report (KPR).

Leadership, in particular, is closely connected to the two other standards. Wagner (1998) states that the principal's activities-deciding on what should be taught in schools (curriculum) and how it should be taught (instruction)--are important criteria surrounding school success. In addition, other influences are weighing on accountability scores over which this researcher has no control; however, with the combination of factors included in this study, this analysis yields useful statistical data for guiding leadership development and reform initiatives.

Definition of Terms for Kentucky's Accountability System Academic Index for Secondary Schools (AI): The Academic Index comprises $90.5 \%$ of the overall Accountability Index. Academic content values include: Reading, 14.25\%; Mathematics, 14.25\%; Science, 14.25\%; Social Studies, 14.25\%; On Demand Writing, 2.85\%; Writing Portfolio, 11.4\%; Arts and Humanities, 7.125\%; Practical Living Nocational Studies, 7.125\%, and NRT, 5\% (KDE, 2005a, p. 71). 
Assistance Line: "Represents the point below which a school becomes eligible for assistance from the state. A straight line that begins at the baseline in 2002 and ends at 80 in 2014 represents this line"(KDE, 2005a, p. 2).

Baseline Accountability Index: The Accountability Index score was determined by averaging the performance during the 1998-1999 and 1999-2000 school years. This is the number against which progress is measured each biennium (KDE, 2005a, p. 2). For this study, not all Scholastic Audits were conducted in the same year. If the 1998-2000 Baseline Accountability Index was not appropriate in a particular case, the most recent baseline before when the audit was conducted was utilized.

Commonwealth Accountability Testing System (CATS): In 1998 CATS replaced KIRIS that was the previous accountability system. The CATS has a higher reliability with less emphasis on performance assessment (KDE, 2005a). The fundamental high-stakes, value-added nature of the accountability system, however, remained the same. The Academic Index goal for every school in Kentucky is Proficiency as defined by the Kentucky Board of Education (KBE). The goal of Proficiency translates into a school Accountability Index value of 100 . More explicitly, the goal of the KBE for each school is to achieve an Accountability Index no less than 100 by 2014. The major characteristics of the accountability model are that it involves (a) an index, (b) a measure of growth between successive cohorts (groups of students at the same grade, but in different years), (c) criteria that are applicable to the whole school, (d) differential weighting of indicators, and (e) recognition points--an indicator of absolute standing against Kentucky's performance standards (KDE, 2004b, p. 13).

Goal Line: "Represents the point above which schools are identified as successful schools. A straight line represents where it began in 2000 and will end in 2014" (KDE, 
2005a, p. 2).

Highly Skilled Educator (HSE): Persons identified and hired by KDE who are charged with improving student achievement in low performing schools. These persons are extensively trained by KDE in procedures for school assistance and in the audit process as framed by Kentucky's SISI document (KDE, 2006c).

Kentucky Core Content Tests (KCCT): The criterion-referenced tests taken by all Kentucky students in Grades 4, 5, 7, 8, 10,11, and 12 administered in the Spring of each school year. In Grades 4 and 7 students are tested in reading and science. Grade 5 and 8 students are tested in mathematics, social studies, arts \& humanities, and practical livinglvocational studies. Reading and practical livinglvocation studies are tested in Grade 10. Students in Grade 11 are tested in mathematics, science, social studies, and arts \& humanities. Grade 12 students have a test of two writing prompts (questions), selecting one of the two. All other grades, except Grade 12, have both open-response and multiplechoice questions. Grade 7 also has two writing prompts like grade 12 (KDE, 2005a, p. 65).

Kentucky Instructional Results Information System (KIRIS): The testing system used in Kentucky for accountability assessment from 1991-1998. It was replaced by CATS in $1998(\mathrm{KDE}, 2005 \mathrm{a})$.

Kentucky Performance Report (KPR): The reports give detailed information on the results provided by the Kentucky Core Content Tests (KCCT), Writing Portfolio, NormReferenced Test, and other components of the Commonwealth Accountability Testing System (CATS). As required by KRS stature, these reports are received by school districts on or before September 15th of each year. Data in the reports are constructed from information provided by many sources: testing consultants, students, schools, district offices, and KDE. The KPR is designed to show performance for all student 
subpopulations and content areas at the elementary, middle, and high school levels. Thus, most school and all district KPRs contain data from at least two grades levels (e.g., grades 10,11 , and 12 at the high school level). The assessment and accountability results are the most important part of the KPR, providing accountability classification summary information for the school including the school's growth chart (KDE, 2006e).

Leverage Point: An indicator of disproportional results from successful to Level 3 Assistance Schools as determined by the findings of the 2000-2001 round of Scholastic Audits (Kentucky Department of Education, 2003). Of the nine 88 Indicators (across the nine Standards), 17 were designated as Leverage Points (KDE, 2003).

Mediated: The indirect influence of the principal on student success through efforts in curriculum and instruction (Murphy, 2004).

NAPD Descriptions: This denotes Novice, Apprentice, Proficient, and Distinguished--the categories used to report student results on the CATS. Each classification is assigned a numerical equivalent on a scale of 0-140 (KDE, 2005a, p. 65).

The following are the descriptions for the four levels:

1. Distinguished: Student demonstrates an in-depth, extensive, or comprehensive knowledge of content. Student communication is complex, concise, and sophisticated with thorough support, explicit examples, evaluations, and justifications. Student uses and consistently implements a variety of appropriate strategies. Student demonstrates insightful connections and reasoning.

2. Proficient: Student demonstrates broad content knowledge and is able to apply it. Student communication is accurate, clear, and organized with relevant details and evidence. Student uses appropriate strategies to solve problems and make decisions. Student demonstrates effective use of critical thinking skills.

3. Apprentice: Student demonstrates some basic content knowledge and reasoning ability. Student communicates reasonably well but draws weak conclusions or only partially solves or describes. Student attempts appropriate strategies with limited success.

4. Novice: Student demonstrates minimal, limited, underdeveloped, and at times 
inaccurate content knowledge and reasoning. Student communications is ineffective and lacks detail with no evidence of connections within or between content areas. Student uses inappropriate strategies. (KDE, 2005a, pp. 65-66)

Norm Referenced Test (NRT): The NRT is based on the Comprehensive Test of Basic Skills (CTBS5) Survey in reading, language arts, and mathematics. This test is given so Kentucky students can be compared with students nationally. The "index" for this test is an average of student scores weighted according to the national percentile range as follows:

$\begin{array}{cc}\text { Weight } & \text { National Percentile Range } \\ 0 & 1-24 \\ 60 & 25-49 \\ 100 & 50-74 \\ 140 & 75-99\end{array}$

Assigning the above weights places the NRT on the same $0-140$ scale as the academic areas on the core content tests. The NRT is $5 \%$ of the Accountability Index for school levels--elementary, middle, and high (KDE, 2005a, pp. 70-71).

Nonacademic Index: This index represents $9.50 \%$ (attendance, 1.90\%; dropout, $3.56 \%$, retention, $0.48 \%$, and successful transition to adult life, $3.56 \%$ ) of the nonachievement factors of the overall Accountability Index for high schools (KDE, 2005a, $p$ 71).

Scholastic Audit: By virtue of Section 4 of the Kentucky Revised Statues (KRS) (158.6455), guidelines were established to review a school's learning environment, efficiency, and academic performance of students; evaluate each certified staff member assigned to the school; and, report to the KBE about the school's classification and what 
would be necessary to improve teaching and learning in the school being audited.

Categories established for the Scholastic Audit process include:

1. Successful School: Schools that had an Accountability Index score on the CATS that met or exceeded their biennium goal. (KDE, 2003, p. 7)

2. Assistance School: All schools with scores that placed them below the assistance line. There are three levels:

a. Level 1 Assistance School--an Accountability Index score on the CATS that puts it in the upper one-third of schools below their assistance line. The majority of these schools complete a Self-Review using the SISI document as a guide. (KDE, 2003, p. 9)

b. Level 2 Assistance School--an Accountability Index score on the CATS that puts it in the middle one-third of schools below their assistance line. A Scholastic Audit is completed at these schools. (KDE, p. 9)

c. Level 3 Assistance School--an Accountability Index score on the CATS that puts it in the lower one-third of schools below their assistance line. These schools are required to have a Scholastic Audit. (KDE, p. 7)

Scholastic Review: The same audit procedure is used as in the Scholastic Audit but the audit team is changed to two representatives from the regional service center and two to four representatives from the school's district (KDE, 2003).

Standards and Indicators for School Improvement: A set of nine standards with 88 indicators determined by $\mathrm{KDE}$ to represent whole school reform. Assessments of these standards and indicators form the basis of recommendations for assistance to schools (KDE, 2004d).

Successful School: A school was designated as successful if the CATS accountability index was at, or above, its goal for the biennium (KDE, 2003).

Variance Point: After the Scholastic Audits were completed in 2002-2003, the term Variance Point replaced the term Leverage Point to reflect more appropriately the meaning of the term. A total of 27 indicators were identified as Variance Points from that round of 
audits (see Appendix D). Six Common Variance Points (Appendix E) existed when the two rounds of the Scholastic Audits were examined, 2000-2001 compared with 2002-2003 (KDE, 2003). After the third round of audits (2004-2005), KDE found 11 Common Variance Points across the entire set of data (see Appendix F). After the third round, KDE used revised criteria on what constituted significant variance resulting in the reduction of Common Variance Points from 27 to 11 (KDE, 2006f).

KDE established these Leverage and Variance Points based upon the entire set of completed audits, which includes elementary, middle, and high schools. KDE has not disaggregated those levels of schools in any of the analyses they have conducted. In contrast, the current study utilizes high schools only while the completed dissertations by B. C. Ennis (2007), McKinney (2007), and Saravia (2008) were based on elementary schools.

\section{Description of the Data}

The data utilized for this study were collected by KDE-trained audit teams via scholastic audits or reviews performed using Kentucky's Standards and Indicators for School Improvement document as the primary assessment and evaluation instrument. One hundred thirty-one schools were either audited or reviewed during the 2000-2001 school year. As reported by KDE, the 2000-2001 audits were divided as follows: 83 elementary schools, 28 middle schools, and 20 high schools. Based on the Accountability Indices of the schools, the following levels were established: 47 Level 3 schools (Scholastic Audit required), 50 Level 2 schools (Scholastic Review required), 15 Level 1 schools (Voluntary Scholastic Reviews conducted), and 18 successful schools (Scholastic Reviews were conducted in a sample of successful schools). Classifications for the 114 schools audited during the 2002-2003 are: 29 Level 3 schools, 32 Level 2 schools, 29 
Level 1 schools, and 24 successful schools (KDE, 2003). The 2004-2005 audits resulted in 48 schools being classified as in need of assistance (KDE, 2004d). This study has limited its data to Kentucky high schools that completed a Scholastic Audit or Scholastic Review from 2000-2005. This includes $N=83$, all of the 224 high schools that contain Grades 9 through 12. The NRT Index comes from Grade 9, but the NRT is not part of the Academic Index.

This study does not measure the progress of the same students each year and therefore is not longitudinal. It follows a cohort model where different students are assessed each year of the accountability cycle or biennium (B.C. Ennis, 2007).

The study relies on the results of the KDE audit team findings and their use of consistent audit training and processes. Thus, the research is dependent upon the reliability and validity of Kentucky's audit and testing processes.

The Accountability Process

"The purpose of the Commonwealth Accountability Testing System (CATS) is to encourage and enable educators in each public school to increase the academic achievement of their students" (KDE, 2005a, p. 1) and to determine if the academic goals of schools are being met. The system assesses each student with the criterion-referenced Kentucky Core Content Tests (KCCT) and the norm-referenced Comprehensive Test of Basic Skills (CTBS). Results for each student are evaluated at one of the following levels: (a) novice, (b) apprentice, (c) proficient, or (d) distinguished. Each of these standards has a numerical equivalent on a scale of 1-140. A school's composite of the average of students' scores across all subjects tested is the Academic Index (AI). This AI, coupled with nonacademic indicators such as attendance and retention, determine the school's Accountability Index. 
The Accountability Index measures whether or not each individual school's longterm goal of increased student achievement is being met. The annual index for each school is determined based on its own history, demographics, and original baseline score. The goal of the KDE is that each school reaches the Proficiency level, that is, 100 on a 140point scale, by the year 2014. The KDE divided the period of 2000-2014 into seven, 2-year accountability cycles to establish parameters for improvement. Every two years, schools are assigned levels based on their Growth Accountability Index scores determined by averaging the Accountability Index for each cycle. Simply stated, each year schools receive an Accountability Index score but every two years, they receive a Growth Accountability Index score that is an average of the two previous years. It is this score that determines the level a school will be assigned. Schools can be assigned Level 1, 2, or 3, as defined in Definition of Terms above. If a school receives a classification of Level 3 for two or more consecutive accountability cycles, the school is required to have a Scholastic Audit conducted by KDE. If the school is determined to be a Level 2 school, a Scholastic Review is conducted; and, if the school is Level 1, it is classified a successful school with no audit or review required. The Standards and Indicators for School Improvement: Kentucky's Model for Whole School Improvement (KDE, 2003) is the comprehensive framework used by the audit teams to determine the areas of needed improvement and to make the appropriate recommendations to help the school(s) succeed in respective improvement efforts.

\section{Scholastic Audit Methodology}

The Division of School Improvement (Office of Leadership and School Improvement) of the Kentucky Department of Education was instrumentally responsible for establishing the procedural processes and conducting the training for the audit and 
review teams. The audit teams consisted of a parent, teacher, school administrator, district administrator, university faculty member, and a Highly Skilled Educator (HSE). The review teams consisted of two regional service center representatives and two to four school district office representatives (KDE, 2003).

The SISI document was the primary assessment and evaluation instrument (KDE, 2003, p. 9); however, the audit and review teams also gathered information from (a) leadership and school culture surveys, (b) examination of documents in the school portfolio, (c) team experiences, (d) interviews, and (e) observations. A school's portfolio included a variety of materials and documents pertinent to the school, including the Comprehensive School Improvement Plan, state assessment results, student achievement data, non-academic data, writing portfolio analysis data, school survey data, district technology inventory, school handbook and master schedule, school report card, SchoolBased Decision Making Council policies and meeting minutes, teacher unit lesson plans, district evaluation plan, curriculum documents, examples of student work, and listings of school professional development activities (p. 9). The school profile was fashioned through the analysis of (a) the portfolio information, (b) classroom observations, and (c) formal and informal interviews with teachers, students, parents, the principal, assistant principals, counselors, and central office staff. The audit and review teams evaluated the collected evidence by comparing it with the Performance Descriptors for Kentucky's SISI document for each indicator, under each standard, and then by agreement established a score for each indicator. The following scale was used:

Category 1--Little or no development implementation

Category 2--Limited development and partial implementation Category 3--Fully functioning and operational level of development and 
implementation

Category 4--Exemplary level of development and implementation. (KDE, 2004c)

For the purposes of this study the audit scores of 1-4 were converted to scores of 0 , 2, 5, and 7 (see McKinney, 2007). This represented a more realistic view of the difficult move from limited development (2) to fully functioning (3) and parallels the larger gap in Kentucky's scoring rubric associated with moving students from Apprentice to Proficient on the four criterion levels of achievement for the 140-point KCCT scale (Novice, 0-39; Apprentice, 40-99; Proficient, 100-139; and Distinguished, 140).

Based on assimilated data, the audit and review teams offered recommendations and next steps for each standard. When indicators were evaluated as exemplary, commendations were written. The findings of the reports were organized into nine standards under the headings of Academic Performance (Standards 1, 2, and 3), Learning Environment (Standards 4, 5, and 6), and Efficiency (Standards 7, 8, and 9). (See Appendix B for the nine standards.) The resulting school profile indicated a comprehensive view of the nine standards with their supporting indicators. Although KDE emphasizes the cumulative effect of all the standards with respect to whole-school improvement (Kentucky Department of Education, 2003, p. 10) such as with the audits and reviews, the examination of the nine standards is beyond the scope of the current study. Only the scores given to the indicators for Standard 7 (Leadership), Standard 1 (Curriculum), and Standard 3 (Instruction) are used as data for this current study. Notwithstanding, the extensive data collected in the Scholastic Audits and Reviews represent an invaluable resource for future researchers.

\section{Description of the Variables}

The Scholastic Audit was the primary source of data for this study, for Standards 1, 
3, and 7. Additional data were collected from the Kentucky Performance Report (KPR), a database maintained by KDE on individual schools. Academic Index and selected demographic variables were taken from this source. Data from these two separate sources were merged into a single EXCEL file and transported into SPSS for analysis.

\section{Dependent Variable}

The dependent variable for this study is the Academic Index (AI) score, as defined in the Definition of Terms listed above. The Academic Index scores were obtained from the Kentucky Performance Report (KPR) and constitute most of the overall Accountability Index. The AI is a school level aggregation of the student level scores as weighted by the different content areas.

\section{Independent Variables}

For this study there are three types of independent variables: (a) Control, (b) Leadership, and (c) Mediating Factors, arranged consistent with the designations in Figure 1. The Control factors constitute Bloom's (1980) non-alterable, socio-demographic background. The list of Control factors reflects prior work by B. C. Ennis (2007), McKinney (2007), and Saravia (2008) on a larger set of demographic factors. Collectively that set of studies established that only six of these factors had an impact on either the Academic Index or the different standards (see B. C. Ennis). The current study examines the larger, more comprehensive set of variables because no previous work has been conducted for high schools.

\section{Control Variables}

The research is designed to control for demographic factors within a school that are likely to affect student achievement thus school achievement. Common demographic factors included in this study are listed in Figure 1. 
Appalachian Status (APP): The Appalachian region in Kentucky is an area where the influence of mining typically reinforces the traditional rural mindset (Caudill, 1963). Close to a third of Kentucky's students are in counties defined as Appalachian (Smith, $2005,2006)$. The dichotomous variable is coded $1=$ Appalachian region, $0=$ not Appalachian region.

County/Independent Schools (CTY): Schools situated within County systems/districts as opposed to independent schools/districts), dichotomously coded $1=$ County, $0=$ Independent.

Percentage Disabled (\%DIS): The percentage of students in grades 9-12 receiving special education services within each school as tallied in the KPR (ratio measure). This is a continuous variable running from 1-100.

Percentage Free Lunch Participation (\%FRL): Free and reduced lunch information as a proxy for socioeconomic status (SES) is a familiar practice in education. Although FRL is not a good measure of SES (an under estimate of true effect of stratification), feasibility makes this the variable of choice in many studies (Harwell \& LeBeau, 2010). Individual schools report this information to the KPR; ratio measure is coded as a percentage for free eligibility and is a continuous variable running from 1-100.

Percentage GiftedTalented (\%GFT): The percentage of students (ratio scale and a continuous variable running from 1-100) who have been identified by the individual schools to participate in gifted and talented programs as reported in the KPR.

Percentage Limited English Proficient (\%LEP): Ratio measure, the percentage of students identified as speaking English as a secondary language as reported by the KPR. This is a continuous variable running from 1-100.

Percentage White (\%WH): Race and ethnicity differences are measured by the 
percentage (ratio) of majority students (White) in grades 9-12 for each student as tallied for individual schools in the KPR, based on student coding of $1=$ White, $0=$ all other. This is a continuous variable running from 1-100.

School Size (SIZE): Ratio measurement of number of students enrolled in grades 10 -11 , obtained from the Kentucky Performance Report. This is a continuous variable running from 1-100.

Year of Audit (YR): This variable represents the year in which the Scholastic Audit was conducted, coded as an interval variable from 2001 to 2005. KDE has established a timeline of 2014 for all schools to reach proficiency, thus establishing each year as a measure of progress toward that goal. This is an ordinal variable. Efficiency

The guide for school improvement in Kentucky--The Standards and Indicators for School Improvement document--is based on extensive research and consultation by the Office of School Improvement and defines the elements of whole-school improvement at every school level, elementary, middle, and high (KDE, 2004b, p. 176). It is framed by nine standards under the headings of Academic Performance (Standards 1, 2, and 3-Curriculum; Classroom Evaluation/Assessment; and Instruction), Learning Environment (Standards 4, 5, and 6--School Culture; Student, Family and Community Support; and Professional Growth and Evaluation), and Efficiency (Standards 7, 8, and 9--Leadership; Organizational Structure and Resources; and Comprehensive and Effective Planning). The SISI document serves as the evaluative tool for Kentucky schools. These guidelines constitute the elements of whole school reform that are to lead to effective schools.

In this study, the alterable variable under Efficiency is Leadership (Standard 7 of the SISI document). It definitively states that schoo/district instructional decisions focus 
on support for teaching and learning, organizational direction, high performance expectations, creating a learning culture, and developing leadership capacity (KDE, 2003, p. 21). Of the 88 total indicators, eleven of them evaluate school leadership in terms of Kentucky's goals and standards for school leaders, inclusive of alliance with the ISLLC standards as previously discussed. The indicators for leadership are as follows:

Leadership (LEAD)

a. Leadership has developed and sustained a shared vision.

b. Leadership decisions are focused on student academic performance and are data-driven and collaborative.

c. There is evidence that all administrators have a growth plan focused on the development of effective leadership skills.

d. There is evidence that the schoo/district leadership team disaggregates data for use in meeting the needs of a diverse population, communicates the information to school staff and incorporates the data systematically into the school's plan (CSIP).

e. Leadership ensures all instructional staff has access to curriculum related materials and the training necessary to use curricular and data resources relating to the learning goals for Kentucky public schools.

f. Leadership ensures that time is protected and allocated to focus on curricular and instructional time.

g. Leadership plans and allocates resources, monitors progress, provides organizational infrastructure, and removes barriers in order to sustain continuous school improvement.

h. The schoo/district leadership provides the organizational policy and resource infrastructure necessary for the implementation and maintenance of a safe and effective learning environment.

i. Leadership provides a process for the development and the implementation of council policy based on anticipated needs.

j. There is evidence that the SBDM (Site Based Decision Making) council has an intentional focus on student academic performance. 
k. There is evidence that the principal demonstrates leadership skills in the areas of academic performance, learning environment, and efficiency. (KDE, 2004b, pp. 32-37)

\section{Mediating Factors}

In this model (see Figure 1) Standards 1 and 3--Curriculum and Instruction--are the direct influences on the classroom. These two respective standards represent what the students are to learn (the content) and how the teachers are to present the material (instruction). Each has an ongoing and immediate impact on student learning. In this study, it is presumed that they mediate the indirect effect of Standard 7, Leadership.

Curriculum, Standard 1 of the SISI, directs schools to develop and implement a curriculum that is rigorous, intentional, and aligned to state and local standards. For example, Curriculum Indicator 7.e. requires principals to ensure all instructional staff has access to curriculum related materials and the training necessary to use curricular and data resources relating to the learning goals for public schools in the state of Kentucky. Seven of the indicators evaluate curriculum in terms of Kentucky goals and standards for school leaders:

\section{Curriculum (CURR)}

a. There is evidence that the curriculum is aligned with the Academic Expectations, Core Content for Assessment, Transformations and the Program of Studies.

b. The district initiates and facilitates discussions among schools regarding curriculum standards to ensure they are clearly articulated across all levels (P-12).

c. The district initiates and facilitates discussions between schools in the district in order to eliminate unnecessary overlaps and close gaps.

d. There is evidence of vertical communication with an intentional focus on key curriculum transition points within grade configurations (e.g., from primary to middle and middle to high). 
e. The school curriculum provides specific links to continuing education, life, and career options.

f. There is in place a systematic process for monitoring, evaluating, and reviewing the curriculum.

g. The curriculum provides access to a common academic core for all students. (KDE, 2004b, pp. 5-8)

Instruction, Standard 3 of the SISI, directs schools to engage all students actively by using instructional practices that are effective, varied, and research-based to improve student academic performance. As an example, Leadership Indicator 7.f. requires principals to ensure that time is protected and allocated to focus on curricular and instructional issues. Eight indicators evaluate instruction in terms of Kentucky goals and standards for school leaders:

Instruction (INST)

a. There is evidence that effective and varied instructional strategies are used in all classrooms.

b. Instructional strategies and learning activities are aligned with the district, school, and state learning goals and assessment expectations for student learning.

c. Instructional strategies/activities are consistently monitored and aligned with the changing needs of a diverse student population to ensure various learning approaches and learning styles are addressed.

d. Teachers demonstrate the content knowledge necessary to challenge and motivate students to high levels of learning.

e. There is evidence that teachers incorporate the use of technology in their classrooms.

f. Instructional resources (textbooks, supplemental reading, technology) are sufficient to effectively deliver the curriculum.

g. Teachers examine and discuss student work collaboratively and use this information to inform their practice.

h. There is evidence that homework is frequent and monitored and tied to 
instructional practice. (KDE, 2004b, pp. 13-16)

Analysis Plan

The various facets of data analysis used in this study are discussed in the next sections. Data analysis includes: data checking/coding, descriptive statistics, psychometric analysis, and multiple regression.

\section{Data Checking and Coding}

Vogt (2005) describes secondary data as "information collected by other researchers but available sometimes in databases, for use by others" (p. 290). This study is based on data collected by the Kentucky Department of Education or its representatives. Rosenberg, Greenfield, and Dimick (n.d., p. 14) discuss several advantages for using secondary data: (a) the availability of the data, (b) less expense to obtain or to access, (c) the ability to investigate research questions by using large populations or samples, (d) the avoidance of selection bias inherent when using primary data, and (e) the ease of human subjects and administrative requisites, such as the informed consent, which have been taken care of in the stage of primary data collection. Using secondary data also allows performing a series of examinations to see if any of the variables violate major assumptions of regression, i.e., linearity, reliability of measurement, homoscedasticity, and normality of the data.

There can also be limitations on the use of secondary data. Nachmias and Nachmias (1987) discussed the disadvantages of using secondary data: (a) the data may not be exactly the type of data the researchers would like to have for testing hypotheses, (b) the description of how the data were collected may be limited, and (c) there may be a gap between data collected by the investigator with specific research purposes and intentions and the data collected by someone else. 
These drawbacks are not germane to this study as the data base is limited to schools participating in the audit process, and the audits constitute the measurement of the constructs being investigated. By Kentucky law, every school has to participate in CATS and would have a KPR. Therefore, missing data would be minimal. Each school was responsible for reporting all demographic data. Although data are blocked on the KPR so that individual students cannot be identified, the summary data would be complete and combined into a single data set. While this offers valuable insight when low-performing schools are compared with successful schools, the audit data are not equally divided between low-performing schools and voluntary school settings, and the circumstances surrounding the audit are somewhat different.

Admittedly, reliability and accuracy of these data are dependent upon the care with which they are compiled. The database used was compiled by the Kentucky Department of Education by highly-trained auditors and clearly defined audit documents. Kentucky's audit procedure offers the researcher the benefit of a thorough, on-site survey of conditions at the school level, framed by the state's accountability model. Questions about authenticity and accuracy related to the Scholastic Audit data used in this research are minimized by using trained audit teams and consistent audit procedures (Koger \& Thacker, 2004). Additionally, the use of data gathered by an intense, thorough, in-school, and inthe-classroom audit is an opportunity rarely afforded educational researchers (McKinney, 2007).

\section{Descriptive Analysis}

Descriptive statistics are reported for both the independent variables and the dependent variables (the Academic Index). The independent variables are organized according to the three types of predictors (columns) described in the theoretical model 
(Figure 1). In this section, the demographic factors and dependent variable are reported. Descriptives for the Leadership, Curriculum, and Instruction standards are included in the section below.

\section{Psychometric Analysis}

Psychometric analysis involves the application of factor analysis and Cronbach's (1951) alpha. These results determine whether the variables can be conceptually and statistically related or grouped together, thus determining whether data reduction is possible. Since the audit process yields a numeric value for each indicator for each standard, the Scholastic Audit does not provide an overall value for each standard. The question therefore arises whether a single score for each standard is viable. Factor analysis provides a means to resolve that issue by ascertaining which individual variables load together on a single factor based on the interrelationships among the items (the indicators), with the new factor treated as a single variable. Factor analysis is thus performed by "finding patterns among the variations in the values of several variables" (Vogt, 1999, p. 108). In this study, a separate factor analysis was conducted on the set of indicators for each of the three standards (Leadership, Curriculum, and Instruction).

It is possible that a given variable, which loads with others on a factor derived from the factor analysis, can have a poor reliability with the other indicators in that new factor. Cronbach's alpha is conducted to determine the internal reliability of these factors. For this study, Cronbach's alpha indicated the internal consistency of the set of factors derived from the factor analysis of the standards for Leadership, Curriculum, and Instruction. A high alpha value suggests indicative of variables measuring the same construct; that is, there is good internal reliability of the scale. A low alpha value implies that the variables are measuring multidimensional constructs, suggesting that the items are best viewed as 
separate variables. Cronbach's alpha ranges from 0 to 1.0 ; Vogt $(1999, \mathrm{p} .64)$ suggests that scores at the high end of the range, 0.7 or above, indicate that the items have an acceptable level of consistency.

\section{Regression Model}

Multiple regression was created for cases involving two or more independent variables in which the research is designed either to predict or to explain the behavior or a single dependent variable. According to Huck (2000) this kind of analysis is appropriate when there are two or more independent variables but only one dependent variable being analyzed. This method can also determine the degree to which variables are related, as well as an estimate of the overall effect size. In this research, multiple regression is used to determine the extent to which the variability of the Academic Index scores (the dependent variable) could be explained by the different independent variables--leadership, curriculum, instruction, and demographic factors. Multiple regression also has a number of assumptions regarding the type and shape of the data, relationships among variables, etc. The researcher is responsible for checking to see that these assumptions have been met.

While this research tests theoretical assumptions about school leadership, curriculum, and instruction, it also examines their influence on accountability scores. The comparative significance of each predictor may be determined based on the degree to which predictor variables account for variance in the Academic Index scores. The focus is therefore not on determining the most advantageous set of independent variables but rather to investigate the extent that Kentucky goals for leadership, curriculum, and instruction influence school success. Multiple regression allows the researcher to accommodate variables that are to be controlled, in this case the demographic factors. For this study, the control variables examined are well documented within the educational arena as influential 
in student educational success. These factors also parallel the demographic factors examined for their influence in the three completed dissertations on the Scholastic Audit at the elementary level (B. C. Ennis, 2007; McKinney, 2007; Saravia, 2008). Because Kentucky goals and objectives, as encapsulated in SISI, presume a strong link among leadership, curriculum, instruction, and school success, the statistical results of this study represent an estimate of the efficacy of these guidelines for the support needed by schools toward continued improvement.

The mechanics for computing multiple regression estimates are mathematically complicated. Statistical software (SPSS) is used to calculate results. As anticipated by the researcher and as prior Kentucky accountability investigations have shown, the independent variables utilized in this study are likely to have substantial, statistically significant effects on a school's Academic Index based on elementary level work (B. C. Ennis, 2007; McKinney, 2007; Saravia, 2008). Although other external variables and organizational structures specific to individual schools represent a challenge for determining causal relationships (Hallinger \& Heck, 1998), this study attempts to measure the degree to which principals in Kentucky public schools contribute to school success.

At this juncture, worthy of note is the selection process for schools in the research data since multiple regression analyses of sample populations infer results to entire populations. Random samples of Kentucky schools are not administered the Scholastic Audit; rather, schools included in this study data are both low-performing schools (Audits' Reviews mandatory) and successful schools (Audits/Reviews voluntary). Although the audit procedures are similar for all schools, the reason for the audits is different. In lowperforming schools, school personnel may view the audits as threatening and thus attitudes may be more defensive. In successful schools, the audit is more likely to be viewed as 
useful for gaining positive recognition or data for further improvement. Such attitudes may influence auditor perceptions to a degree and limit generalizability (McKinney, 2007). Further, the sample is select, i.e., high or low performing. The greater number of schools in the middle (progressing as expected, not falling behind or doing better), are generally not a part of such atypical samples.

\section{The Multiple Regression Formula}

The simple regression formula, $\mathrm{y}^{\prime}=\mathrm{a}+\mathrm{bx}$, shows how an independent variable is related to a dependent variable where $y^{\prime}$ represents the dependent variable, $a$ is the $y-$ intercept (the value of $y$ if the dependent variable was zero), and $b$ gives the estimated effect on the dependent variable (y) for each unit change in the independent variable. Since this research has multiple independent variables, the appropriate formula would be $y^{\prime}=a+$ $b_{1} x_{1}+b_{2} x_{2}+\ldots+b_{n} x_{n}($ Bernard, 2000). All subsequent regressions are based upon the Pearson $r$ full correlation matrix included in this section, provided the variables are continuous.

\section{Multiple Regression Methodologies}

In multiple regression, data from the independent variables can be entered in different orders; this characteristic implies the existence of different types of methodologies. Huck (2004) suggests that among all methodologies, the most commonly used are simultaneous, stepwise, and hierarchical. This study uses simultaneous and hierarchical methodologies.

Simultaneous Multiple Regression. In simultaneous multiple regression the data associated with the independent variables, in this study, leadership, curriculum, instruction, and demographic factors, are all entered at once, depending on the logic of a given research question. Huck (2004, p. 432) explains this technique with an analogy "to the 
process used in preparing vegetable soup where all ingredients are thrown into the pot simultaneously, stirred, and then cooked together." In this type of analysis, the multiple correlation coefficient $(R)$ measures the combined correlation of all independent variables to the dependent variable. Although $R$ is a significant indicator that sometimes appears in research papers, Huck states researchers are more prone to report $R^{2}$, which corresponds to the percentage of the variability in the criterion variable that can be accounted for by the predictor variables. In other words, $R^{2}$ represents the effect size for the independent variables. In this study, the resulting $R^{2}$ value represents the percentage of variability of the Academic Index scores attributed to those particular independent variables.

Hierarchical multiple regression. In hierarchical multiple regression, the data associated with the independent variables are entered in different stages, according to theoretical reasoning and the characteristics of the data. Huck (2004), with the soup analogy, suggests that hierarchical regression is designed to add the ingredients in different stages to favor certain flavors over the others or to avoid overcooking some components. He notes that the independent variables entered first are those corresponding with things the researcher wants to control. After explaining their portion of the variability in the dependent variable, the other variables are entered to see if they can contribute beyond the independent variables that went in first. Hierarchical regression generates several $R^{2}$ values, one for each stage of the analysis as independent variables are added. Adjusted $R^{2}$ values generate a form of percentage, indicating the degree to which variability in the dependent variable is explained by the set of independent variables included in the analysis (McKinney, 2007).

\section{Empirical Research Questions}

The four questions that guided this study are listed here again for the convenience 
of the reader. They depict the relationships among the variables (see Figure 1):

1. To what degree do demographic factors affect Standard 7 (Leadership), Standard 1 (Curriculum), Standard 3 (Instruction), and the Academic Index?

2. To what degree does Standard 7 (Leadership) affect Standard 1 (Curriculum), Standard (Instruction), and the Academic Index?

3. To what degree do Standard 1(Curriculum) and Standard 3 (Instruction) affect the Academic Index?

4. To what degree do Standard 1 (Curriculum) and Standard 3 (Instruction) mediate the effect of Standard 7 (Leadership) on the Academic Index, controlling for demographics?

Table 1 lists the multiple regressions to be calculated, by research question.

For the model investigated in this study (see Figure 1), there are two standards under the general heading, Mediating Factors. Although Curriculum and Instruction are clearly related, they are typically treated separately in schools. Efforts by educators to align the content defined by the curriculum and that actually covered through instruction reflect the importance of their continuity for accountability and the reality that they are distinct entities with respect to how teachers approach them and therefore need to be aligned (KDE, 2003). For this study, the two standards are examined separately. For RQ1 and $\mathrm{RQ} 3$, there are two univariate regressions rather than one multivariate analysis (see Table $1)$. 
Table 1

Variables and Type of Multiple Regression by Research Question

\begin{tabular}{|c|c|c|c|c|c|}
\hline \multirow{3}{*}{$\begin{array}{l}\text { Empirical } \\
\text { Research } \\
\text { Questions }\end{array}$} & \multicolumn{3}{|c|}{ Ind Variables } & \multirow{3}{*}{$\begin{array}{l}\text { Dependent } \\
\text { Variable(s) }\end{array}$} & \multirow{3}{*}{$\begin{array}{l}\text { Type of } \\
\text { Regression }\end{array}$} \\
\hline & Control & & Mediating & & \\
\hline & Variables & Leadership & Factors & & \\
\hline 1 & DEMV & & & LEAD & Simultaneous \\
\hline 1 & DEMV & & & CURR; INST & Simultaneous \\
\hline 1 & DEMV & & & AI & Simultaneous \\
\hline 2 & & LEAD & & CURR; INST & Simultaneous \\
\hline 2 & & LEAD & & $\mathrm{AI}$ & Simultaneous \\
\hline 3 & & & CURR; INST & AI & Simultaneous \\
\hline 4 & DEMV & LEAD & CURR; INST & AI & Hierarchical \\
\hline
\end{tabular}

Reliability and Validity of the Study

Reliability can be defined as "the consistency or stability of a measure or test from one use to the next. When repeated measurements of the same thing give identical or very similar results, the measurement instrument is said to be reliable"(Vogt, 1999, p. 245). Validity is "a term to describe a measurement instrument or test that accurately measures what it is supposed to measure; the extent to which a measure is free of systematic error" (Vogt, 1999, p. 301). For the purposes of this study, the concerns for reliability and validity of variables relates to construct validity, often examined empirically through psychometric analyses such as factor analysis (see Castello \& Osborne, 2005). Overall validity of a study is related to design issues (see Cook \& Campbell, 1979; MacCoun, 
Shadish, Cook, \& Campbell, 2003) and as such falls under the auspices of Kentucky's accountability model which represent a well-established design for school improvement.

The value of scientific research is partially dependent on the ability of individual researchers to demonstrate the credibility of their findings (LeCompte \& Goetz, 1982, p. 31). The Accountability Index (this study's dependent variable) has been under close scrutiny in Kentucky since the passage of KERA in 1990. The first accountability system, the Kentucky Instructional Results Information System (KIRIS) was replaced with the Commonwealth Accountability Testing System (CATS) because of questions regarding reliability and validity. L. S. Ennis (2002) states, however, that despite the criticisms about validity and reliability, "considerable efforts have been made to improve psychometric properties of the KIRIS test (later CATS)" (p. 195). The KDE (2006) states that there is evidence that the current CATS provides both valid and reliable data, based on extensive independent validity studies, a panel of the nation's top testing experts, and other national tests results. Specifically, the KDE has requested an independent firm, Human Resources Research Organization (HumRRO), to conduct extensive studies on CATS validity (B.C. Ennis, 2007). In addition, the KDE is in continuing contact with the National Technical Advisory Panel for Assessment and Accountability (NTAPAA), a panel of national testing experts that advises the Kentucky Legislature and Kentucky Board of Education on topics concerning technical aspects of CATS (KDE, 2006). In a KDE (2005b) report, the NTAPAA panel expressed "its support for the substantive work and engagement of stakeholders reflected throughout the process" (p. 1).

The scrutiny of validity and reliability for the CATS assessments has not carried through to the Scholastic Audit. Few studies have been conducted on the reliability and validity of the audit process. However, Koger and Thacker (2004) studied the relationship 
between the Academic Index and the Standards and Indicators for School Improvement document using data from the Scholastic Audit process and found that the teams involved in the audit process had been well trained in conducting audits and reviews. Koger and Thacker said "while there were no reliability measures taken to assess inter-rater reliability, all teams did use a standardized instrument and received training on conducting the audits/reviews" (p. i).

KDE conducted minimal analyses of the Scholastic Audit data, limited to establishing discrepancy scores (designated as Leverage or Variance Points) for each of the 88 indicators. Leverage points were those indicators for which there was a large discrepancy on indicator ratings (on the 4-point scale) between high performing and lower scoring schools on the Academic Index. Beyond the discrepancy studies and the additional work by Koger and Thacker (2004), no other validity/reliability investigations have been conducted by KDE or associates.

However, the set of completed dissertations (B.C. Ennis, 2007; McKinney, 2007; Saravia, 2008) and others in progress as directed by Drs. Miller and Smith at Western Kentucky University collectively provide strong evidence for both the psychometric quality and the external criterion validity of the nine SISI standards. At the elementary level, each of the nine standards has been subjected to factor analysis (McKinney, Miller, \& Smith, 2007; Smith, B. C. Ennis, Saravia, Miller, \& Wagner, 2008; Clayton, Harvey, Hammock, \& Miller, 2009); these results confirm that the set of indicators in each standard do function as a unitary construct as intended and designed by KDE. Multiple regressions utilizing these same factors similarly confirm the efficacy of these standards on the Academic Index, even after accounting for the influence of demographics. To date, no work has been completed at either middle or high school level. 
Although no validation exists on the Scholastic Audit at the high school level, there is closely related work. The current study replicates the dissertation of McKinney (2007). In the McKinney study, factor analyses were conducted on the three sets of indicators for three of the nine standards of the SISI document: Leadership (Standard 7), Curriculum (Standard 1), and Instruction (Standard 3). After the factor analysis (FA), Cronbach's alpha was conducted to examine the internal reliability of the factors. The FA resulted in a single factor emerging for each standard, explaining $54.6 \%, 56.9 \%$, and $51.0 \%$, respectively, of the variance among the items. Cronbach's coefficient alpha computed on these single factors yielded an overall composite value of $.915, .872$, and .857 , respectively, for the Leadership, Curriculum, and Instruction standards. The results reflected a high degree of internal reliability and confirmed the FA (McKinney, 2007, pp. 135-136).

Multiple regression analyses on the sets of variables of the study determined that demographic factors (School Size, Percent White, Appalachian, Percent Free and Reduced Lunch, County/ndependent district, Percent Gifted, Percent Disabled, and Year of Audit) had a small influence on the three standards examined in the study: Leadership (Standard 7), Curriculum (Standard 1), and Instruction (Standard 3). Effect sizes ranged from .08 to .16. Leadership had a modest influence on both Curriculum and Instruction (effect sizes of .35 and .36 , respectively) and a smaller direct impact on the Academic Index (.26). The two Academic Performance standards accounted for $36 \%$ of the variance on the Academic Index but only Instruction was statistically significant. The final hierarchical regression produced explained $71 \%$ variance on the Academic Index--the influence of Leadership on the Academic Index, controlling for demographic factors and as mediated by Curriculum and Instruction (McKinney, 2007, p. 136).

The overall results of the McKinney (2007) study confirmed the influence of the 
central research question, i.e., controlling for demographic factors, what is the effect on accountability outcomes of Leadership as mediated through Curriculum and Instruction? With the effect size of .71, the findings emphasize the role of Leadership on school improvement efforts at the elementary level through Instruction but not Curriculum. The results also confirmed the work of Murphy (2004), that a mediated effects model best describes the mostly indirect influence of the principal on achievement outcomes (McKinney, p. 137).

This parallel work at the elementary level provides assurance that the leadership, curriculum, and instruction standards have strong validity and reliability and can be utilized for the purposes of both research and school improvement efforts. The current study will provide further evidence on these same standards, at the high school level.

Ethical Issues

A basic premise of ethical research is that all respondents should be informed of the study's purpose, value, and confidentially. This study was presented to the Institutional Review Boards at University of Louisville and Western Kentucky University to assure that confidentiality and other human subject protection criteria were met, and that the study poses no more than minimal risk to any persons. Documentation of these presentations was included in the appendix section.

The database used in this study was compiled by the Kentucky Department of Education audit teams as Scholastic Audits were conducted. Thus, it is secondary data, meaning the researcher did not directly collect the information. There are no students, schools, or school districts identified; therefore, the research design poses no threat or risk. The project is consistent with the "Exempt" classification for research.

Summary 
This research is a quantitative analysis of secondary data collected by Scholastic Audit teams in the years 2000-2005 under the direction of the Kentucky Department of Education. The study examines the relationships between leadership, curriculum, instruction, and student achievement at the high school level with statistical emphasis on specific demographic variables.

In Kentucky, student achievement is measured by the Commonwealth Accountability Testing System (CATS). The overall Accountability Index includes results from the Kentucky Core Content Tests (KCCT), criterion-referenced assessments aligned with Kentucky standards; the CTBS 5 Survey Edition, a national norm-referenced test; and, non-academic factors. Tests administered annually to all Kentucky students constitute $90.25 \%$ of the Accountability Index. This portion of the Accountability Index-- the Academic Index--is based on the subject matter in the KCCT.

From annual test results and non-academic factors for each Kentucky school, a derived Academic Index score is determined. The Academic Index can dispose a school to a mandated (if not doing as well as expected) or voluntary Scholastic Audit. The stated purpose of the Scholastic Audits is "to provide a comprehensive review of a school's learning environment, organizational efficiency, and student academic performance" (KDE, 2004d, p. 1). The results of the audits are used to determine the support that schools need to improve student academic performance. Kentucky's Standards and Indicators for School Improvement (SISI) document was the assessment instrument used for the Scholastic AuditsReviews. The SISI document consists of nine standards subdivided into three groups: Academic Performance (Curriculum; Classroom Evaluation/Assessment; and Instruction), Learning Environment (School Culture, Student, Family and Community Support; and Professional Growth, Development, and Evaluation), and Efficiency 
(Leadership; Organizational Structure and Resources; and Comprehensive and Effective Planning) (KDE, 2004a).

For this study, the dependent variable is the Academic Index of consequence for each school and district, and is derived from the annual CATS assessment. This research attempts to determine the individual effect of each independent variable on the dependent variable to characterize the influences on the Academic Index (school achievement thus student achievement) of leadership as mediated by curriculum and instruction. Multiple regression was used to report the effect for demographic factors and establish any relationship for leadership with instruction and curriculum as well as on accountability. The direct effects of curriculum and instruction on the Academic Index are also investigated. There is an analysis of demographic variables on the Academic Index scores. The research uses simultaneous and hierarchical multiple regressions as the primary methods of computation. Descriptive statistics are reported along with psychometric analyses of the audit data for Standards 1,3, and 7. Additionally, validity and reliability of the secondary database are discussed. Lastly, ethical issues are addressed, including the evaluation by the Institutional Review Boards at the University of Louisville and Western Kentucky University. This study is the first to examine Kentucky's Scholastic Audit database at the high school. Previous work (see McKinney, 2007) examined these same variables and relationships for elementary level Scholastic Audits. 


\section{CHAPTER IV}

\section{RESULTS}

Introduction

The purpose of this study was: (a) to examine the effects of school level leadership on curriculum and instruction as they pertain to student success depicted by the Academic Index scores in Kentucky high schools, and (b) to investigate relationships, if any, that may exist among certain demographic factors such as school size, school setting, gender, and student achievement. Additionally, the intent is to add to the scholarship concerning the direct and indirect mediated actions of principals in Kentucky's high schools (as measured by Kentucky Scholastic Audits) as they function within a value-added high-stakes accountability milieu and affect student learning (measured by Academic Index scores performance assessment). In particular, the central research question established the parameters for this study: Based on Scholastic Audits, what are the effects of instructional leadership on Kentucky accountability scores, as mediated by curriculum and instruction?

To investigate the empirical research questions (specific relationships designed to answer the central research question), the researcher used secondary data from the Kentucky Department of Education (KDE) compiled through the Scholastic Audit process and supplemented by information from the Kentucky Performance Report (KPR) database. The Scholastic Audits are conducted by KDE trained audit/review teams that use the Standards and Indicators for School Improvement (SISI) document (KDE, 2003) to evaluate progress toward reaching the statewide goal for each Kentucky school of an 
Accountability score of 100 by the year 2014. Once collected, the Scholastic Audit data were electronically compiled by $\mathrm{KDE}$ and made available to the public for viewing or purposes of research. This controlled system minimized or eliminated many problems associated with data collection. In this study, the researcher found no missing data or unexpected values. The Kentucky Performance Report contains demographic and academic data that are available for each school in the state. Specifically, the data used for this study were derived from audits conducted in 83 of the 224 Kentucky high schools from 2001 to 2005 .

The relationships depicted in Figure 1 illustrate the different types of variables and their assumed linkages (assumptions based on previous work of B. C. Ennis, 2007; McKinney, 2007; and Saravia, 2008). Demographic characteristics are used as control variables. Standard 7 (Leadership) is the independent variable while Standard 1 (Curriculum) and Standard 3 (Instruction) serve to mediate the effects of Leadership (cf. Murphy, 2004). The Academic Index, as determined by the Commonwealth Accountability Testing System (CATS), is the school level dependent variable.

After calculating descriptive statistics, exploratory factor analysis was conducted to determine whether the indicators representing Standard 1 (Curriculum) would load into a single factor that could be used to create a factor-weighted, summated scale measuring Standard 1 (Curriculum). Likewise, exploratory factor analyses were conducted to determine if the indicators of Standard 3 (Instruction) and Standard 7 (Leadership) could be considered a single factor. In addition, Cronbach's alpha was computed to determine the internal reliability of the factors produced by the factor analysis. Also, a correlation matrix of the dependent, independent, and control variables was created. Although this matrix is a precursor to the regression analysis used to test the research questions, the inter- 
correlations provide further evidence of the construct validity of these measures.

To test the research questions, multiple regression analysis was used to determine the extent to which each independent variable affects the particular dependent variable for a given equation, controlling for the other independent variables in the model.

Simultaneous multiple regression analysis was used to determine the relationships outlined in Research Questions 1-3. A three-step hierarchical multiple regression was used to explore the full model as stated in Research Question 4. The remaining sections of this chapter--Descriptive Statistics, Psychometric Analyses, Research Questions, and Summary--detail the results of these analyses.

\section{Descriptive Statistics}

Descriptive statistics are reported for the demographic data, Standard 7 (Leadership), Standard 1 (Curriculum), Standard 3 (Instruction), and school achievement scores (Academic Index). Summaries for each variable are reported. This study included the 83 Kentucky high schools audited during 2001-2005. Scores from the Kentucky Core Content Test (KCCT) for grades 10 and 11 produced each school's Academic Index. The demographic variables reflect these two grades.

The Kentucky high schools were audited in five rounds from 2001 to 2005. The 2001 round provided 18 cases; 2002, 12 cases; 2003, 24 cases; 2004, 11 cases; and 2005, 18 cases $(N=83)$. The sample included all audited schools containing grades $9-12$ during the study period. Student populations were confined to grades 10 and 11 since only these student scores generate the Academic Index for each school. The KDE performed a total of 376 scholastic audits and reviews during the five rounds from 2001 to 2005 , including elementary, middle, and high schools; this number also includes two P-12 schools.

\section{Dependent Variable}


Descriptive statistics for the Academic Index, the dependent variable, are presented in this section. The Academic Index constitutes $90.25 \%$ of the overall Accountability Index. The Academic Index is a composite score across the individual students in a school for all content areas assessed by the CATS, and weighted and combined into a single figure. The goal for each school in Kentucky is to reach proficiency, a score of 100 out of a possible 140 , by the year 2014 . The lowest performing school in the study had an overall Academic Index of 36.8. The highest performing school in the study had an overall Academic Index of 76. The sample contained both low-performing and more successful schools. The Academic Index mean and standard deviation for the sample $(N=83)$ are 59 and 9.84 , respectively. The mean value reflects reality that most of the schools in the study are struggling schools because all Level 3 schools (the lowest scoring in Kentucky) were mandated to complete the audit as part of the state's school improvement effort. In addition, the standard deviation of 9.84 suggests there is substantial variation between the scores of these schools.

\section{Independent Variables}

For this study, the Independent Variables are divided into three distinct groupings: Control, Leadership, and Mediating Factors. These three headings are consistent with social science research on distinctions among independent variables. Bloom (1980) indicates that control variables are non-alterable givens which the researcher cannot manipulate, typically some combination of socio-demographic or personal markers. Their prior effect on school and life outcomes is both considerable and important to understanding the net influences of other predictors. Alterable variables represent factors that professionals or researchers can change or that vary across circumstances. This is typically the target of the research, in this instance, the effects of Leadership in Murphy's 
(2004) model. The third subgrouping is the Mediating Factors, an indirect linkage between the target variable(s) and the outcomes measures.

In the current study, these three types of independent variables are placed in the context of the Standards and Indicators for School Improvement. The KDE classified the nine standards of the SISI document into three heading--Academic Performance (Standards 1, 2, \& 3), Learning Environment (Standards 4, 5, \& 6), and Efficiency (Standards 7, 8, \& 9). This study examines Standard 7 (Leadership), the target construct, along with Standard 1 (Curriculum) and Standard 3 (Instruction)--both hypothesized as Mediating Factors in Murphy's (2004) mediated effects model of instructional leadership. Only the descriptive statistics for the demographic controls and the dependent variable are presented here. The descriptives for Standard 7 (Leadership), Standard 1 (Curriculum), and Instruction (Standard 3) are presented in the section below, Psychometric Analysis. Control

The control variables for this study include: Appalachian (APP), County/ Independent district (CTY), Percent Disability (\%DIS), Percent Free/Reduced Lunch (\%FRL), Percent Gifted (\%GFT), Percent Limited English Proficiency (\%LEP), School Size (SIZE), Percent White (\%WH), and Year of Audit (YR). These nine variables were selected because socio-demographic indicators commonly utilized in studies of school outcomes. In addition, they represent the larger set of demographic variables tested collectively in the three elementary level investigations of the Scholastic Audit database (B. C. Ennis, 2007; McKinney, 2007; Saravia, 2008). Highlights from Table 2, the descriptive statistics for these variables, are noted. School Size was measured by the enrollments in grades 10 and 11; the standard deviation reflects the substantial disparity in the size of the schools in the database for this study. The statistical mean for 
$\%$ Free/Reduced Lunch, at $41.55 \%$, is below the state average of approximately $50 \%(\mathrm{~S} . \mathrm{K}$. Miller, Smith, \& Ennis, 2006). The values indicate that less than $50 \%$ of the audited schools are in the Appalachian Area (APP) and more than three-fourths are County as opposed to Independent school districts.

Table 2

Descriptive Statistics for Demographic Controls $(N=83)$

\begin{tabular}{lrcccc} 
Measure & \multicolumn{1}{c}{ M } & \multicolumn{1}{c}{$S D$} & Minimum & Maximum & Range \\
\hline APP & .41 & .49 & 0.00 & 1.00 & 1.00 \\
CTY & .83 & .38 & 0.00 & 1.00 & 1.00 \\
\%DIS & 11.53 & 7.27 & 1.37 & 49.54 & 48.17 \\
\%FRL & 41.55 & 16.88 & 11.83 & 77.86 & 66.02 \\
\%GIF & 12.52 & 8.21 & .00 & 31.25 & 31.25 \\
\%LEP & 11.53 & 7.27 & 1.37 & 49.54 & 48.17 \\
SIZE & 334.11 & 163.85 & 36.00 & 802.00 & 766.00 \\
\%WH & 87.41 & 17.54 & 11.50 & 100.00 & 88.50 \\
YR & 2001.99 & 1.43 & 2000. & 2004. & 4.00 \\
\hline
\end{tabular}

Psychometric Analysis

Eighty-eight indicators were established within the nine standards of the SISI by $\mathrm{KDE}$. These indicators represent behaviors or attitudes that measure the success a school is achieving in the respective standard. Although the indicators were selected based on theoretical and empirical evidence, $\mathrm{KDE}$ has not conducted formal psychometric 
assessment of the indicators to determine if any underlying structure can be detected among the indicators. Three types of computations were included as part of the overall set of procedures: factor analysis, internal scale reliability, and inter-scale correlations.

\section{Factor Analysis}

Factor analysis (FA) is a procedure used to determine the extent to which shared variance, or measurement overlap (Williams, 1992), exists among a set of items. The calculations arrange them into subsets of separate and distinct constructs or factors (Nunnally \& Bernstein, 1994). More specifically, factor analysis is often used in the development of indexes and scales and constitutes a form of data reduction. However, at times FA can be used to mine a common factor (or factors) from the larger set of items that may or may not represent scales. Either way, the intention is to produce a more frugal set of constructs to represent the larger set (Pett, Lackey, \& Sullivan, 2003).

Many exploratory factor analysis researchers utilize a procedure called principal components analysis. The principal component analysis method evaluates all sources of variability for each variable, with the resulting factors representing the underlying components (Lynes, 2008). If there are multiple factors or components identified, the initial factor loadings are subjected to a procedure called rotation. The goal of the rotation is to clarify the data structure. Costello and Osborne (2005) suggest that it is best to perform an oblique rotation, when rotation is necessary. Rotation cannot improve the basic aspects of the analysis such as the amount of variance but oblique rotation does allow the factors to correlate, as compared with orthogonal rotation, which works under the assumption that underlying factors are not correlated (Costello \& Osborne). The oblique option is commonly utilized in the social sciences where constructs are typically related (Pett et al., 2003). The principal component analysis is the default setting for SPSS, but 
oblique rotation is not. Subsequently, these procedures are used in this study. Analyzing the SISI Document

Separate exploratory factor analyses were performed on the items comprising Standards 1,3 , and 7 . In the current research model, each standard was analyzed separately. The primary consideration has to do with the relationship between factor analysis and theory. There are two types of factor analysis: exploratory and confirmatory. Confirmatory factor analysis is generally used in areas where there is a well-established theoretical understanding of the underlying latent factors hypothesized for the variables and typically requires many cases, several statistical assumptions, and more complex computations involving covariance structures (Pett et al., 2003). This type of factor analysis is beyond the scope of the current study in part due to the small sample size. Beyond the limited $N$, the SISI document simply does not meet the theoretical rigor suggested for confirmatory factor analysis.

The second type of factor analysis is exploratory. Exploratory factor analysis is generally used when there are suspected, but poorly understood relationships among several indicators. This situation is common when there is limited empirical work on a set of indicators. Exploratory factor analysis may be considered as a somewhat more quasitheoretical check on items created to function as scales, based loosely on a priori considerations, in this case the work by KDE detailing the various literatures upon which the restrictive standards were based (KDE, 2003). Although this second approach falls short of the theoretical rigor of confirmatory factor analysis, it is clearly within accepted statistical procedures for exploring a set of constructs. This approach, checking to see if scales hang together as intended, is appropriate where instruments are in early stages of development and there has been little or no previous psychometric analyses performed to 
investigate their efficacy regarding the constructs intended (cf. Nunnally \& Bernstein, 1994).

Exploratory factor analysis is the appropriate approach to examining the standards created by KDE researchers. The original SISI document (KDE, 2003) details the separate literatures that supported each of the nine distinct but related standards, drawn piecemeal from educational and organizational theory. Each standard was also hypothesized to represent an underlying structure believed to be important for school improvement. Given an underlying, but untested, quasi-theoretical basis for each separate standard, combining the indicators of the three standards together for analysis would disregard the original development of the nine standards and their respective indicators.

It is also relevant that the purpose of the current study is not a rigorous examination of the underlying factor structure of the SISI document. Rather, the procedures examining validity and reliability of the three standards utilized in this study examine the psychometric properties of the variables that are incorporated into a test of Murphy's mediated effects model of instructional leadership. That purpose dictates a more pragmatic approach to the validity of the standards in question than would the intent to provide a rigorous test of the SISI document as an end in itself.

Of less importance is sample size. The current small sample also works against analyzing the three standards together. Costello and Osborne (2005) find that the majority of factor analyses have a ratio of approximately 10 cases for every item or indicator included for analysis. This would mean that eight or fewer indicators would be optimal for factor analysis with this sample. With 26 indicators across the three standards, an overall FA would fall far short of this optimal ratio.

\section{Procedures Used}


Using factor scores derived from the factor analyses of Standards 1, 3, and 7, factor weighted scales were created for each standard. The default in most statistical software packages is to retain all factors with eigenvalues greater than 1.0. However, there is broad consensus in the literature that this is among the least accurate method so alternative tests for factor retention (scree tests, Velicer's MAP criteria, and parallel analysis) are recommended (Velicer \& Jackson, 1990). The Velicer's MAP and parallel analysis are typically not available in most frequently used statistical software. Therefore, the best choice for researchers is the scree test, a graph of the eigenvalues (Costello \& Osborne, 2005). The scree test, available in the SPSS statistical software, was the alternative test for factor retention used in this study.

Finally, Cronbach's (1951) coefficient alpha was calculated to examine the internal reliability from the factor analyses. Cronbach's alpha, a numerical coefficient of reliability (Santos, 1999), measures the internal consistency of a set of items. When the items have been selected based on theoretical rationale, the underlying factor for the scale is deduced. Although a reliability procedure, a high coefficient alpha indicates that the items are measuring the same construct. A rule of thumb is that a coefficient of .7 or higher is generally an indication of internal consistency although reliabilities as low as .6 may be acceptable for exploratory research (Nunnally \& Bernstein, 1994). Along with the factor analysis results for these scales, this procedure can also be interpreted as supportive evidence for construct validity.

A correlation matrix, based on the composite scale values for the constructs derived from factor analysis and the operational definitions for the demographic factors and the dependent variable, can be constructed from the variables in a study to provide further evidence of external criterion validity (Nitko, 2001). Correlations measure the strength and 
direction of association between pairs of variables. If two variables have no relationship then they would have a .00 correlation. Conversely, if they had a perfect relationship, they would have a correlation of 1.00 or -1.00 depending on the direction of the relation. Interscale correlations were then examined to assess the degree of independence among the three standards. Ideally, scales conceptualized as distinct but related should produce correlations in the .3 to .75 range (Nunnally \& Bernstein, 1994; Pett et al., 2003).

Correlations less than that indicate independence among the scales but raise questions as to their interdependence. Stronger correlations raise concerns as to whether the scales are distinct as opposed to representing a single larger construct. The full correlation matrix also constitutes the data base from which subsequent multiple regressions are calculated.

\section{Leadership}

In this study, the only standard included from the Efficiency grouping (Standards 7 , 8, and 9 from the SISI document) is Standard 7 (Leadership). As discussed in Chapter III, the audit scores for each standard that had been assessed on the 1-4 scale were converted to scores of $0,2,5$, and 7 to mirror the larger incremental movement from 2 to 3 compared to the other score transitions. McKinney (2007) details the rationale for this transformation and cites KDE personnel to support this. That rationale applies equally to this study and is quoted at length here.

After considerable discussion with persons knowledgeable of Kentucky's accountability and audit procedures (Bill Insko, Lou Spencer, and Robert Wetter, personal communication, August 5, 2005), the decision was made that the original audit scores of 1-4 would be converted to scores of $0,2,5$, and 7 . These values seem to represent more accurately the protracted difficulty of advancing from limited development (2) to fully functioning (3). This seems to parallel the larger gap in Kentucky's scoring rubric associated with moving students from Apprentice to Proficient on the four criterion levels of achievement for the 140 point scale for the KCCT (Novice, 0-39; Apprentice, 40-99; Proficient, 100-139; and Distinguished, 140). (p. 81) 
Exploratory factor analysis was then used to determine what, if any, underlying structures exist for the 11 indicators of Standard 7 that are hypothesized to represent leadership. Principal components analysis was the extraction method used, with oblique rotation. As stated previously, there are several different practices used by researchers to determine the appropriate number of components to represent the underlying factors produced in these computations. Costello and Osborne (2005) argue that using the bend in the scree plot is the best practice available when using standard statistical analysis software like SPSS. However, the default in SPSS is that the appropriate solution is determined by accepting any factor with an eigenvalue above one. These alternative guidelines lead to potentially different underlying structures. In such cases, Costello and Osborne suggest that both solutions be examined and the cleaner solution chosen, where a "clean" factor structure is indicated by high component loadings (above .30), no cross-loadings, and no components with three or fewer items. Because alternative solutions may represent some combination of these criteria, the analyst is sometimes forced to weigh trade-offs. The reasoning for these steps should be laid out for the reader so that the decision process is transparent.

The original principal components analysis for the 11 indicators of Standard 7 produced a two component solution (see Appendix H, Table H1). Both the Factor Structure Matrix and the Factor Pattern Matrix are included; primary interpretation comes from the Factor Pattern Matrix values. The solution demonstrates five indicators clearly load on component one, five load on component two, and one cross-loads on both components. In contrast, Table 4 presents the factor loadings for a solution forced to one component, with values ranging from .777 to .515 . Given the criteria from Costello and Osborne (2005), the single factor solution (Table 4) is preferable to the two components, with a cross-loading 
item in Table H1. Accordingly, the single component solution was selected to represent the underlying structure among the indicators for Standard 7.

The eigenvalues for the principal components analysis are presented in Table 3 , with $45.44 \%$ of the variance explained for the single factor solution.

Table 3

Total Variance Explained for Standard 7, Leadership

\begin{tabular}{|c|c|c|c|c|c|c|}
\hline \multirow{3}{*}{ Component } & \multicolumn{3}{|c|}{ Initial Eigenvalues } & \multicolumn{3}{|c|}{ Extraction Sums of Squared Loadings } \\
\hline & Total & $\%$ of & $\overline{\text { Cumulative }}$ & Total & $\%$ of & Cumulative \\
\hline & & Variance & $\%$ & & Variance & $\%$ \\
\hline 1 & 4.998 & 45.439 & 45.439 & 4.998 & 45.439 & 45.439 \\
\hline 2 & 1.286 & 11.694 & 57.133 & & & \\
\hline 3 & .892 & 8.111 & 65.244 & & & \\
\hline 4 & .759 & 6.903 & 72.147 & & & \\
\hline 5 & .629 & 5.721 & 77.868 & & & \\
\hline 6 & .580 & 5.272 & 83.140 & & & \\
\hline 7 & .518 & 4.709 & 87.849 & & & \\
\hline 8 & .431 & 3.920 & 91.769 & & & \\
\hline 9 & .359 & 3.267 & 95.036 & & & \\
\hline 10 & .285 & 2.587 & 97.623 & & & \\
\hline 11 & .261 & 2.377 & 100.000 & & & \\
\hline
\end{tabular}

Note. Extraction Method: Principal Component Analysis.

Figure 2, the scree plot of the eigenvalues for Standard 7 (Leadership), provides additional evidence on which solution is more appropriate as to the number of factors 
(Costello \& Osborne, 2005), which should be the same as the number of eigenvalues above the bend in the plot. These criteria indicated a single component solution was appropriate.

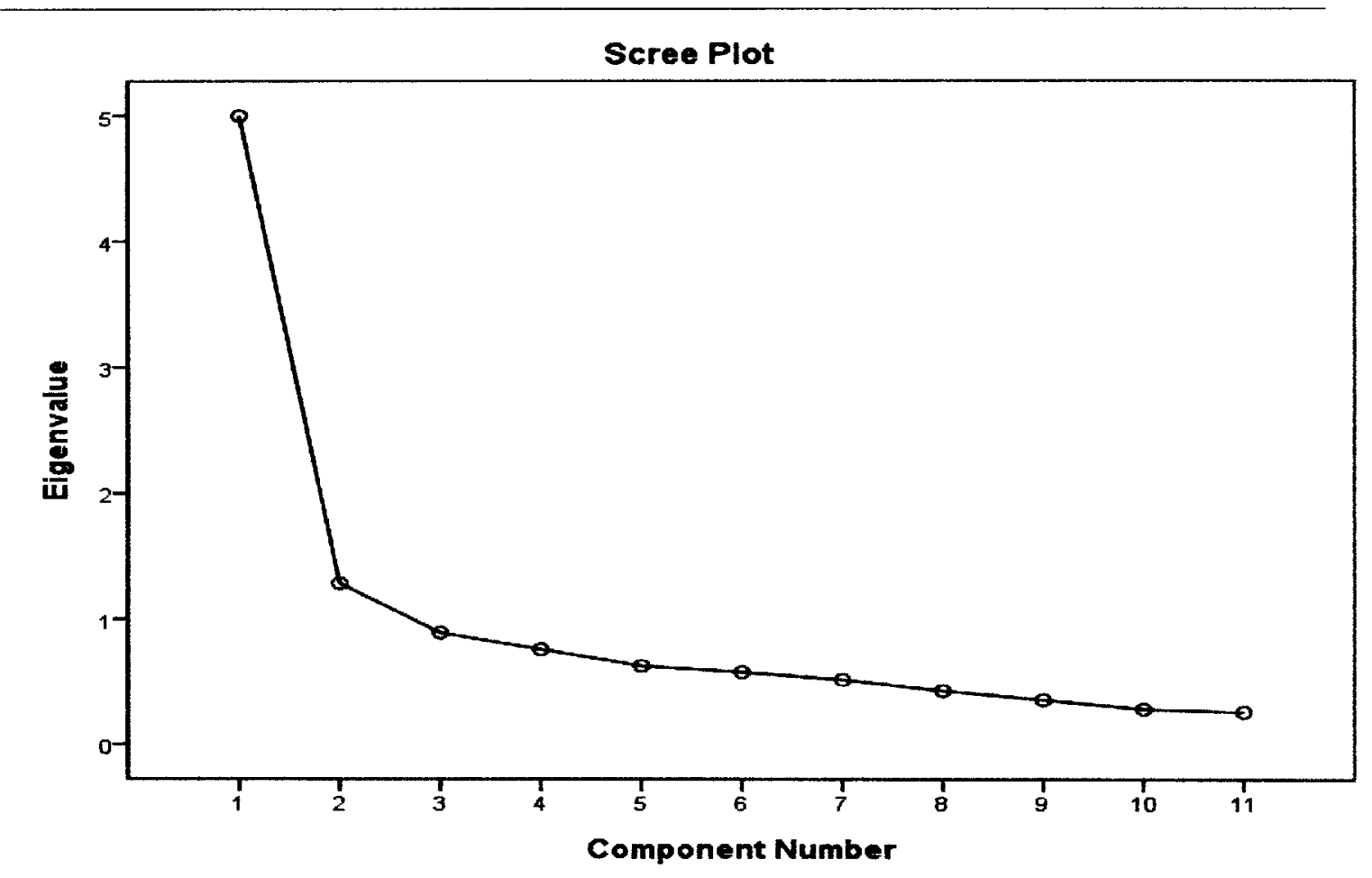

Figure 2. Scree plot for Standard 7, Leadership.

Table 4 lists the 11 indicators and respective factor loadings for the single component solution to Standard 7, Leadership. Factor loadings can be considered the correlation between each indicator and the underlying factor (Mertler \& Vanetta, 2005). As evidenced from the factor analysis, four of the 11 indicators cluster very tightly as they measure common entities within the Leadership component. These clustered indicators (7.1.g, 7.1.k, 7.1.b, and 7.1.e) range from .777 to .744 . The other indicators also contribute to the overall factor but are not as closely correlated to the underlying Leadership factor as the first four. 
Table 4

Factor Loadings for Standard 7, Leadership

Indicators $^{\mathrm{a}}$

Loadings

7.1.g Leadership plans and allocates resources, monitors progress, provides

organizational infrastructure, and removes barriers in order to sustain

continuous school improvement.

7.1.k There is evidence that the principal demonstrates leadership skills in the areas of academic performance, learning environment, and efficiency.

7.1.b Leadership decisions are focused on student academic performance and are data-driven and collaborative.

7.1.e Leadership ensures all instructional staff has access to curriculum related materials and the training necessary to use curricular and data resources relating to the learning goals for Kentucky public schools.

7.1.d There is evidence that the school/district leadership team disaggregates data for use in meeting the needs of a diverse population, communicates the information to school staff and incorporates the data systematically into the school's plan (CSIP).

7.1.i Leadership provides a process for the development and the implementation of council policy based on anticipated needs.

7.1.f Leadership ensures that time is protected and allocated to focus on curricular and instructional time.

7.1.j There is evidence that the SBDM (Site Based Decision Making) council has an intentional focus on student academic performance.

7.1.a. Leadership has developed and sustained a shared vision.

7.1.h The school/district leadership provides the organizational policy and resource infrastructure necessary for the implementation and maintenance of a safe and effective learning environment.

7.1.c There is evidence that all administrators have a growth plan focused on the development of effective leadership skills.

${ }^{\mathrm{a}} \mathrm{KDE}(2004 \mathrm{~b}, \mathrm{pp} .32-33)$. 
Cronbach's coefficient alpha was then computed on the indicators to determine internal reliability of the suggested scale (see Table 5). Although it would be expected that items from a construct derived from or supported by exploratory would exhibit adequate internal reliability, that is an empirical matter to be tested. Cronbach's alpha values vary across the results of FA and occasionally a factor derived from exploratory factor analysis does not exhibit adequate internal reliability (cf. Lynes, 2008). For Standard 7, the composite alpha of .862 indicated a high degree of internal reliability. Along with the FA, this supports that the 11 indicators for the scale constitute strong, consistent measurement of the school leadership construct. The alpha with item deleted column $(\alpha-d)$ reveals that the internal reliability of the standard would change very little if any single item were removed. The item that fits least well is 7.1.c. $(\alpha-d=.874)$. Conversely, removing items 7.1.g. and 7.1.k. would lower the overall Cronbach's alpha the most $(\alpha-d=.853$ and .854 , respectively). This result matches the factor analysis as the two items most strongly correlated with the underlying mathematical construct were indeed 7.1.g. and 7.1.k. (from Table 4). The mean and standard deviation for both the individual items and the composite scale $(M=2.3, S D=1.53)$ also reveal good psychometric properties. 
Table 5

Internal Reliability and Item Characteristics for Standard 7, Leadership $(N=83)$

\begin{tabular}{lllll}
\hline Indicator & $M$ & $S D$ & Range & $\alpha-\mathrm{d}^{\mathrm{a}}$ \\
\hline 7.1.a & 1.58 & 1.40 & 5 & .866 \\
$7.1 . \mathrm{b}$ & 2.45 & 1.37 & 7 & .858 \\
7.1.c & 2.35 & 1.74 & 5 & .874 \\
7.1.d & 2.30 & 1.51 & 7 & .862 \\
7.1.e & 2.82 & 1.52 & 7 & .857 \\
$7.1 . \mathrm{f}$ & 1.94 & 1.58 & 5 & .863 \\
$7.1 . \mathrm{g}$ & 2.48 & 1.53 & 7 & .853 \\
$7.1 . \mathrm{h}$ & 3.25 & 1.82 & 7 & .871 \\
$7.1 . \mathrm{i}$ & 1.72 & 1.46 & 5 & .864 \\
$7.1 . \mathrm{j}$ & 1.70 & 1.47 & 5 & .864 \\
$7.1 . \mathrm{k}$ & 2.31 & 1.48 & 5 & .854 \\
Total & 2.26 & 1.53 & 5.90 & $.862^{\mathrm{b}}$ \\
\hline $\mathrm{a} \alpha-\mathrm{d}=$ alpha with & & & & \\
\hline
\end{tabular}

${ }^{b}$ Value for $\alpha-d$ for Total is Cronbach's coefficient alpha for the entire scale.

\section{Mediating Factors}

From the Academic Performance section of the nine standards of the SISI document, Standard 1 (Curriculum) and Standard 3 (Instruction) are included in this study as mediating variables in Murphy's (2004) model of indirect leadership effects. To determine if any underlying components exist for the seven indicators of Standard 1, 
Curriculum, principal components analysis was conducted using an oblique rotation. The original calculations produced a two component solution (see Appendix H, Table H2). Again, looking at the indicators thought to measure curriculum given this two component solution, three indicators clearly load on component one, two indicators load on component two, and two indicators cross-load, i.e., those particular component loadings are high enough to appear in both factors (Indicators 1.1a and 1.1f). Given the component structure presented in Table $\mathrm{H} 2$ which has cross-loading and few items in each component compared to the single component solution presented in Table 7 (forced into one component) with high loadings ranging from .818 to .440 , the single component solution was selected as the most appropriate representation of the underlying structure among the indicators.

The eigenvalues for the principal component analysis are listed in Table 6. Both components 1 and 2 have eigenvalues above one ( 3.701 and 1.013, respectively), but as described above in the comparison of the one and two component solutions (Table 7 and Table H2, respectively), the single component better meets the criteria of Costello and Osborne (2005). The computation of the eigenvalue for component one explained 52.9\% of the variance among this set of seven indicators for Standard 1, Curriculum. 
Table 6

Total Variance Explained for Standard 1, Curriculum

\begin{tabular}{|c|c|c|c|c|c|c|}
\hline \multirow{3}{*}{ Component } & \multicolumn{3}{|c|}{ Initial Eigenvalues } & \multicolumn{3}{|c|}{ Extraction Sums of Squared Loadings } \\
\hline & Total & $\%$ of & Cumulative & Total & $\%$ of & Cumulative \\
\hline & & Variance & $\%$ & & Variance & $\%$ \\
\hline 1 & 3.701 & 52.877 & 52.877 & 3.701 & 52.877 & 52.877 \\
\hline 2 & 1.013 & 14.465 & 67.342 & & & \\
\hline 3 & .773 & 11.044 & 78.385 & & & \\
\hline 4 & .509 & 7.273 & 85.658 & & & \\
\hline 5 & .448 & 6.398 & 92.056 & & & \\
\hline 6 & .342 & 4.887 & 96.943 & & & \\
\hline 7 & .214 & 3.057 & 100.000 & & & \\
\hline
\end{tabular}

Note. Extraction Method: Principal Component Analysis.

Figure 3 displays the scree plot of the eigenvalues for Standard 1, Curriculum. According to Costello and Osborne (2005), the scree plot is the best solution for choosing the number of factors. The factor(s) above the bend in the plot should match the number of factors determined by eigenvalue calculations. This criterion for Standard 1, Curriculum, indicated a single component solution was appropriate. 


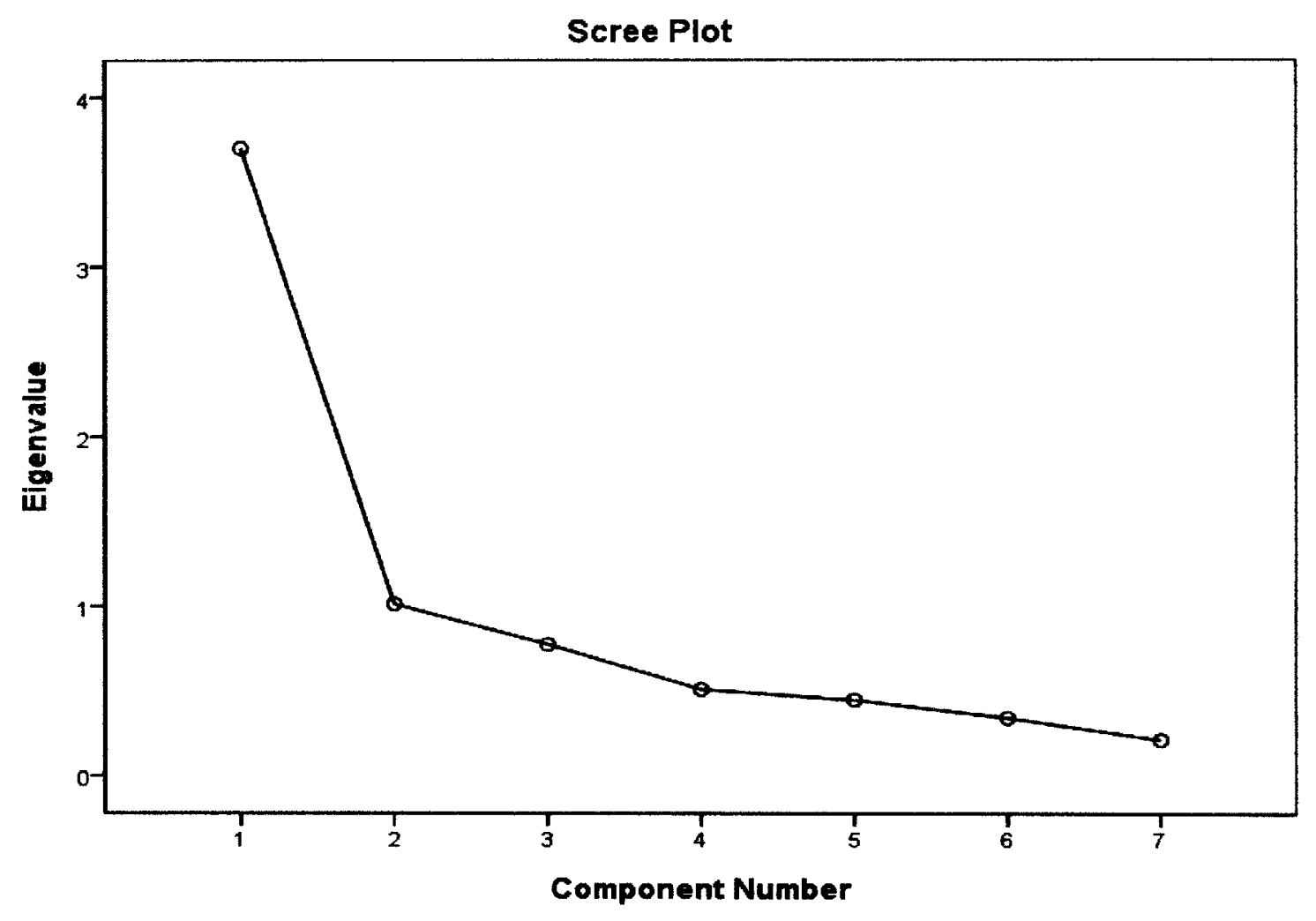

Figure 3. Scree plot for Standard 1, Curriculum.

Table 7 lists the seven indicators and factor loadings for Standard 1, Curriculum for the single component solution. Although 1.1.c has the high loading (.818), five of the seven correlate with an underlying factor at .729 or above (1.1a, 1.1.b, 1.1.f, 1.1.d. and 1.1.g). Indicator 1.1.g is not quite as connected (.618) but still is a strong contributor. The least tightly bound is 1.1 .e which has a long range focus compared with the greater schoolwide emphasis of the others. However, even this indicator loads well above suggested minimum loadings. 
Table 7

Factor Loadings for Standard 1, Curriculum

Indicators $^{\mathrm{a}}$

Loadings

1.1.c The district initiates and facilitates discussions between schools in the

district in order to eliminate unnecessary overlaps and close gaps.

1.1.a There is evidence that the curriculum is aligned with the Academic Expectations, Core Content for Assessment, Transformations and the Program of Studies.

1.1.b The district initiates and facilitates discussions among schools regarding curriculum standards to ensure they are clearly articulated across all levels ( $\mathrm{P}$ 12).

1.1.f There is in place a systematic process for monitoring, evaluating, and reviewing the curriculum.

1.1.d There is evidence of vertical communication with an intentional focus on key curriculum transition points within grade configuration (e.g., from primary to middle and middle to high).

1.1.g The curriculum provides access to a common academic core for all students.

1.1.e The school curriculum provides specific links to continuing education, life, and career options.

${ }^{a} \mathrm{KDE}$ ( 2004b, pp. 32-33).

Cronbach's alpha was calculated to assess the scale reliability. Table 8 presents the descriptive statistics and reliability analyses on the seven indicators for Standard 1 (Curriculum). Removing item 1.1.e would increase the scale reliability to .861 . However, the gain in reliability is not worth the loss of integrity to the scale as intended by KDE and confirmed by both factor analysis and scree plot. Further, the composite alpha of .824 reflects a very adequate degree of internal reliability. Both individual items and the composite scale indicate good psychometric properties; the composite scale has mean and 
standard deviation of 2.01 and 1.53 , respectively.

Table 8

Internal Reliability and Item Characteristics for Standard 1, Curriculum $(N=83)$

\begin{tabular}{lllll}
\hline Indicator & $M$ & $S D$ & Range & $\alpha-\mathrm{d}^{\mathrm{a}}$ \\
\hline 1.1.a & 2.30 & 1.67 & 5 & .808 \\
$1.1 . \mathrm{b}$ & 2.05 & 1.68 & 5 & .815 \\
$1.1 . \mathrm{c}$ & 2.04 & 1.63 & 5 & .808 \\
$1.1 . \mathrm{d}$ & 1.75 & 1.31 & 5 & .823 \\
$1.1 . \mathrm{e}$ & 2.60 & 1.39 & 7 & .861 \\
1.1.f & 1.60 & 1.51 & 5 & .815 \\
1.1.g & 1.75 & 1.31 & 5 & .840 \\
Total & 2.01 & 1.53 & 5.29 & $.824^{\mathrm{b}}$ \\
\hline a & & & &
\end{tabular}

$\overline{\mathrm{a}} \alpha-\mathrm{d}=$ alpha with item deleted.

${ }^{b}$ Value for $\alpha-d$ for Total is Cronbach's coefficient alpha for the entire scale.

Standard 3 (Instruction) of the SISI document is composed of eight indicators. A single factor was produced from the original factor analysis and the calculation of the eigenvalues (displayed in Table 9) explained $48.2 \%$ of the variance internal to the standard. Only one component had an eigenvalue greater than one (3.856), supporting the one factor solution. 
Table 9

Total Variance Explained for Standard 3, Instruction

\begin{tabular}{crrrrrr}
\hline & \multicolumn{3}{c}{ Initial Eigenvalues } & \multicolumn{3}{c}{ Extraction Sums of Squared Loadings } \\
\cline { 2 - 6 } Component & Total & $\begin{array}{c}\text { \% of } \\
\text { Variance }\end{array}$ & $\begin{array}{c}\text { Cumulative } \\
\%\end{array}$ & Total & $\begin{array}{c}\text { \% of } \\
\text { Variance }\end{array}$ & $\begin{array}{c}\text { Cumulative } \\
\%\end{array}$ \\
\hline 1 & 3.856 & 48.201 & 48.201 & 3.856 & 48.201 & 48.201 \\
2 & .940 & 11.749 & 59.949 & & \\
3 & .821 & 10.266 & 70.215 & & \\
4 & .692 & 8.650 & 78.865 & & \\
5 & .526 & 6.577 & 85.443 & & \\
6 & .437 & 5.468 & 90.910 & & \\
7 & .413 & 5.169 & 96.079 & & \\
8 & .314 & 3.921 & 100.000 & \\
\hline
\end{tabular}

Note. Extraction Method: Principal Component Analysis.

The scree plot for Standard 3, Instruction, a visual depiction of the eignevalues, is displayed in Figure 4. Researchers use this graphical representation to determine the natural bend or break point in the data where the curve flattens out. The number of data points above the break is usually the number of factors to retain (Costello \& Osborne, 2005). The scree plot suggests that a single factor solution is appropriate, consistent with only one eigenvalue above one. 


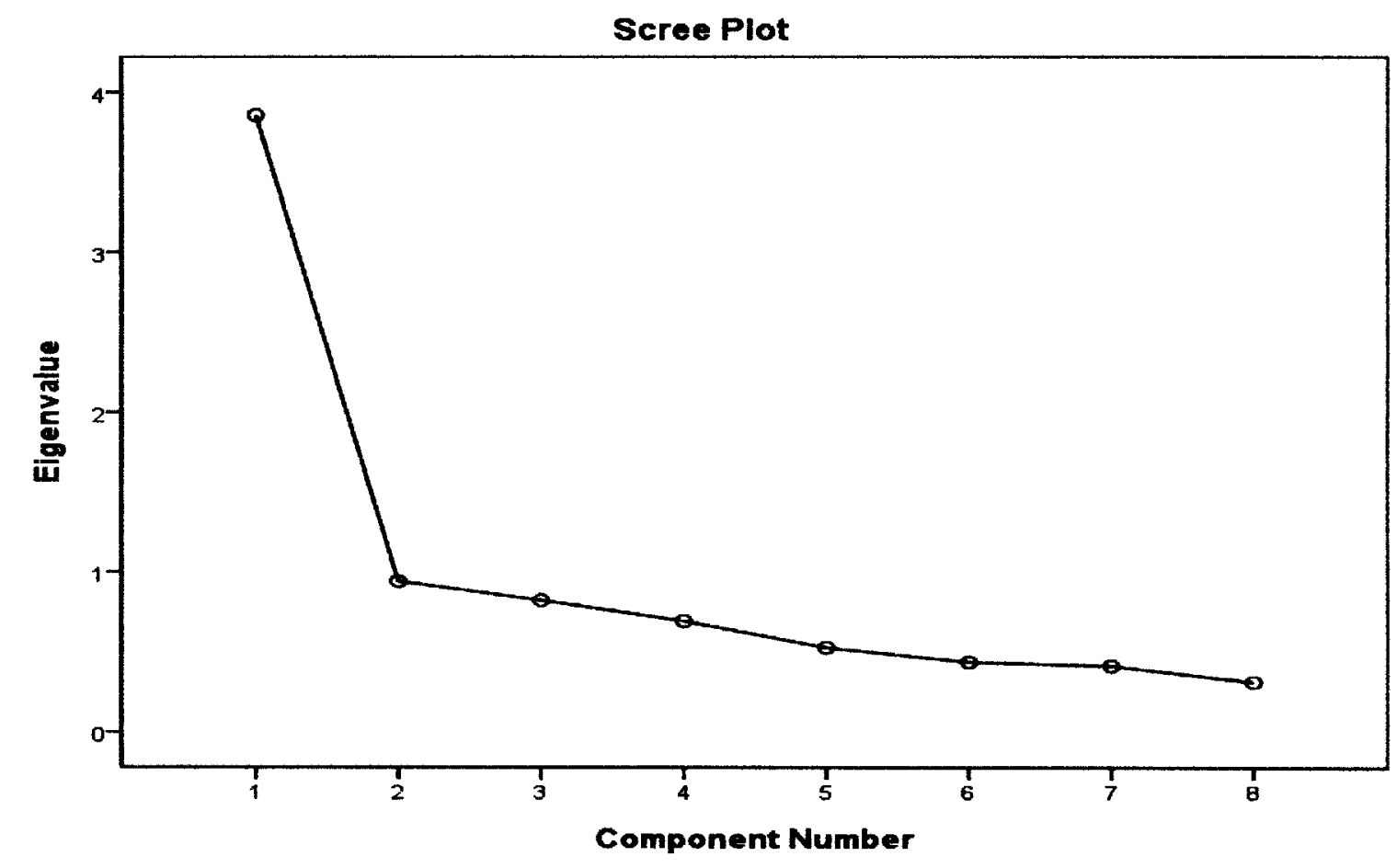

Figure 4. Scree plot for Standard 3, Instruction.

Table 10 lists the eight indicators for the one component solution for Standard 3 with the subsequent factor analysis loading for each. Seven of the eight indicators have strong correlations with the underlying factor (3.1.a, 3.1.c, 3.1.g, 3.1.b, 3.1,e, 3.1.f, and 3.1.d) ranging from .763 for 3.1.a to .647 for 3.1.d. The indicator on homework (3.1.h at $.555)$ has the lowest connection to the overall standard 1 construct. The default setting for SPSS provides a printout of factor loadings only for factors with eigenvalues above one. From Table 9, only Component 1 fits that criterion. It is not possible to determine factor loadings for Component 2 (eigenvalue $=.940)$. Forcing a two-component solution yields a completely different set of loadings. Thus a table parallel to Tables $\mathrm{H} 1$ and $\mathrm{H} 2$ for Standard 3, Instruction, is not possible. 
Table 10

Factor Loadings for Standard 3, Instruction

Indicators $^{\mathrm{a}}$

Loadings

3.1.a There is evidence that effective and varied instructional strategies are used in all classrooms.

3.1.c Instructional strategies/activities are consistently monitored and aligned with the changing needs of a diverse student population to ensure various learning approaches and learning styles are addressed.

3.1.g Teachers examine and discuss student work collaboratively and use this information to inform their practice.

3.1.b Instructional strategies and learning activities are aligned with the district, $\quad .726$ school, and state learning goals and assessment expectations for student learning.

3.1.e There is evidence that teachers incorporate the use of technology in their .683 classrooms.

3.1.f Instructional resources (textbooks, supplemental reading, technology) are .652 sufficient to effectively deliver the curriculum.

3.1.d Teachers demonstrate the content knowledge necessary to challenge and motivate students to high levels of learning.

3.1.h There is evidence that homework is frequent and monitored and tied to .555 instructional practice.

${ }^{\mathrm{a}} \mathrm{KDE}(2004 \mathrm{~b}$, pp. 13-14).

Table 11 presents reliability analysis for the eight indicators for Standard 3 (Instruction). Cronbach's coefficient alpha of .818 for the overall composite demonstrated high internal consistency for the scale, supporting a single instructional construct. Inspection of the alpha with item deleted column indicates that in no instance would removing a single item change the overall scale reliability by very much as these values 
vary only from .809 for 3.1 a to 8.31 for 3.1.h. This provides additional evidence that the eight indicators of the intended standard function as a single factor. The mean and standard deviation for both the individual items and the composite scale $(M=1.67, S D=1.17)$ have acceptable ranges.

Table 11

Internal Reliability and Item Characteristics for Standard 3, Instruction $(N=83)$

$\begin{array}{llll}\text { Indicator } & M & S D & \text { Range }\end{array}$

$\begin{array}{lllll}\text { 3.1.b } & 1.61 & .99 & 5 & .816\end{array}$

$\begin{array}{lllll}3.1 . c & 1.26 & 1.06 & 5 & .810\end{array}$

$\begin{array}{lllll}\text { 3.1.d } & 2.57 & 1.37 & 7 & .822\end{array}$

$\begin{array}{lllll}\text { 3.1.e } & 1.95 & 1.09 & 5 & .818\end{array}$

$\begin{array}{lllll}3.1 . f & 2.10 & 1.53 & 5 & .821\end{array}$

$\begin{array}{lllll}3.1 . \mathrm{g} & 1.19 & 1.08 & 5 & .813\end{array}$

$\begin{array}{lllll}\text { 3.1.h } & 1.36 & 1.19 & 5 & .831\end{array}$

$\begin{array}{lllll}\text { Total } & 1.67 & 1.17 & 5.25 & .818^{\mathrm{b}}\end{array}$

$\bar{a} \alpha-\mathrm{d}=$ alpha with item deleted.

${ }^{b}$ Value for $\alpha-\mathrm{d}$ for Total is Cronbach's coefficient alpha for the entire scale.

As a last additional assessment of construct and external criterion validity (Nitro, 2001), factor-weighted scales were created for each standard, and a correlation matrix was constructed that included all dependent, independent, and control variables. Most correlations are weak; the highest values are only of moderate strength. The approximate range of correlations is .10 to .60 . The highest correlation with the Academic Index (AI) is 
Instruction, $r=.625, p<.01$. Percent FRL was the second highest correlation with the $\mathrm{AI}$, $r=-.561, p<.01$, with a negative influence. Year of Audit was third highest, $r=.532, p<$ .01 . No other variables had coefficients with the AI of .5 or more.

The highest correlations in the table huddle around the standards of this study-Curriculum, Instruction, and Leadership--as they relate to each other and the dependent variable, the Academic Index. Instruction demonstrates the highest association with the Academic Index $(r=.625)$, with Leadership also moderate $(r=.442)$. The intercorrelations among the three standards represent one aspect of external criterion validity. These reflect ideal psychometric properties, i.e., the standards are inter-related, as often happens for components of an overall school reform model, yet are still somewhat independent of one another. These inter-correlations follow: $r=.622$ for Standard 7 (Leadership) with Standard 1 (Curriculum); $r=.629$ for Standard 7 (Leadership) with Standard 3 (Instruction); and, $r=.468$ for Standard 1 (Curriculum) with Standard 3 (Instruction). 
Table 12

Correlation Matrix for Demographic Factors, Leadership, Curriculum, Instruction, and Academic Index $(N=83)$

\begin{tabular}{|c|c|c|c|c|c|c|c|c|c|c|c|c|c|}
\hline & $\mathrm{AI}$ & LEAD & CURR & NST & APP & CTY & $\%$ DIS & $\% \mathrm{FRL}$ & $\%$ GFT & $\%$ LEP & SIZE & $\% \mathrm{WH}$ & YR \\
\hline $\mathrm{AI}$ & $-\cdot$ & $.442^{\prime \prime}$ & $.239^{\circ}$ & $.625^{m+1}$ & $-.331^{\circ}$ & $-.228^{*}$ & -.028 & $-.561^{*}$ & $.451^{\prime \prime}$ & -.173 & .025 & $.256^{\circ}$ & $.532^{41}$ \\
\hline LEAD & & - & $.622^{* *}$ & $.629^{* *}$ & $-299^{* *}$ & -.016 & -.129 & $-.295^{* *}$ & .199 & .178 & .146 & -.161 & .144 \\
\hline CURR & & & -- & $.468^{* *}$ & -.076 & -.158 & -.174 & -.122 & .144 & .094 & .028 & .001 & .076 \\
\hline INST & & & & - & $-.278^{*}$ & -.162 & -.080 & $-.378^{* *}$ & .165 & .068 & .106 & -.027 & $.287^{* *}$ \\
\hline APP & & & & & - & $.310^{* *}$ & -.086 & $.533^{* *}$ & .007 & -.198 & -.007 & $.470^{* *}$ & -.131 \\
\hline CTY & & & & & & - & $-.278^{*}$ & .086 & -.123 & .084 & $.492^{* *}$ & $.240^{*}$ & -.072 \\
\hline$\% \mathrm{DIS}$ & & & & & & & - & .054 & -.034 & -.045 & -.215 & -.181 & -.010 \\
\hline$\% \mathrm{FRL}$ & & & & & & & & - & $-.231^{*}$ & .195 & $-.277^{*}$ & -.173 & .003 \\
\hline$\% \mathrm{GFT}$ & & & & & & & & & -- & -.198 & -.037 & .230 & $.287^{* *}$ \\
\hline \%LEP & & & & & & & & & & -- & .154 & $-.539^{* *}$ & .108 \\
\hline SIZE & & & & & & & & & & & -- & .011 & -.084 \\
\hline$\% \mathrm{WH}$ & & & & & & & & & & & & -- & -.084 \\
\hline YR & & & & & & & & & & & & & -- \\
\hline
\end{tabular}




\section{Research Questions}

This study was guided by four empirical questions. For the convenience of the reader, each research question is stated before the results are presented. Because the psychometric analyses of the sets of indicators for the three standards determined that each is represented by a single factor, factor score weighted scales were used to represent Standards 1, 3, and 7. Research Questions $1-3$ used simultaneous regression; Research Question 4 used hierarchical regression to enter the variables in the same order as listed by Figure 1 and Table 1.

Multiple regression analysis is a statistical method for studying the relation between a dependent variable and two or more independent variables. The purposes are to help the researcher predict the value of some dependent (or criterion) variable from a set of independent (or predictor) variables, to test hypotheses about alternative models of the relation between the variables, or to do some combination of these two things (Shavelson, 1996). The multiple regression provides an index, or regression coefficient, of the relationship between the dependent variable and each of the independent variables. In addition, this method can resolve the degree to which variables are related, as well as approximate the overall effect size. Multiple regressions also have assumptions as to the type and shape of the data, relationships among variables, etc. To use multiple regression to test hypotheses statistically, the following assumptions are made: (a) Independence (scores for a variable are independent of other variable scores); (b) Normality (dependent variable scores are normally distributed for independent variables; (c) Homoscedasticity (variances of dependent and independent variables are equal); and (d) Linearity (dependent and independent variable relationship is linear when independent variables are held constant (Shavelson). The assumption for independence can be calculated by ANOVA; the 
three remaining assumptions can be examined via scatterplots. Or, the four assumptions can be tested within statistical programs such as SPSS. For this study, statistical software, SPSS, was used to calculate results.

In multiple regressions, data from the independent variables can be entered in different orders suggesting different methodologies. One method is simultaneous multiple regression (used in Research Questions 1-3 of this study), in which all independent variables are entered into the analysis at the same time. Then, the effect of each independent variable is assessed on the dependent variable in terms of what it adds to the prediction of the dependent variable (Tabachnick \& Fidell, 1996).

Another method of multiple regression is hierarchical multiple regression (used in Research Question 4) which allows the researcher to examine the influence of several independent variables in a specific order. The researcher can dictate the order in which the variables are entered into the analysis. Individual effects are assessed at the point a given variable is entered (Tabachnick \& Fidell, 1996).

In this research, multiple regression analysis is used to estimate the degree to which the variation of the Academic Index scores (the dependent variable) can be explained by the independent variables: Standard 7 (Leadership), Standard 1 (Curriculum), Standard 3 (Instruction), and Demographic Factors.

\section{Research Question 1}

To what degree do demographic factors affect Standard 7 (Leadership), Standard 1 (Curriculum), Standard 3 (Instruction), and the Academic Index in Kentucky high schools?

The regression results for Research Question 1 are listed in Tables 10-13. Table 10 details the results of simultaneous multiple regression analysis to determine the effects of the Demographic Variables on Standard 7 (Leadership). Tables 13, 14, and 15, give the 
effects of the Demographic Variables on Standard 1 (Curriculum), Standard 3 (Instruction), and the Academic Index, respectively.

In the multiple regression for the effects of the Demographic Variables on Standard 7 (Leadership--Table 13), the independent variables include Appalachian, County vs. Independent, Percent Disability, Percent Free/Reduced Lunch, Percent Gifted, Percent Limited English, School Size (grades 10 \& 11), Percent White, and Year of Audit. Although the model demonstrates a significant relationship between the independent variables and Leadership, $F(9,83)=2.26, p<.001$, none of the individual variables considered yield a significant effect on Leadership. The very small effect size, Adjusted $R^{2}$ $=.12$, is consistent with that finding. In effect, Standard 7 (Leadership) is essentially independent of the demographics of the student population of the high school for this sample. 
Table 13

Regression of Standard 7, Leadership, on the Demographic Variables $(N=83)$

\begin{tabular}{lrrrrr}
\hline Variable & \multicolumn{1}{l}{ B } & \multicolumn{1}{l}{ SE B } & \multicolumn{1}{l}{ Beta } & \multicolumn{1}{l}{ Sig. $t$} \\
\hline Constant & -105.140 & 159.050 & & -.661 & .511 \\
APP & -.108 & .356 & -.054 & -.304 & .762 \\
CTY & .089 & .357 & .033 & .249 & .804 \\
\%DIS & -.017 & .015 & -.122 & -1.109 & .271 \\
\%FRL & -.016 & .010 & -.270 & -1.643 & .105 \\
\%GFT & .023 & .014 & .185 & 1.590 & .116 \\
\%LEP & .062 & .060 & .136 & 1.030 & .306 \\
SIZE & .000 & .001 & .036 & .276 & .784 \\
\%WH & -.010 & .009 & -.184 & -1.110 & .271 \\
YR & .053 & .080 & .076 & .669 & .506 \\
\hline
\end{tabular}

Table 14 presents the results of Standard 1, Curriculum, regressed on the Demographic Variables $F(1,83)=51.15, p<.001$. Looking at the Adjusted $R^{2}$, very little of the variance in Standard 1, Curriculum, is explained by the Demographic Variables (adj. $\left.R^{2}=.008\right)$. In fact, no demographic variable significantly influenced curriculum when controlling for other variables in the model, although County/Independent and Percent Disabled come close at $p=054, p=.085$, respectively with the negative direction of the coefficients indicating that for Independent Districts, quality of leadership is less and for $\%$ DIS that higher percentages of students with disabilities are associated with less quality in curriculum. This means Standard 1 is essentially independent of the demographic factors 
examined.

Table 14

Regression of Standard 1, Curriculum, on the Demographic Variables $(N=83)$

\begin{tabular}{lccccc}
\hline Variable & $B$ & $S E B$ & Beta & $t$ & Sig. $t$ \\
\hline Constant & -44.860 & 169.160 & & -.265 & .792 \\
APP & .129 & .378 & .064 & .342 & .733 \\
CTY & -.744 & .379 & -.280 & -1.962 & .054 \\
\%DIS & -.028 & .016 & -.204 & -1.743 & .085 \\
\%FRL & -.007 & .010 & -.114 & -.650 & .518 \\
\%GFT & .007 & .015 & .060 & .483 & .630 \\
\%LEP & .079 & .064 & .173 & 1.232 & .222 \\
SIZE & .000 & .001 & .075 & .540 & .591 \\
\%WH & .003 & .010 & .060 & .340 & .735 \\
YR & .023 & .085 & .032 & .268 & .789 \\
\hline
\end{tabular}

Table 15 displays the analysis to determine the effects of Demographic Variables on Standard 3, Instruction. Of the variables considered, only Percent Free/Reduced Lunch and Year of the Audit yield a significant effect. The ANOVA for the model is significant, $F(9,83)=3.014, p<.001$. The Adjusted $R^{2}$ of .181 indicates a small effect for the Demographic Variables on Instruction. Looking at the standardized coefficients, the variable with the strongest effect on instruction was Percent Free/Reduced Lunch, which had a beta of -.395 , indicating a negative impact on Standard 3, Instruction. Standard 3, 
Instruction, would increase nearly two-fifths of a standard deviation if the number of students on Free/Reduced Lunch decreased by one standard deviation. The beta for Year of the Audit (.281) also indicates a substantial effect on Standard 3, Instruction, almost threetenths of a standard deviation. For this variable, coded by the year the audit was conducted (from 2001-2005), later years are associated with better quality instruction.

Table 15

Regression of Standard 3, Instruction, on the Demographic Variables $(N=83)$

\begin{tabular}{lrrrrr}
\hline Variable & \multicolumn{2}{c}{ SE $B$} & Beta & \multicolumn{1}{c}{$t$} & Sig. $t$ \\
\hline Constant & -391.910 & 153.542 & & -2.552 & .013 \\
APP & .133 & .343 & .066 & .386 & .700 \\
CTY & -.540 & .344 & -.203 & -1.568 & .121 \\
\%DIS & -.012 & .015 & -.087 & -.818 & .416 \\
\%FRL & -.023 & .009 & -.395 & -2.488 & .015 \\
\%GFT & .000 & .014 & .001 & -.011 & .991 \\
\%LEP & .047 & .058 & .103 & .809 & .421 \\
SIZE & .001 & .001 & .106 & .839 & .404 \\
\%WH & -.002 & .009 & -.042 & -.261 & .795 \\
YR & .196 & .077 & .281 & 2.558 & .013 \\
\hline
\end{tabular}

The multiple regression for the Academic Index on the Demographic Variables is shown in Table 16. The ANOVA is significant, $F(9,83)=17.615, p<.001$. The Adjusted $R^{2}$ of .65 indicates a very strong effect on the Academic Index; almost two-thirds of the variation in the Academic Index is explained by the demographic composition of the 
student population of a school. Several Demographic Variables produced significant effects: County/Independent districts, Percent Free/Reduced Lunch, Percent Gifted, Percent White and Year of Audit. Controlling for the other variables in the model, Percent Free/Reduced Lunch and Year of Audit have the strongest effects with standardized betas of -.38 , and .46 , respectively. This is interpreted as a gain of almost half a deviation unit associated with a one standard deviation increase in Year of Audit; for Percent Free/Reduced Lunch, this is interpreted as a loss of .38 standard deviation units on the AI associated with a one standard deviation unit increase in percentage of free/reduced lunch participants. For this data base, the coding for County indicates that independent schools scored higher than county schools. The strong beta for Year of Audit suggests that high schools across the state demonstrating a significant level of increase in student achievement for the five years of data collection. 
Table 16

Regression of Academic Index on the Demographic Variables $(N=83)$

\begin{tabular}{lccccc}
\hline Variable & $B$ & $S E B$ & Beta & $t$ & Sig. $t$ \\
\hline Constant & -6338.978 & 993.917 & & -6.378 & $<.001$ \\
APP & -2.509 & 2.223 & -.126 & -1.128 & .263 \\
CTY & -5.425 & 2.228 & -.208 & -2.435 & .017 \\
\%DIS & -.004 & .095 & -.003 & -.041 & .968 \\
\%FRL & -.221 & .061 & -.380 & -3.635 & $<.001$ \\
\%GFT & .179 & .089 & .149 & 2.012 & .048 \\
\%LEP & $<.001$ & .377 & $<.001$ & $<.001$ & 1.000 \\
SIZE & .005 & .005 & .081 & .981 & .330 \\
\%WH & .145 & .059 & .259 & 2.463 & .016 \\
YR & 3.195 & .497 & .463 & 6.426 & $<.001$ \\
\hline
\end{tabular}

Research Question 2

To what degree does Standard 7 (Leadership) affect Standard 1 (Curriculum), Standard 3 (Instruction), and the Academic Index in Kentucky high schools?

The results for Research Question 2 are displayed in Tables 17, 18, and 19. Table 17 explains the results of the simultaneous multiple regression analysis for Standard 7, Leadership, on Standard 1, Curriculum. Standard 7, Leadership, yields a significant effect on Standard 1, Curriculum, $F(1,83)=51.147, p<.001$, explaining $40 \%$ of the variation in Standard 1, Curriculum, a modest effect. The raw/unstandardized coefficient, $B$, indicates that a one unit increase in Standard 7, Leadership, would produce a .623 unit increase in 
the dependent variable (Curriculum).

Table 17

Regression of Standard 1, Curriculum, on Standard 7, Leadership $(N=83)$

\begin{tabular}{lccccc}
\hline Variable & $B$ & $S E B$ & Beta & $t$ & Sig. $t$ \\
\hline Constant & $2.007 \mathrm{E}-16$ & .086 & & $<.001$ & 1.000 \\
Leadership & .623 & .087 & .622 & 7.152 & $<.001$ \\
\hline
\end{tabular}

Table 15 presents the results of the simultaneous regression for the effects of Standard 7, Leadership, on Standard 3, Instruction. The equation is significant, $F(1,83)=$ $53.096, p<.001$, with an effect size of .39 . The raw unstandardized coefficient, $B$, indicates that a one unit increase in Standard 7, Leadership, would produce a .63 unit increase in the dependent variable (Instruction).

Table 18

Regression of Standard 3, Instruction, on Standard 7, Leadership $(N=83)$

\begin{tabular}{lccccc}
\hline Variable & $B$ & $S E B$ & Beta & $t$ & Sig. $t$ \\
\hline Constant & $-3.333 \mathrm{E}-16$ & .086 & & $<.001$ & 1.000 \\
Leadership & .629 & .086 & .629 & 7.287 & $<.001$ \\
\hline
\end{tabular}

The regression of the Academic Index on Standard 7, Leadership, is significant, $F(1,83)=19.61, p<.001$ (see Table 16). The effect size of .20 accounts directly for onefifth (20\%) of the variation in the Academic Index. The standardized beta of .44 specifies that a one unit increase in the standard deviation for Standard 7, Leadership, would 
generate a .44 change in standard deviation units in the Academic Index.

Table 19

Regression of the Academic Index, on Standard 7, Leadership $(N=83)$

\begin{tabular}{lrrrrr}
\hline Variable & $B$ & $S E B$ & Beta & \multicolumn{1}{c}{$t$} & Sig. $t$ \\
\hline Constant & 58.854 & .975 & & 60.362 & $<.001$ \\
Leadership & 4.346 & .981 & .442 & 4.429 & $<.001$ \\
\hline
\end{tabular}

Research Question 3

To what degree do Standard 1 (Curriculum) and Standard 3 (Instruction) affect the Academic Index in Kentucky high schools?

Table 20 exhibits the influence of Standard 1(Curriculum) and Standard 3

(Instruction) on the Academic Index. The ANOVA indicates the model is significant, $F(2$, $83)=26.01, p<.001$, explaining $39 \%$ of the variance in the Academic Index, the dependent variable. The entire effect of the model comes from Standard 3, Instruction; Standard 1, Curriculum, has no impact on the Academic Index. A one unit increase in Instruction would generate a 6.47 unit gain in the Academic Index, the dependent variable. As depicted by this regression analysis, Standard 3, Instruction, has the sole influence on the Academic Index with Standard 1, Curriculum, not significant. 
Table 20

Regression of the Academic Index on Standard 1, Curriculum, and Standard 3, Instruction $(N=83)$

\begin{tabular}{lccccr}
\hline Variable & $B$ & $S E B$ & Beta & $t$ & Sig. $t$ \\
\hline Constant & 58.854 & .851 & & 69.146 & $<.001$ \\
Curriculum & -.672 & .969 & -.068 & -.694 & .490 \\
Instruction & 6.466 & .970 & .657 & 6.668 & $<.001$ \\
\hline
\end{tabular}

\section{Research Question 4}

To what degree do Standard 1 (Curriculum) and Standard 3 (Instruction) mediate the effect of Standard 7 (Leadership) on the Academic Index, controlling for demographics in Kentucky high schools?

Table 21 incorporates all the independent variables, exhibiting the hierarchical multiple regression to establish the effects of Demographic Factors, Standard 7 (Leadership), and Standards 1 (Curriculum) and 3 (Instruction) on the Academic Index. All Demographic Factors were included. Each of the ANOVAs for the model for the three separate steps of Table 18 is significant. Separate calculations, the full and reduced model $F$-test, were completed by computing the residual sums of square of the models. This test indicates whether adding new variables improved the model when going from Step 1 (Demographics only) to Step 2 (adding Leadership) and from Step 2 to Step 3 (adding Curriculum and Instruction). The model was improved with each subsequent Step (equations not reported). The change in the $R^{2}$ value is reported after Step 3 of the hierarchical regression.

For Step 1, the ANOVA was significant, $F(9,83)=17.615, p<.001$. The Adjusted 
$R^{2}$ of .65 indicates a large effect on the Academic Index. The demographic controls County/Independent district, Percent Gifted, Percent Free/Reduced Lunch, Percent White, and Year of Audit are all statistically significant at the $p<.05$ level.

For Step 2, Standard 7 (Leadership) was added to the equation to determine its effect after demographic factors were controlled, with the ANOVA for the model significant, $F(10,83)=21.329, p<.001$. This produced an increment in the Adjusted $R^{2}$ from .65 to .71 . The demographic controls of County/Independent district, Percent Free/Reduced Lunch, Percent White, and Year of Audit continue to be statistically significant but Percent Gifted loses significance. Leadership is also significant with a standardized beta of .284 .

For Step 3, Standard 1 (Curriculum) and Standard 3 (Instruction) were added to consider the extent that Standard 7 (Leadership) mediates Academic Performance when the Demographic Factors are controlled. The significant results for the Step 3 model, $F(12,83)$ $=22.019, p<.001$, produced an additional increment, from effect size of .71 to .76 .

Standard 3 (Instruction) was significant although Standard 1 (Curriculum) added nothing for Step 3. The other significant variables from Step 2 variables remained significant for Step 3. Explaining $76 \%$ of the variance in the final model is an unusually high effect in educational research.

Standardized beta results signify the increase in standard deviation units on the Academic Index that a one standard deviation increase in the various independent variables would produce. For this final hierarchical model (Step 3), the demographic factors producing a statistically significant effect are noted with the first listed displaying the most significance-- Year of Audit, Percent White, Percent Free/Reduced Lunch, and County/Independent district--ranging from $\beta=.442$ to $\beta=-.217$. The negative beta for 
Percent Free/Reduced Lunch is expected (influence of increased poverty on achievement). The negative beta for County/Independent districts indicates that in this data set, the schools from independent districts scored higher. Both Standard 7 (Leadership) from Step $2(\beta=.215)$ and Standard 3 (Instruction) from Step $3(\beta=.279)$ are significant even after accounting for the demographic factors. Notable is the fact that the beta score for Instruction exceeds that of Percent Free/Reduced Lunch, an indicator of poverty, while the direct influence of Leadership is almost as high. This validates that these professionally alterable school practices can have a significant impact, even when demographics loom large. This final regression confirms the mediated effects model of leadership, with indirect influence on the Academic Index through instruction but not curriculum. For this model, Standard 7 (Leadership) has both a direct and a mediated effect on the Academic Index. 
Table 21

Regression of the Academic Index on Standard 7, Leadership, Controlling for Demographic

Factors and as Mediated by Standard 1, Curriculum, and Standard 3, Instruction $(N=83)$

\begin{tabular}{|c|c|c|c|c|c|}
\hline Variable & $B$ & $S E B$ & Beta & $t$ & Sig. $t$ \\
\hline \multicolumn{6}{|c|}{ Step 1} \\
\hline Constant & -6338.978 & 993.917 & & -6.378 & $<.001$ \\
\hline APP & -2.509 & 2.223 & -.126 & -1.128 & .263 \\
\hline CTY & -5.425 & 2.228 & -.208 & -2.435 & .017 \\
\hline$\%$ DIS & -.004 & .095 & -.003 & -.041 & .968 \\
\hline$\% \mathrm{FRL}$ & -.221 & .061 & -.380 & -3.635 & $<.001$ \\
\hline$\% \mathrm{GFT}$ & .179 & .089 & .149 & 2.012 & .048 \\
\hline$\%$ LEP & $<.001$ & .377 & $<.001$ & $<.001$ & 1.000 \\
\hline SIZE & .005 & .005 & .081 & .981 & .330 \\
\hline$\% \mathrm{WH}$ & .145 & .059 & .259 & 2.463 & .016 \\
\hline YR & 3.195 & .497 & .463 & 6.426 & $<.001$ \\
\hline
\end{tabular}


Table 21. (continued)

\begin{tabular}{lccccc}
\hline Variable & \multicolumn{1}{c}{ SE B } & Beta & \multicolumn{1}{l}{ Sig. $t$} \\
\hline & & Step 2 & & \\
Constant & -6045.470 & 898.068 & & -6.732 & $<.001$ \\
APP & -2.207 & 2.004 & -.111 & -1.101 & .275 \\
CTY & -5.672 & 2.008 & -.217 & -2.825 & .006 \\
\%DIS & .043 & .086 & .032 & .501 & .618 \\
\%FRL & -.177 & .056 & -.303 & -3.162 & .002 \\
\%GFT & .116 & .081 & .096 & 1.420 & .160 \\
\%LEP &. .174 & .342 & -.039 & -.507 & .613 \\
SIZE & .004 & .004 & .071 & .952 & .344 \\
\%WH & .174 & .054 & .311 & 3.257 & .002 \\
YR & 3.046 & .449 & .442 & 6.781 & $<.001$ \\
Leadership & 2.792 & .659 & .284 & 4.237 & $<.001$ \\
\hline & & & & $($ table continues)
\end{tabular}


Table 21. (continued)

\begin{tabular}{|c|c|c|c|c|c|}
\hline Variable & $B$ & $S E B$ & Beta & $t$ & Sig. $t$ \\
\hline \multicolumn{6}{|c|}{ Step 3} \\
\hline Constant & -5098.133 & 869.948 & & -5.860 & $<.001$ \\
\hline APP & -2.479 & 1.863 & -.125 & -1.331 & .188 \\
\hline CTY & -5.078 & 1.989 & -.194 & -2.553 & .013 \\
\hline$\% \mathrm{DIS}$ & .029 & .080 & .021 & .358 & .721 \\
\hline$\% \mathrm{FRL}$ & -.132 & .053 & -.226 & -2.488 & .015 \\
\hline$\%$ GFT & .140 & .076 & .117 & 1.849 & .069 \\
\hline$\%$ LEP & -.160 & .317 & -.036 & -.506 & .615 \\
\hline SIZE & .003 & .004 & .054 & .775 & .441 \\
\hline$\% \mathrm{WH}$ & .178 & .050 & .318 & 3.555 & .001 \\
\hline YR & 2.572 & .435 & .373 & 5.909 & $<.001$ \\
\hline Leadership & 2.118 & .878 & .215 & 2.413 & .018 \\
\hline Curriculum & -1.273 & .745 & -.129 & -1.708 . & .092 \\
\hline Instruction & 2.744 & .777 & .279 & 3.530 & $<.001$ \\
\hline
\end{tabular}

Note. Adjusted $R^{2}=.65$ for Step $1 ; \Delta$ in Adjusted $R^{2}=.06$ for Step 2; $\Delta$ in Adjusted $R^{2}=$ .05 for Step $3(p s<.001)$.

\section{Summary}

The purpose of this study was: (a) to examine the effects of school level leadership on curriculum and instruction as they pertain to student success depicted by the Academic Index scores in Kentucky high schools, and (b) to investigate relationships, if any, that may 
exist among certain demographic factors such as school size, school setting, gender, and student achievement. To explore the relationships, this study used secondary data from Kentucky's Scholastic Audits performed by the KDE audit-trained teams over a five-year period (2001-2005). The study was limited to data provided by the Kentucky high schools that completed an audit or review during the specified time frame period. The Scholastic Audit process is based on the Standards and Indicators for School Improvement (SISI), a document created by KDE to guide school improvement in all Kentucky schools. The SISI contains nine standards with 88 indicators. This study focused on Standard 1 (Curriculum), Standard 3 (Instruction), and Standard 7 (Leadership), controlling for Demographic Factors obtained from the Kentucky Performance Report for each school of the study. The sample for this study was 83 high schools from the total population of 224 Kentucky high schools, grades 9-12.

The statistical procedures used in the study included descriptive statistics, psychometric analysis, and multiple regressions. Both simultaneous and hierarchical regressions were conducted. No missing or out-of-range data were found. The dependent variable for the study was the Academic Index, a $90.25 \%$ composite of the overall Accountability Index--a score containing of KCCT content areas results generated in tenth and eleventh grades for the schools in this study. Three of the nine standards from the Standards and Indictors for School Improvement (SISI) document have been designated as independent variables: Standard 7 (Leadership), Standard 1 (Curriculum), and Standard 3 (Instruction). Demographic Factors were identified as control variables. Those used in this study included: Appalachian, County/Independent district, Percent Disabled, Percent Free/Reduced Lunch, Percent Gifted, Percent Limited English Proficiency, School Size, Percent White, and Year of Audit. 
Factor analysis was computed on the three sets of indicators of the study: Standard 7, Standard 1, and Standard 3. In each case, a single factor emerged, explaining $45.4 \%$, $52.9 \%$, and $48.2 \%$, respectively, of the variance among the indicator sets. To examine the internal reliability of the computations resulting from the FA, Cronbach's alpha was computed. Cronbach's coefficient alpha calculated on the single factors yielded overall values of $.873, .844$, and .837 , respectively, for Standard 7 (Leadership), Standard 1 (Curriculum), and Standard 3 (Instruction). Inter-scale correlations among these three standards further confirmed their external criterion validity, with $r$ ranging from .239 to .625. These results represent exceptional psychometric properties for these three standards and support the conceptualization and development by KDE of the respective indicators for each standard.

Multiple regression analyses tested the hypothetical study model as shown in Figure 1, page 16. The demographic factors substantially affect on the Academic Index as they explain $65 \%$ of the variation in the Academic Index by themselves. Standard 7 (Leadership) explains 39\% variation in Standard 1 (Curriculum) as well as $40 \%$ variance in Standard 3 (Instruction). Standard 7 (Leadership) has a smaller direct effect on the Academic Index, explaining 20\% of the variance. Together, Standards 1 and 3 determine $40 \%$ variance in the Academic Index; however, all of this comes from Standard 3 (Instruction) as Standard 1 (Curriculum) is not significant. When the final hierarchical regression was computed, the influence of Standard 7 (Leadership) and Standard 3 (Instruction) along with four of the demographic factors--Year of Audit, Percent White, Percent Free/Reduced Lunch, and County/Independent school district--had an overall effect size of .76, exceptionally high as educational research goes.

The central research question captured the purpose of this study: Based on 
Scholastic Audits, what are the effects of instructional leadership in Kentucky accountability scores, as mediated by curriculum and instruction? The final regression confirmed the mediated effects model of leadership, with an indirect effect of Standard 7 (Leadership) on the Academic Index through its effect on Instruction. In addition Standard 7 has a $21.5 \%$ direct influence on the Academic Index. Thus, the results of this study reiterate the work of Murphy (2004) and others, that a mediated effects model most accurately describes the dual influence of the high school principal on student achievement, both directly as well as indirectly through impact on instruction. 


\title{
CHAPTER V \\ DISCUSSION AND CONCLUSIONS
}

\author{
Introduction
}

A Nation at Risk (National Commission on Excellence in Education, 1983) seared American schools, riveting public scrutiny as the report illuminated the need for improved achievement, mandated assessment, and increased accountability. Resulting waves of educational reforms ensued throughout the following decades. Kentucky had its own initiatives sparked by lawsuit challenging the state's funding formula. The lawsuit alleged that funding relied too heavily on local resources, creating an inequitable educational opportunity for the children in property-poor districts. The Kentucky Supreme Court's response went beyond normal precedence in finance equity suits, ruling the state's entire educational system unconstitutional. In effect, this ruling took on landmark significance (Steffy, 1993) as the Commonwealth was forced to pass what is heralded as the most comprehensive education package ever passed by a state legislative body (Steffy)--the Kentucky Education Reform Act of 1990 (KERA).

The extensive reform initiatives in Kentucky involved policies designed to: equalize educational funding and create equity for all students, particularly for propertypoor school districts; implement shared decision making at the school level; and require all schools to improve academically over time. The state created an accountability model, Kentucky Instructional Results Information System (KIRIS) that was later replaced by the Commonwealth Accountability Testing System (CATS) which had better reliability but 
retained the essential features of KIRIS, including the requirement that all schools move toward the statewide adopted goal of Proficiency, 100 on a 140-point Accountability Index. To assist schools in reaching this challenging goal, the Kentucky Department of Education (KDE) developed the Standards and Indicators for School Improvement (SISI) (KDE, 2003) and the Scholastic Audit.

The SISI document represented an extension of standards-based accountability from content areas to whole school reform. It consists of nine standards and 88 indicators divided into three areas: Academic Performance, Learning Environment, and Efficiency. Among these standards are: Leadership (Standard 7, with a focus on instructional improvement and increased student achievement), Curriculum (Standard 1), and Instruction (Standard 3), the three standards of particular emphasis for this study. Tying these together is the leadership of principals, a key to schools reaching the proficiency level by the year 2014 .

Expectations for school level leadership are examined in the context of school accountability, based on Scholastic Audits. As defined by the Accountability Index schools, every two years schools are labeled as meeting their goal, progressing, or needing assistance. All schools needing assistance are further divided into three classifications: (a) Level 3 (the lowest one-third that receive a mandated Scholastic Audit), (b) Level 2 (the middle third that receive a Scholastic Review), and (c) Level 1 (the top one-third of which most conduct a self-review). The Scholastic Audit required through KRS.6455 uses the SISI document as the evaluative instrument; audits are conducted by KDE-trained teams. With the regimented audit process of observations, interviews, and review of school documents, the teams assign a level (one of four) to each of the $\mathbf{8 8}$ indicators across all nine standards. After assimilation of results, $\mathrm{KDE}$ makes recommendations to guide school 
improvement efforts. Commendations are also offered when the audit team determines an exemplary score is achieved for a standard.

KDE's adoption of the SISI document has played a key role in changing the perspectives on principal leadership around the state. Schools are expected to make ongoing improvement toward established goals, meaning that principals must continuously seek innovative means of inspiring both teachers and the wider educational community to augment increased student achievement. Kentucky principals are also accountable for meeting the broad range of high-stakes value-added accountability expectations in the Standards for School Leaders (Interstate School Leaders Licensure Consortium [ISLLC], 1994), and federal mandates such as No Child Left Behind (NCLB)--all directed toward school improvement. While Kentucky principals must be mindful of multiple sets of standards, the most important aspect of achievement expectations is the level of student success on the Commonwealth Accountability Testing System (CATS). Principals are held responsible for student success and are, perhaps, the most influential entity in the school building; however, there is a lack of information on the specific pathways by which principal leadership affects academic success, especially at the high school level. It is even more the case in Kentucky where most studies of educational accountability have been conducted at the elementary level (cf. Petrokso, 2000).

One source of supporting documentation on the impact of the SISI document as a framework for school improvement is the KDE Scholastic Audit database. These secondary data were collected during a five-year period on both successful and struggling schools and provide an in-depth view of a school. Before this study, KDE has conducted only limited analyses of the audit data. At present, three dissertation studies have been completed that investigated the impact of the Scholastic Audit and achievement outcomes 
for specific standards in Kentucky: B. C. Ennis (2007) on Leadership and Professional Growth, Development and Evaluation; McKinney (2007) on Leadership, Curriculum, and Instruction; and Saravia (2008) on School Culture and Parent Involvement. All these investigations were at the elementary level. One other study of the Scholastic Audit is in progress at the elementary level. To date, no work on the Scholastic Audit has been conducted at the high school level. This study partially replicates the McKinney (2007) study, but at the high school level: Standard 1 (Curriculum) and Standard 3 (Instruction) are examined for the extent that they mediate the effects of Standard 7 (Leadership) on student achievement, controlling for demographic background.

The central research question for this study is: Based on Scholastic Audits, what are the effects of instructional leadership on Kentucky accountability scores, as mediated by curriculum and instruction? This study is limited to Kentucky high schools. The information for this study represents the KDE secondary database obtained from KDE Scholastic Audits and supplemented by school demographic and Academic Index information obtained from the Kentucky Performance Report.

The remainder of this chapter includes a brief overview of the study, discussion and analysis of the findings, recommendations, and conclusions.

The Study in Brief

This quantitative research study analyzed secondary data from the Kentucky Department of Education (KDE): (a) to examine the effects of school level leadership on curriculum and instruction as they pertain to student success depicted by the Academic Index scores in Kentucky high schools, and (b) to investigate relationships, if any, that may exist among certain demographic factors such as school size, school setting, gender, and student achievement. The data were compiled through Scholastic Audits of both successful 
and struggling schools in Kentucky during 2001-2005. This process became law in 1998 when the Kentucky legislative body passed KRS 158.6455 trying to insure that all students in Kentucky schools could be successful. The Scholastic Audit is used by KDE to provide an intense review of school effectiveness and to determine why they are not meeting their established base line goals. The SISI document is the basic assessment and evaluative instrument used in the Scholastic Audits. Each of the 88 indicators is scored on a fourpoint behaviorally defined scale ( 4 high) depicting degree of implementation. For this study, the four-point scale was converted into a $0,2,5,7$ scale to reflect the more difficult jump from level 2 to level 3 (McKinney, 2007). Those audit scores as converted represent the implementation for each standard.

The school was the unit of analysis ( $N=83 \mathrm{Kentucky} \mathrm{high} \mathrm{schools)} \mathrm{for} \mathrm{collection}$ of data by KDE and for this study, with the objective of determining the relationships among leadership, curriculum, instruction, and accountability scores, along with demographic factors. Current literature suggests principal leadership and school effectiveness are affected by the school and community socioeconomic factors (e.g., Andrews \& Soder, 1987; Hallinger \& Murphy, 1986; Roeder, 2001; Smith, 2005, 2006). This element is addressed in the study, as demographic factors are incorporated into the Scholastic Audit database for their effects on Leadership (principals), Curriculum, and Instruction with respect to the CATS accountability scores. The demographic factors, identified as independent variables for this study, were gathered from the Kentucky Performance Report. Demographic controls included are: Appalachian, County versus Independent School District, Percent Disabled, Percent Free/Reduced Lunch, Percent Gifted, Percent Limited English Proficiency, School Size, Percent White, and Year of Audit. Descriptive statistics were reported for the Demographic Factors. 
The Academic Index scores, obtained from the KPR, were identified as the dependent variable. Three of the nine standards from the Standards and Indicators for School Improvement (SISI) document (KDE, 2004a) have been designated as independent variables: Standard 7 (Leadership), Standard 1 Curriculum), and Standard 3 (Instruction). Consistent with Figure 1, (p. 16), Standard 7 is the target alterable variable (Bloom, 1980); Standards 1 and 3 are designated as mediating factors through which the leadership operates. Scores for these standards were obtained from the Scholastic Audits.

Because no formal psychometric analysis had been done by KDE on the 88 indicators of the nine standards, exploratory factor analyses were conducted to determine whether the indicators representing Standard 1 (Curriculum) would load into a single factor. Eigenvalues and scree plots from those principal component analyses provided additional information on the existence of the underlying factor structure. Factor analysis was also conducted to determine if the indicators of Standard 3 (Instruction) and Standard 7 (Leadership) could be considered a single factor. Cronbach's alpha was used to determine the internal scale validity of these resulting factors. Inter-scale correlations, taken from the full correlation matrix for the entire study, were examined as one form of external criterion validity, i.e., interdependence among the three standards.

The primary form of analysis was multiple regressions. In regression, an equation with several independent variables is estimated attempting to isolate the separate effect each has on the dependent variable (Smith, 1985, p. 515). Multiple regressions were conducted to answer the research questions aligned with the relationships laid out in Figure 1. Simultaneous multiple regression was used to compute equations for Research Questions 1-3. A three-step hierarchical multiple regression analysis was used for Research Question 4 to explore the relationships among the variables in the full model. In Step 1, the 
demographic factors were entered. In Step 2, Standard 7 (Leadership) was entered. Finally, the Mediating Factors (Standard 1, Curriculum and Standard 3, Instruction) were added in Step 3. This hierarchical regression provides a test of the mediated effect of leadership (through efforts in curriculum and instruction) on achievement, net of socio-demographic influence.

This study was presented to and approved by the Institutional Review Boards at both the University of Louisville and Western Kentucky University. Documentation is included in Appendix G.

\section{Discussion}

The topics in this section follow the Chapter IV sequence--Descriptive Statistics, Psychometric Analysis, and the Research Questions. The findings are briefly recapitulated and then analyzed in the context of how this study has contributed to the empirical research base.

Initially, available research-based literature on leadership seemed quite extensive; however, there is apparently lack of empirical research that connects high school leadership to Kentucky's high-stakes accountability--that is, connecting principal leadership in Kentucky to student outcomes. Educational leadership literature before the 1980s was heavily classroom focused; it featured practices intended to influence classroom curriculum and instruction directly (Beck \& Murphy, 1993). The 1980s brought a new surge of interest into educational leadership effectiveness inclusive of the effects of principal leadership (Hallinger \& Heck, 1998). These studies, however, focused more on the direct and indirect district level posture shaping principalship practices. The reform initiatives of the 1990s emphasized the principal's responsibility for instructional leadership as opposed to the district superintendent. The emergence of the principal as the 
school's instructional leader is directly attributable to the effective schools research (Hallinger \& Murphy, 1986; Lezotte \& Bancroft, 1985) which found that instructionally effective schools are characterized by strong instructional leadership typically present within the school building.

The researcher found few studies that examined the means by which instructional leadership behavior at the school level may affect school procedures and student outcomes. Heck, Larson, and Marcoulides (1990) examined all public schools (P-12) in the state of California. Two additional studies (Dwyer et al., 1983; Dwyer, 1986) connecting the principal's contribution to instruction were found; the focus of both studies was elementary schools. Owings, Kaplan, and Nunnery (2005) found that a significant relationship exists between principals' quality at certain grade levels and student achievement on the Virginia Standards of Learning tests. The statewide study found that principals rated higher on school leadership as measured by ILSSC. These schools have higher student achievement than comparable schools headed by lower rated principals controlling for socioeconomic status.

Most research useful to the recent reforms in Kentucky focus on a curricular issue such as middle school science (L. S. Ennis, 2002), achievement trends (cf. Education Trust, 2001; Poggio, 2000; Prichard Committee for Academic Excellence, 1999), or effects of socioeconomic factors on school academic outcomes (e.g., Guskey, 1997; Harwell \& LeBeau, 2010; Lyons, 2004; S. K. Miller \& Moore, 2006; Moore, 2003; Roeder, 1999, 2000, 2001; Smith, 2005, 2006). Washington (2002) examined feminine leadership in her study of Kentucky female superintendents, but did not directly apply the qualities of female leadership to student success. Blackmore (1989), Fennell (2005), Owen (1986) and Rosener (1990) studied women in leadership roles as well but did not connect leadership 
qualities to student outcomes and were not specific to Kentucky. Thus, the research clearly indicates the need for more focused investigation of the effects of leadership on achievement outcomes, particularly under the high-stakes accountability conditions in Kentucky (McKinney, 2007). This study provides just that, examining leadership in the context of a mediated effects model, based on Kentucky's Scholastic Audits data set for Kentucky high schools. Because the audits are derived from Kentucky's Standards and Indicators for School Improvement and conducted in Kentucky high schools, this research is unique to the state.

\section{Descriptive Statistics}

Descriptive statistics were reported for the demographic factors, school audit data, and the school Academic Index, the dependent variable. Data were taken from Kentucky high schools, grades 10-11. The demographic factors included: Appalachian, County versus Independent districts, Percent Disabled, Percent Free/Reduced Lunch, Percent Gifted, Percent Limited English, School Size (number of students in grades 10-11 in audited schools), Percent White, and Year of Audit. Descriptions for the Standard 7 (Leadership), Standard 1 (Curriculum), and Standard 3 (Instruction) are included in the section below.

Analysis

Results indicated that $87 \%$ of the students in audited schools were White compared with the state average of $86.5 \%$ (KDE, 2006e) White in all Kentucky schools. The percent of participation in Free/Reduced Lunch for the sample was $42 \%$ compared with the state average of $39 \%$. A factor to this high average of participation is likely because lowperforming schools constitute the larger portion of audited schools. Although notable exceptions exist, many low-performing schools in Kentucky, as well as the nation, are also 
high poverty schools (Kannapel \& Clements, 2005). Therefore, it is realistic that the results in this study would illustrate a tendency for these schools toward greater socioeconomic disadvantage. Noting that all Kentucky schools are required to score proficiency (100 on the Accountability Index) by the year 2014 is important. Even the schools that began with an initial lower baseline score, perhaps due to socioeconomics, must score 100 by 2014 just as the schools starting with much higher baseline scores.

The percentage of Gifted students (13\%) is below the statewide average of $17.5 \%$ (KDE, 2006e). This represents an additional disadvantage for struggling schools as they often have fewer gifted students. Forty-one percent of the schools are in the Appalachian region while the proportion of students with disabilities in the study is approximately equal to the statewide averages. Percent Disabled is $11.5 \%$ for the audited schools as compared with the statewide average of $11 \%$. The percent of Limited English was identified as approximately $11.5 \%$ compared with $1.0 \%$ (KDE, 2006e) throughout the state. In this study, $80 \%$ of the schools were County school districts compared with $20 \%$ from Independent school districts.

\section{Psychometric Analysis}

For this study, psychometric analysis consisted of the application of factor analysis, Cronbach's (1951) coefficient alpha, and inter-scale correlations. Exploratory factor analysis was conducted on the set of indicators for each of the three standards (7, Leadership; 1, Curriculum; and, 3, Instruction) reviewed in this study. These indicators play a significant role in Kentucky's school improvement model (McKinney, 2007) yet no formal psychometric analysis has been conducted on any of the nine standards of the SISI document at the high school level. An analysis of the complete set of standards is beyond the scope of this study. 
Eigenvalue and scree plots were calculated and plotted along with principal components analysis for the three sets of indicators considered in this study. A single factor was represented for each Standard. For Standard 7 (Leadership), this factor explained $45.4 \%$ of the variance among the 11 indicators. Cronbach's coefficient alpha was computed on the single factor to determine internal reliability of the scale. A composite alpha of .862 indicated a high degree of internal reliability. Along with the factor analysis, this supports the KDE's intention that the 11 indicators for Standard 7 (Leadership) are consistent for the overall measurement of this variable, indicating a single, strong school leadership construct.

For Standard 1 (Curriculum), the principal components analysis, eigenvalues, and scree plot demonstrated a single factor represented the standard, explaining $52.8 \%$ of the variance among the seven indicators. Cronbach's coefficient alpha was computed on the single factor to determine internal reliability of the scale. A composite alpha of .824 indicated a high degree of internal reliability. The factor analysis and the Cronbach's alpha together provide evidence that the seven indicators for Standard 1 (Curriculum) are consistent for the overall measurement of this variable. This supports KDE's development of Standard 1 as a single construct representing curriculum.

As with Standards 7 (Leadership) and 1 (Curriculum), eigenvalues and a scree plot were determined for Standard 3 (Instruction) to go with the principal components analysis that was conducted for Standard 3 (Curriculum); a single factor represented the standard. A variance of $48.2 \%$ was explained among the eight indicators. Cronbach's coefficient alpha was computed on the single factor. The composite alpha of .818 , indicated high internal reliability. As with the previous two standards, KDE's development of the eight indicators for Standard 3 (Instruction) as a single construct is supported, with excellent psychometric 
properties.

For the three standards, the inter-scale correlations represent one aspect of external criterion validity (Nitro, 2003). All these reflect ideal psychometric properties, i.e., the standards are inter-related, as happens for components of a total school improvement model. Yet, the three standards are somewhat independent of each other. The three intercorrelations range from .239 to .625 . Analysis

The SISI document is the scaffold for improving Kentucky schools. Since its inception, hundreds of schools across the Commonwealth have conducted audits and/or reviews under the conjecture of school improvement. In this study, 83 Kentucky high schools were either audited or reviewed during 2001-2005. The standards and their respective indicators, based on the SISI document, are the assessment and evaluative instrumentation utilized for the Scholastic Audits. Because these audits drive Kentucky school improvement efforts, it is important that these standards and indicators have their psychometric properties verified.

This study provides that verification for high schools for Standard 7 (Leadership), Standard 1 (Curriculum), and Standard 3 (Instruction), confirming the strong psychometric quality of these three standards from the larger set of nine in the SISI document. KDE had conducted preliminary examination of the standards, but only to ascertain whether specific indicators from among the overall set of 88 could distinguish between high and low scoring schools. These were designated as leverage points but were not focused on the separate standards and did not disaggregate elementary, middle, and high school samples (McKinney, 2007; personal communication, D. Smith, November 10, 2010). KDE had contracted for an independent study of the validity of the standards and indicators that 
comprised the Scholastic Audit but this analysis (Koger \& Thacker, 2004) focused on process and further work on indicators that distinguished successful and failing schools (later renamed as variance points).

Regarding formal psychometric analysis, the dissertations guided by Stephen Miller and Douglas Smith at Western Kentucky University constitute the sole empirical work on the standards. Three previous studies (B. C. Ennis, 2007; McKinney, 2007; Saravia, 2008) along with other analyses (Smith, Harvey, Hammock, \& Miller, 2009) have confirmed the construct validity and reliability of all nine Standards from the SISI document as well as their external criterion validity. However, all these analyses were conducted at the elementary level.

The current study represents the only analysis of Kentucky's Standards and Indicators for School Improvement for high schools. KERA has been recognized as the most comprehensive of all state school reform packages (Pankratz \& Petrosko, 2000; Petrosko, 2000; Steffy; 1993). Further, the Scholastic Audit has been noted as among the seminal efforts in the school improvement literature (Mintrop, 2003; Mintrop \& Trujillo, 2005). It is thus incumbent that the underlying instrumentation in that process be valid and reliable. This study provides that evidence for Standards 1,3, and 7 for work with high schools. In that regard, it is worth repeating that this is the sole study of Kentucky's Scholastic Audit database that has been conducted at the high school level. Given Kentucky's prominence in the school reform and accountability movement, this study takes on special significance.

\section{Research Question 1}

To what degree do demographic factors affect Standard 7 (Leadership), Standard 1 (Curriculum), Standard 3 (Instruction), and the Academic Index in Kentucky high schools? 
Four separate simultaneous regressions were conducted to address Research Question 1. The nine independent variables for this research question included Appalachian, County vs. Independent school district, Percent Disability, Percent Free/Reduced Lunch, Percent Gifted, Percent Limited English Proficiency, School Size (grades $10 \& 11$ ), Percent White, and Year of Audit. This study adds to the available information regarding influence of demographic factors on student achievement as indicated by the Academic Index. In addition, it provides a statistical analysis of the relationships between demographic factors and Standard 7 (Leadership), Standard 1 (Curriculum), and Standard 3 (Instruction).

Although the model demonstrates a significant relationship between the independent variables and Leadership, $p<.001$, none of the individual variables considered yield a significant effect. Standard 7 (Leadership) is essentially independent ( $12 \%$ variance) of the demographics of the student population of the high schools for this sample. Standard 1 (Curriculum) is also independent regarding the demographic factors examined as none were significant. However, Standard 3 (Instruction) is affected by the demographic factors of Percent Free/Reduced Lunch and Year of the Audit as both produce a significant effect. Eighteen percent of the variance in Standard 3 (Instruction) is explained by the Demographic Variables with Percent Free/Reduced Lunch having the strongest beta, -.395 indicating a negative impact. Year of the Audit also produced a substantial effect on Standard 3 with a standardized beta of .281 .

The Adjusted $R^{2}$ of .65 indicates a very strong effect of the Demographic Variables, almost two-thirds of the variation in the Academic Index when it is regressed on the demographic factors. Several Demographic Variables produced significant effects: Percent White (beta $=.259)$, Percent Free/Reduced Lunch $($ beta $=-.380)$, Percent Gifted (beta $=$ 
.149 ), County/Independent district (beta $=-.208$ ), and Year of Audit (beta $=.463$ ). Year of Audit and Percent Free/Reduced Lunch have the strongest effects. The negative beta for County/Independent districts is because schools from independent districts (coded 0) had higher achievement in this database of audited high schools.

Analysis

The findings of this study indicate that both Standard 7 (Leadership) and Standard 1 (Curriculum) are, for the most part, independent of influence from the individual demographics of a high school's student population. However, Standard 3 (Instruction) is affected--an 18\% variance--with the greatest impacts from Percent Free/Reduced Lunch and Year of Audit. The findings of this study support other research that low-performing schools suffer from the negative effect on instruction (Darling-Hammond, 2004; Moore, 2003; Roeder, 2000). Substantial research reveals that the best teachers migrate to the more advantaged schools (Ladd \& Walsh, 2002; Roeder, 2001). Beck and Foster (1999) stated that the concept of school is traced back to a time when schools typically supported local values and thus are both reflections and extensions of their communities. DeYoung (1983) indicated that lower-performing schools which have a higher percentage of students on free and reduced lunch (an indicator of poverty) may be in districts that have less local wealth to tax for educational programs, including instructional resources. Year of Audit has a positive effect on Standard 3 (Instruction) indicating that in the era of educational reform, student achievement in Kentucky high schools continues to inch upward year after year.

Student demographics have a very strong effect on the Academic Index. The variables accounting for $65 \%$ of the variance are Percent White, Percent Free/Reduced Lunch, Percent Gifted, County/Independent district, and Year of Audit. Percent White is a 
positive factor on student achievement. Although educators across the state and nation continue to struggle with closing the achievement gap, the findings of this study are consistent with general research (L. S. Miller, 1995; S. K. Miller \& Moore, 2006; Roeder, 2001) that more work is needed for reductions in achievement gaps (the equity dimension) to be evident. Percent Gifted has is a significant effect on the Academic Index; however, a disconcerting realization, especially in light of school accountability of academic progress, is that struggling schools are likely to have fewer students identified as gifted (Moore, 2003).

County/Independent factor has a negative effect on the Academic Index. DeYoung (1983) stated that county school districts in Kentucky have poorer financial characteristics than do independent school districts. The independent districts which lie mostly in manufacturing areas have a higher tax basis, compared to non-manufacturing areas, with which to support educational programs. The county school districts, according to DeYoung, have student populations supporting more students scoring below average on basic skill tests in reading and mathematics. And, the county school districts generally have higher percentages of disadvantaged children and higher pupil/teacher ratios.

The Demographic Factor of Percent Free/Reduced Lunch, an indicator of poverty, is one of the more powerful predictors for the Academic Index. This variable has a welldocumented history of significance in educational research (e.g., Guskey, 1997; Lyons, 2004; Moore, 2003; Roeder, 1999, 2000, 2001). All schools in Kentucky are making academic gains; however, for the schools in this study (the lower one-third of struggling schools), how to overcome the snares of student disadvantage remains a concern. Hallinger and Murphy (1985) suggested that leadership may well need to adjust school practices to fit the disadvantaged environment, that is, what works for individual schools may be 
different in varying socioeconomic levels.

Year of Audit is one of the strongest factors on the Academic Index, an indication of the continued progress toward accountability goals for Kentucky high schools. Overall, schools in this study are making academic gains each year, a testimonial to the reforms inscribed in the KERA initiatives. However, of great concern is whether all Kentucky schools can reach proficiency by 2014; Roeder's (2001) work suggests that not all Kentucky schools are on track to reach this goal.

\section{Research Question 2}

To what degree does Standard 7 (Leadership) affect Standard 1 (Curriculum), Standard 3 (Instruction), and the Academic Index in Kentucky high schools?

Three separate simultaneous regressions were used to address Research Question 2. The first and second regressions determined the effect of Standard 7 (Leadership) on Standard 1 (Curriculum) and Standard 3 (Instruction), respectively. Leadership yields a significant effect on Curriculum, explaining $40 \%$ of the variance in Standard 1, a modest effect. The effect of Standard 7 (Leadership) on Standard 3 (Instruction) is significant with a .39 effect size. The third regression of the Academic Index on Standard 7 (Leadership) is significant as well. The effect size of .20 indicates that one-fifth of the variation in the Academic Index is associated with Standard 7 (Leadership).

Analysis

The first indicator (1.1.a) of the first standard (Curriculum) in the SISI document states "evidence that the curriculum is aligned with the Academic Expectations, Core Content for Assessment, Transformations and the Program of Studies" (KDE, 2004a, p. 32-33), suggesting to schools and school districts alike that the initial step in Kentucky's accountability model is the alignment of curriculum with the accountability goals. The 
findings of this study validate that Standard 7 (Leadership) plays a significant function in directing Curriculum, i.e., $40 \%$ of the variation in this set of indicators, for Indicator 1.1.a and the others that comprise Standard 1, is explained due to Leadership.

Standard 7 (Leadership) also yields a significant effect on Instruction, explaining $39 \%$ of the variance on the Standard 3 factor that underlies the seven Instruction indicators. The weight of this influence provides an indirect avenue for principals to affect accountability scores. Roeder (2000) suggests that not only does good instruction coupled with rigorous curriculum improve student academic success but may also be an important factor with respect to positive results for children of disadvantaged socioeconomics. In the SISI document, Standard 3 (Instruction) Indicator 3.1.c states "instructional strategies/activities are consistently monitored and aligned with the changing needs of a diverse student population to ensure various learning approaches and learning styles are addressed" (KDE, 2004a, p.34-35).

Instruction is perhaps the most significant means through which principals in Kentucky high schools can indirectly influence accountability scores. As Hallinger et al. (1990) note, principals influence student learning by developing an instructional focus for teachers throughout the school that shapes the school's instructional climate and instructional organization. This study provides empirical evidence that Standard 7 (Leadership) does have a direct and substantial effect on the quality of Instruction (Standard 3) in Kentucky high schools, based on the Scholastic Audit data. This is significant for several reasons. First, it extends McKinney's (2007) work on elementary schools; here the results are very similar for high schools. Second, there is a dearth of empirical analyses that examine the effect of principal's leadership on curriculum and instruction for high schools. This study addresses that issue directly. Finally, there is the 
prominence of Kentucky in the state school reform and accountability movements. That this study utilizes Kentucky data adds to its importance in the field.

\section{Research Question 3}

To what degree do Standard 1 (Curriculum) and Standard 3 (Instruction) affect the Academic Index in Kentucky high schools?

Simultaneous regression was used to analyze the effect of Curriculum and Instruction on the Academic Index. Results indicate that the model is significant, explaining $39 \%$ of the variance in the Academic Index, the dependent variable. However, the entire effect of the model comes from Standard 3 (Instruction) as Standard 1 (Curriculum) has no impact on the Academic Index. Instruction has a positive effect of .647 ; a one unit increase in Instruction would generate a 6.47 unit gain in the Academic Index.

Analysis

This study reiterates the importance of Kentucky's initiatives surrounding instructional leadership to advance school improvement and thus student success. The findings confirm the overwhelming consensus in current literature that quality instruction influences student achievement (Darling-Hammond, 2004; Ingersoll, 2003; Schlechty, 2001). The literature on Instruction indicates that the cultural and pedagogical strategies employed in the classroom affect student achievement. In planning lessons, teachers are influenced by many factors that determine what content they teach, how they teach it, and what materials they use to engage students (Weiss et al., 2003). Tying this result back to Research Question 2, these contextual influences on quality instruction must be addressed by the instructional leadership for increased student achievement to occur (Weiss \& Pasley, 2004). That injunction is consistent with the SISI document (Standard 3, Indicator 
7.1.a) which directs school leadership to "develop and sustain a shared vision" (KDE, 2004a, pp. 34-35). Finally, this study also extends the work of McKinney (2007) on the Scholastic Audit database from elementary to high schools. The results here mirror McKinney's almost exactly. Likewise, this study increases the extant work on the effects of curriculum and instruction at the secondary level, where very little empirical work has been conducted compared to elementary level investigations.

\section{Research Question 4}

To what degree do Standard 1 (Curriculum) and Standard 3 (Instruction) mediate the effect of Standard 7 (Leadership) on the Academic Index, controlling for demographics in Kentucky high schools?

Hierarchical multiple regression was used to address Research Question 4, incorporating all the independent variables to establish the effects of Demographic Factors, Standard 7 (Leadership), and Standards 1 (Curriculum) and 3 (Instruction) on the Academic Index. All Demographic Factors were included. Each of the three steps of the model was significant. Calculating the residual sums of squares of the models, which are separate calculations, produced the full and reduced model $F$ test. The calculations indicated whether the model was improved by adding the new variables, i.e., going from Step 1 (Demographics only) to Step 2 (Leadership was added) then on to Step 3 (Curriculum and Instruction were added).

For Step 1, the Demographic Factors (Adjusted $R^{2}=.65$ ) indicate a large affect on the Academic Index. The factors--County/Independent district $($ beta $=-.208)$, Percent Gifted $($ beta $=.149)$, Percent Free/Reduced Lunch $($ beta $=-.380)$, Percent White (beta $=$ .259 ), and Year of Audit (beta $=.463$ )--are all statistically significant at the $p<.05$ level. Controlling for demographics, Standard 7 (Leadership) was added to the equation in Step 2 that produced a change in the Adjusted $R^{2}$ of .06; Standard 7 was significant with a 
standardized beta $=.284$. Percent Gifted was no longer significant. In Step 3, to determine the extent that Standard 7 (Leadership) mediates the Academic Index, Standards 1 and 3 (Curriculum and Instruction, respectively) were added, with Demographic Factors from Step 1 controlled. An additional incremental change of .05 in the Adjusted $R^{2}$ was produced. In this final step, Standard 1 (Curriculum) was not significant; however, Standard 3 (Instruction) was significant. All other significant variables from Step 2 remained significant for Step 3 . The total variance of .76 in the final model is an unusually high effect in educational research.

From Step 2 to Step 3, Standard 7 (Leadership, beta $=.215$ ) and Standard 3 (Instruction, beta $=.279$ ) were significant, controlling the Demographic Factors. For Step 3, the final hierarchical model, Year of Audit (beta $=.373$ ), Percent White (beta $=.318$ ), Percent Free/Reduced Lunch (beta $=-.226$ ), and County/Independent district (beta $=-.194$ ) all remained significant. Worth mentioning is the fact that the beta score for Instruction (.279) exceeds that of Percent Free/Reduced Lunch (-.226), an indicator of poverty, while the direct influence of Leadership (beta $=.215$ ) is almost as high validating that the practices of school leadership, from classroom teachers to school level leadership to district leadership, can have a significant impact, even when demographics seem insurmountable. This final regression confirms the mediated effects model of leadership, with influence through instruction but not curriculum.

Analysis

In these high schools, the Percent White is a factor in school academic success and student achievement. This extends the work of McKinney (2007) who states "the Percent White influence continues to demonstrate a discernible gap in achievement compared to minorities" (p. 155). Beck and Shoffstall (2005) reported that data analysis of the State of 
Illinois achievement tests showed how strongly associated the socioeconomic characteristics of schools are with school level outcomes. "Factors beyond individual school control, such as the percentage of students who are poor, the percentage who are black, and the residential mobility of students' families explain up to $80 \%$ of the variance in school-level outcomes" (p. 1).

The County vs. Independent demographic poses a weak effect (beta $=-.208)$ on the Academic Index. The sample for this study is 83 Kentucky high schools-- $80 \%$ County districts, 20\% Independent districts. Although the findings reveal Independent schools fared higher on the Academic Index than County schools, the reason schools participated in the audits, mandatory versus voluntary, was not made available in this data set. An additional consideration is that most of the schools comprising this database are considered low-performing schools, i.e., required to participate in the Commonwealth's school improvement process, based on Scholastic Audit diagnostic guidance. Thus, the sample may not be indicative of all school districts in the state accountability system, the majority of which were not forced into this remedial effort.

For this study, Year of Audit (beta $=.46$ ) is the most influential factor in school improvement, an indicator that the Kentucky schools in this study continue to make progress year after year. As S. K. Miller and Moore (2006) noted, the overall progress under KERA (the excellence dimension) is substantial and sustained. However, the influence of demographic factors (the equity dimension) has not been eliminated or reduced. Roeder's (2001) study raised the question of whether expecting all Kentucky schools to reach the goal of proficiency by 2014 is realistic.

As expected, the demographical factors of a school's student population play a pivotal role in a school's overall academic success, confirming the results of the McKinney 
(2007) study as well as others. In particular, the Percent Free/Reduced Lunch students have one of the more augmented impacts on the Academic Index second only to Year of Audit. A one unit increase in this variable creates a decrease of 22 units in the Academic Index. This presents an intimidating obstacle to consider as principals and others work toward meeting the goal of proficiency by 2014 . Although Kentucky provides additional financial support to schools toward assisting their disadvantaged students, the state does not make adjustments when determining the Accountability Index. These criteria may play a role in determining the outcome of how many schools meet the proficiency goal.

The schools that began with low baseline scores which are typically the schools with high numbers of socioeconomically disadvantaged students must achieve much higher gradients of improvement in achievement levels than the more affluent, higher achieving schools with greater resources. This raises a separate issue for the accountability movement, one which is seldom acknowledged explicitly (cf. S. K. Miller \& Moore, 2006). It is one thing to expect all schools to improve their quality and their overall achievement levels. It is quite another to require that lower achieving (typically with higher proportions of at-risk students) to catch up with higher achieving schools (typically with more affluent pupils). Not only does this impose the burden of a faster rate of improvement for the schools that start out behind, but it also suggests that affluent parents would sit idly by as these lower achieving schools got better faster than the schools which their more privileged children attend. As one of the pioneers in the school effectiveness literature (Edmonds, 1979) remarked long ago that is not likely to occur. Those politically savvy, affluent parents would demand that the practices that were working better for disadvantaged schools be appropriated for their own children. Closing achievement gaps does not happen in political vacuum. 
Despite these political realities, this study does have positive news regarding school improvement. Two significant independent variables are central to this study: Leadership and Instruction. These two variables accounted for an additional $6 \%$ and $5 \%$, respectively, variance on the Academic Index, controlling for the Demographic Factors. The effects are not large in comparison to variables such as Year of Audit and Percent Free/Reduced Lunch; however, they are certainly striking as they are alterable processes that schools can manipulate (Bloom, 1980). Additionally, it is worth mentioning that Leadership enters in Step 2 and Instruction in Step 3 of the hierarchical regression, thus providing strong empirical support for the mediated effects model of instructional Leadership (cf. Hallinger \& Heck, 1998; Murphy, 2004) and the theoretical framework for this study.

In addition, the current findings complement the work of Owings et al, (2005). Their statewide study of Virginia principals found a significant relationship exists between principals' quality (measured by the ISLLC Standards) and student achievement (measured on the Virginia Standards of Learning tests); schools with the higher rated principals had higher student achievement, controlling for socioeconomic status.

\section{Recommendations}

Twenty years have passed since Commonwealth legislators signed into law the Kentucky Education Reform Act (KERA)--only four years remain until all schools in Kentucky must attain Proficiency (an Accountability score of 100 out of a possible 140). Some of Kentucky's schools have met the Proficiency goal; others are making adequate progress toward the goal. Yet, for many schools the idea that 2014 is only four years away is a threatening admonition as the possibility of meeting the goal of proficiency looks ever more unlikely (Roeder, 2001). Using data for stable schools, Lindsey (2007), projected that by 2014 only $37 \%$ of Kentucky schools will be proficient, $50 \%$ will be in the progressing 
category, and $13 \%$ will be in the assistance group.

Although standards and high-stakes accountability alone have been insufficient for achieving goals for all schools and all students within the state, the effects of Leadership, Curriculum, and Instruction noted in this study offer optimism for increased academic success. In the subsequent paragraphs, implications to policy and practice are discussed. In addition, suggestions for future research are offered.

\section{Implications for Policy}

First, in this study Year of Audit significantly affected on the Academic Index indicating that Kentucky schools are climbing the upward slope toward Proficiency. Since the SISI document is the primary scaffold for school improvement, particularly for underachieving schools, the results of this study as well as the findings from B. C. Ennis (2007), McKinney (2007), and Saravia (2008) indicate that the SISI functions as intended for whole school improvement. Therefore, rather than the Scholastic Audits and Reviews be used as punitive for struggling schools--which Mintrop (2003) reported could serve to reduce teacher motivation--the audits/reviews should be promoted by KDE policy for all schools (County and Independent; successful, meeting, and low-performing). Since successful schools are not required to conduct the Scholastic Audits, many have opted out of this process. However, policies including successful schools in the audits could be a useful practice as it would amplify the understanding of accountability over the entire scope of improvement--from the lowest-performing schools to those achieving the accountability goals.

Second, the framers of KERA wisely integrated the concept of the instructional leader in the reform initiative, including the ISLLC standards and the SISI document. Additionally, the Effective Instructional Leadership Act firmly recognized the principal as 
responsible for building level instructional leadership. The findings of this study support the positive direct and indirect effects of school level leadership on the Academic Index. However, as McKinney (2007) stated “...little provision was made for the cultural indoctrination needed to embed the concept (of instructional leadership) in practice....Extensive training/professional development will be necessary to root out deeply embedded but ineffective models of instruction and leadership" (p. 168). Therefore, to complement the guidelines and standards for instructional leadership practices in place, Kentucky should embellish principal preparedness programs to develop leaders of a worldclass educational system.

Third, as Guskey (2003) noted, instruction matters. He cited examples of success in disadvantaged schools where some teachers had students making remarkable gains while other teachers with the same students had only moderate success. Roeder (2001) also suggested possible improvements in school performance for lower-performing schools lies with teaching--good teaching has an especially large and positive impact for disadvantaged children. In addition, Ladd and Walsh (2002) and Roeder (2001) suggested that schools with higher-ability students may in fact be more effective than schools with lowperforming students because "they may attract higher quality teachers who can use their seniority to move to schools where the students are more motivated and easier to teach" (p. 5). Should Kentucky draft policies that create statewide standards, similar to the National Board for Professional Teaching Standards (NBPTS) to further promote effective classroom instruction? Further, if Kentucky is to address fully a successful response to disadvantaged schools and instruction quality, should the state offer incentives to attract the best teachers to low-performing schools?

Finally, Roeder (2001) offered that the first and most obvious tool to deal with 
inequalities across Kentucky's school systems would be more equitable funding across school districts (the motivation for the original suit that resulted in KERA). Some argue that poorer schools with more social disadvantages do not need equal resources; they need more resources. If all schools and all students are to reach Proficiency, the proverbial bar for academic success must be raised in this group of students. Serious attention to the needs of these students will require momentous adjustments in resource allocations in order for all children to embrace equal opportunities for success.

\section{Practice}

Several findings from this study suggest consideration. First, substantiated results of previous studies (B. C. Ennis, 2007; McKinney, 2007; Saravia, 2008) and the current study are consistent that standards and high-stakes accountability have a positive influence on Kentucky schools. More specifically, the Standards and Indicators for School Improvement (SISI) document--an instrument of whole school reform--have Kentucky schools climbing the upward continuum toward Proficiency, as indicated by the Year of Audit effect on the Academic Index. Second, Standard 3 (Instruction) does have an impact on the Academic Index, a finding consistent with the literature on instruction generally (Darling-Hammond, 2004; Ingersoll, 2003; Schlechty, 2001). Third, Standard 1 (Curriculum) does not have a significant effect on the Academic Index. These trends are addressed below.

First, could a contributing factor to the lack of effect for Standard 1 (Curriculum) be that the state's learning content is driven by a set of established guidelines: the Program of Studies, Academic Expectations, and Core Content? Because of these documents, the Curriculum guesswork is removed--these directives specifically inculcate the Kentucky Department of Education's roadmap for what students should know. Does this suggest 
little variation in how schools encompass curriculum? Or, does it imply that because it is required, schools spend less time considering how they will implement it. Whatever the cause, clearly, more work on "best practices" in curriculum is suggested.

Second, how students should learn remains un-prescribed. According to the findings of this study for Kentucky high schools, Standard 3 (Instruction) alone has an effect of $39 \%$ on the Academic Index--an effect greater than the Percent Free/Reduced Lunch demographic factor, an indicator of social disadvantage for students. Yet, even considering the Commonwealth's passage of the most comprehensive education reform ever legislated by a state (Steffy, 1993), the practice of instruction has no definitive roadmap. Whole school reform has the SISI document; Curriculum has the Program of Studies, the Academic Expectations, and the Core Content; and instructional leadership has the ISSLC standards.

Kentucky schools as well as those across the nation grapple to find ways to improve instruction as it has a direct effect on school and student success. Lake et al. (1999) found that schools making a significant gain in test scores took a pro-active approach toward assessing strengths and weaknesses, and focusing on improving instruction. Cowley and Meehan (2003) suggested that for schools to address successful academic achievement, not only should faculty focus on accountability requirements, but also on school climate to sustain teacher commitment to continual improvement. Thus, the findings of Weiss and Pasley (2004) are sobering: nine out of 10 times $(N=364$ math and science lessons across the nation), teachers have a sense of autonomy in choosing how to implement lessons; teachers have little external guidance in designing their instruction; teachers use resources and strategies based on their knowledge, beliefs, and experience. Weiss et al. concluded $15 \%$ of K-12 lessons are considered high in quality, $27 \%$ medium 
in quality, and $59 \%$ low in quality.

Laying blame for less than quality instruction and/or the lack of student achievement in the laps of teachers is not the intent, quite the contrary. Rather, this recommendation addresses the potential to minimize the guesswork from the practice of Instruction: the creation and implementation of a statewide Instructional Roadmap, that is, a uniform state instructional framework (content-specific timelines, instructional strategies, and assessments) coinciding with Standard 1 (Curriculum) documents: the Program of Studies, Academic Expectations, and Core Content.

Consider what this could mean to a new or inexperienced teacher (and school effectiveness) to be handed an Instructional Toolkit (framework) that (a) includes Best Practice-based instructional lessons aligned with the Program of Studies, Academic Expectations, and Core Content; (b) eliminates guesswork on what is effective instruction; (c) affords feedback for student remediation from content-specific departmentalized assessments; (d) provides resources for prescribed activities; (e) addresses differentiated instruction for diverse classroom populations; and (f) outlines the pace of instruction for the school year. Consider what it could mean to school leadership to have a uniform state Instructional Toolkit for all content areas within the school building. Consider how this could improve student achievement.

Third, in Kentucky, the Accountability Index derived from CATS scores and nonacademic factors determines if schools are succeeding or not. Based on the results, schools are mandated or volunteer for a Scholastic Audit in which the SISI document is used as the assessment and evaluative instrument. The audits that are conducted by KDE-trained teams provide the guidelines for school improvement. At least every two years, all Kentucky schools should complete a mandatory Scholastic Audit. 
Fourth, realizing the implications of ill-prepared school leadership, in April 2005 Kentucky began looking at the practicality of preparation programs. Taking recommendations for redesigning principal preparation from a Leading Change conference, two groups [Kentucky's State Action Education Leadership Project (SAELP) and Jefferson County's Leading Education Achievement in Districts (LEAD) Project] merged, forming the Kentucky Cohesive Leadership System (KyCLS). In the fall of 2006, House Joint Resolution 14 (HJR 14) provided legislative support to move the work forward. Funded by Kentucky's Wallace Foundation, KyCLS facilitated discussions with stakeholder groups including local, state, and national leadership. In May 2008, the KyCLS presented the Continuum for Principal Preparation and Development document. Heralded as a driving force in developing world-class leadership in Kentucky, the Continuum has the potential to give aspiring principal candidates the knowledge, skills, and behaviors to become highly effective instructional leaders from a cutting-edge, research-based approach (KyCLS, 2008). The crucial point becomes how much are Kentucky legislators and KDE willing to support this initiative? Will funds continue once the Wallace grant expires? Will Kentucky maintain policies and practices to provide all Kentucky schools, even the disadvantaged ones, with world-class leadership?

Finally, because the reality of socioeconomic disadvantage so greatly affects Kentucky students' academic success and ultimately the future potential prosperity of the Commonwealth, politicians, educators, and private citizens must make it a responsibility to engage in the work of education. The suggestions above, for both policy and practice, are not likely to reach fruition without the support of citizens and interest groups across the state.

Future Research 
The period for the current study hits the midpoint of the Kentucky Reform Era. KERA was enacted in 1990, the study period is 2001-2005, and Proficiency for all Kentucky schools is targeted for 2014. The investigation has added value as it may represent the final study period for CATS as Kentucky begins its transition to a new accountability system, effective 2011 (B. Haselton, personal communication, September 24,2010 ). Senate Bill 1, amending KRS 158.6451 , was passed by the 2009 General Assembly. The new bill dismantled Kentucky's Commonwealth Accountability Testing System (CATS) and suspended accountability until the 2011-12 school year. The bill signed into law by Kentucky Governor Steve Beshear on March 29, 2009, directed the Kentucky Department of Education to develop a new system of assessment and accountability. Although the results from CATS are still valid and reliable and may be used to highlight progress (KDE, 2010), work is underway on the new system. This changing political context suggests several directions for future research.

First, as the state embarks on this new layer of Kentucky educational reform, research is certainly warranted on the execution and attainment of the accountability goals. Regardless of new goals or new assessments, children's learning depends on what happens as they interact with teachers while engaging the curriculum (Lockwood, 1994).

Second, this study examines only a part of the KDE secondary database, that is, the Scholastic Audits from 2001-2005. The sample of students in this study entered school before the implementation of programs such as Ungraded Primary. Future research should include Scholastic Audit data beyond 2005.

Third, the SISI document contains nine standards, all of which are intended for use in whole-school improvement decisions. To date, there are three completed studies that examined the SISI standards: B C. Ennis (2007) examined Leadership and Professional 
Growth, Development and Evaluation; McKinney (2007) examined Leadership, Curriculum, and Instruction; and Saravia (2008) examined School Culture and Parent Involvement. The target samples for the three studies were Kentucky elementary schools. This study examined Leadership, Curriculum, and Instruction at the high school level. Six of the nine standards have been examined; the opportunities for further research that spring from this work are numerous, from other combinations, to those not yet examined, to the overall SISI data set.

Fourth, the current research only included Kentucky high schools, grades 9-12. The three completed studies mentioned above considered only elementary schools, grades 4 and 5. It is also important to mention that most of the schools in the study were struggling, thus the Scholastic Audit. Some schools in the study volunteered for the Scholastic Review and therefore were not considered as an at-risk school. Future research should be extended to include all grade levels as well as all Kentucky schools.

Fifth, data for this study and the previously mentioned studies of B. C. Ennis (2007), McKinney (2007), and Saravia (2008) were used from schools that went through the Scholastic Audit process. The KDE secondary database does not provide longitudinal evidence that the schools conducting audits actually improved their Accountability Index in the successive testing cycles. Such longitudinal studies could surely be of tremendous benefit toward deciphering goals for school improvement.

Sixth, is there a way to level the effects of demographics for students of disadvantage on the Academic Index? Kentucky initiatives for socially-disadvantaged students currently number 22 . That is, there are 22 programs specifically directed at minimizing socioeconomic distress for students and their families. Yet, as this study and others have shown, the effects of disadvantage continue to play havoc for both student 
level academic and school level success, based on high numbers of Percent Free/Reduced Lunch students, an indicator of poverty. For the sake of the accountability movement but more importantly for the students who have not benefited from reform initiatives, equitable solutions for the socially disadvantaged students are critical. This is an area of opportunity for researchers--finding alternatives for children who have been traditionally neglected or unable to profit from existing programs.

Last, five percent of teachers in the Commonwealth of Kentucky have earned National Board Certification. Do these teachers' students achieve greater academic success? If so, what are the implications for university teacher preparation programs versus mandated national board certification? What are the implications for student achievement?

\section{Conclusions}

In 1988, the Commonwealth of Kentucky found itself the defendant in a lawsuit pitted against school districts within its own state boundaries. As a result, in 1989, the Kentucky Supreme Court ruled the state educational system unconstitutional, paving the way for Kentucky to be thrust into the national spotlight as state legislators and the Kentucky Department of Education (KDE) created the most comprehensive educational package ever passed by a legislative body (Steffy, 1993), the 1990 Kentucky Educational Reform Act (KERA). Since its inception, KERA has been heralded and condemned. It has been researched and tweaked. Even now, 20 years later, KERA continues to evolve as it sustains the pressures of time.

The framers understood the need for guidelines and goals if the educational reformation was to create a successful school environment for all the state's children. To provide the guidance, strategies, and vision for this objective, the KDE developed a model extended to whole school reform, Kentucky's Standards and Indicators for School 
Improvement (SISI); the goal established for all Kentucky schools is to achieve Proficiency (a composite score of 100 out of 140 calculated from CATS scores, the Academic Index, and non-academic factors) by the year 2014. Nine academic and leadership standards plus 88 indicators meter the process while the Scholastic Audit provides an evaluative tool for schools to assess their progress toward the Proficiency goal. The Scholastic Audit, KDE's effort to understand the needs of struggling schools, provides a unique and recorded glimpse into how standards and accountability influence classroom learning (McKinney, 2007) as well as the daily life of the school itself. The Scholastic Audit data was compiled by $\mathrm{KDE}$ trained teams who rated the schools by assigning behaviorally defined score values (1-4) to the indicators for each of the nine standards. By using the compilation of the KDE secondary database for the years 2001-2005, this study provides evidence of the functionality at the high school level of three of the nine standards included in the model: Standard 7 (Leadership), Standard 1 (Curriculum), and Standard 3 (Instruction).

The purpose of this study was to: (a) examine the effects of school level leadership on curriculum and instruction as they pertain to student success depicted by the Academic Index scores in Kentucky high schools, and (b) to investigate relationships, if any, that may exist among certain demographic factors such as school size, school setting, gender, and student achievement. Previous studies (B. C. Ennis, 2007; McKinney, 2007, and Saravia, 2008) have examined standards within the SISI document; all three were at the elementary level. No previous studies have examined the standards from the SISI document at the high school level.

This study provides the following results: (a) Demographic Factors have a significant and most compelling effect on the Academic Index (effect size of .65); (b) Instruction has a significant effect on the Academic Index; (c) Curriculum has no effect on 
the Academic Index, (d) Leadership has a significant direct effect on the Academic Index; (e) Leadership also has a significant indirect (mediated) effect on the Academic Index through Instruction.

This study indicates that Standard 7 (Leadership) yields a significant effect on Curriculum, explaining a 40\% variance, and on Standard 3 (Instruction), a 39\% variance. The leadership effect is essential considering this study found the strongest correlations measured to be huddled around the standards of this study--Standard 7 (Leadership), Standard 1 (Curriculum), and Standard 3 (Instruction) as they relate to each other and the dependent variable, the Academic Index. The highest correlation was between Standard 7 (Leadership) and Standard 3 (Instruction), $r=.629$, which becomes a substantial corridor for the principal to have a mediated effect on academic success. In the hierarchical multiple regression, when Curriculum and Instruction were added to consider the extent that Standard 7 (Leadership) mediates academic performance with Demographic Factors controlled, Instruction was significant, while Curriculum added nothing. Thus the final regression confirmed the mediated effects model of Leadership (Murphy, 2004), with influence through Instruction but not Curriculum.

Standard 7 (Leadership) also has a significant direct impact on the Academic Index, an effect size of .20. Taken together, these results support the accord that principals do make a difference in student achievement. Beyond Murphy (2004) and others that the influence of the principal is most often mediated through others, that is, an indirect influence as opposed to direct influence, this study demonstrates both a direct and indirect effect. The 11 indicators of Standard 7 (Leadership) provide a guideline as to how effective principals provide instructional leadership. And, although it is beyond the scope of this study to attempt interpretation of how principals "do" leadership, Bossert et al. (1982) 
expressed the principal's effect on Instruction as creating the condition for successful schooling by providing coherence to their schools' instructional programs and conceptualizing instructional goals.

Because the Scholastic Audit is legislated for struggling schools, the student population representing the audited schools is generally more disadvantaged (higher Percent Free/Reduced Lunch) compared with the average Kentucky high school. Schools in Kentucky must work hard to achieve their goals; however, policymakers also need to consider what is evident in education (and supported by the results of this study): the impact of demographic factors on the Academic Index, more specifically Percent Free/Reduced Lunch, an indicator of poverty. In his findings, McKinney (2007) said:

It cannot be over emphasized that in this study, despite the contributions of Leadership and Instruction to the Academic Index, and the overall confirmation of both the Scholastic Audit and the mediated effects of leadership, the overwhelming influence on achievement $(60 \%)$ was the demographic factors. This represents an unlevel playing field that cannot be ignored and confirms the findings of a number of other major studies of Kentucky's accountability. (p. 175, emphasis in the original)

McKinney's (2007) findings are relevant for this analysis as the same database and methodology were used, only for elementary schools. His results are similar to those of this study; however, in this study of audited Kentucky's high schools during the period of $2001-2005$, a variance of $65 \%$ on the Academic Index is explained by socioeconomic factors. As McKinney emphasized, the impact of the Standards is important, but the disadvantage characterized by socioeconomic factors is the most compelling challenge schools are facing to fulfill their responsibilities. That demographic factors have a more pronounced effect on high schools is consistent with Moore's (2003) study of accountability in Jefferson County Public Schools, by far the largest and most urban district in Kentucky. Looking at seven socio-demographic factors, the Adjusted $R^{2}$ for 
middle and high schools was much stronger than elementary level, reaching $90 \%$ variance explained on three different school-level outcomes--mathematics and reading for CTBS, mathematics and reading for the Kentucky Core Content Tests, and the overall Accountability Index. That finding approaches demography as destiny (cf. S. K. Miller \& Moore, 2006).

The demographic composition of a school's student population is certainly a persuasive argument against school improvement. More likely than not, the effects of disadvantage cannot be totally disarmed. Despite this, the current study's findings support the direct and indirect influence of Leadership. The beta score for Instruction exceeded that of Percent Free/Reduced Lunch as it pertains to the Academic Index while the direct influence of Leadership is almost as high--validating that the policies and practices of school leadership, including instruction, have a significant impact even in the face of disadvantaged demographics. The strong empirical support for the theoretical framework of this study, the mediated effects model of instructional Leadership, offers optimism for increased student success. Because these school level processes are alterable, "schools can make a difference" (Brookover, Beady, Flood, Schwetzer, \& Wisenbaker, 1979). There is hope. 


\section{REFERENCES}

Achilles, C. M., \& Price, W. J. (2001). What is missing in the current debate about education administration (EDAD) standards! AASA Professor, 24(2), 8-13.

Alan, R. (2004, March 7). Kentucky school audits offer academic maps. Education Week, pp. 6-7.

Anthes, K. (2002). School and district leadership (No Child Left Behind Brief). Denver, CO: Education Commission of the States.

Beck, F. D., \& Shoffstall, G. W. (2005). How do rural schools fare under a high stakes testing regime? Journal of Research in Rural Education, 20(14), 1-12.

Bennis, W. G. (1989). On becoming a leader. Reading, MA: Addison-Wesley Publishing Company.

Bjork, L. G. (1993). Effective schools-effective superintendents: The emerging instructional leadership role. Journal of School Leadership, 3, 246-259.

Blackmore, J. (1989). Changing from within: Feminist educators and administrative leadership. Peabody Journal of Education. 19-40.

Bloom, B. S. (1980). The new direction in educational research: Alterable variables. Phi Delta Kappan, 61, 382-385.

Bossert, S., Dwyer, D, Rowan, B., \& Lee, G. (1982, summer). The instructional management role of the principal. Educational Administration Quarterly, 18(3), 3463.

Brookover, W., Beady, C., Flood, P., Schwetzer, J., \& Wisenbaker, J. (1979). School social systems and student achievement: Schools can make a difference. New York: Praeger Publishers.

Caudill, H. M. (1963). Night comes to the Cumberlands: A biography of a depressed area. Boston: Little, Brown.

Chance, P. L., \& Anderson, R. B. (2003). The principal's role in standards-based reform: Linking accountability to instructional improvement. Paper presented at the Annual Meeting of the American Educational Research Association, Chicago, IL. 
Commonwealth Institute for School Leaders. (2006). A collaborative leadership pathway to principalship. Retrieved May 26, 2006, from http://www.education.ky.gov

Cook, T. D., \& Campbell, D. T. (1979). Quazi-experiment: Design and analysis issues for field settings. Boston: Houghton Miffen.

Costello, A. B. \& Osborne, J. W. (2005). Best practices in exploratory factor analysis: Four recommendations for getting the most from your analysis. Practical Assessment, Research \& Evaluation, 10(7), 1-9.

Council of Chief State School Officers. (1996). Interstate School Leaders Licensure Consortium: Standards for school leaders. Washington, DC: Author.

Council of Chief State School Officers. (2008). Educational Leadership Policy Standards: ISSLC 2008 as Adopted by the National Policy Board for Educational Administrators. Washington, DC: Author.

Council on School Performance Standards. (1991). Kentucky's learning goals and valued outcomes (Report Summary). Frankfort: State Printing Office.

Covey, S. R. (1992). Principle centered leadership. New York: Fireside.

Cremin, L. A. (1961). The transformation of the school: Progressivism in American education, 1876-1957. New York: Knopf.

Cuban, L. (1984). How teachers taught: Constancy and change in American classrooms, 1890-1980. New York: Longman.

Cuban, L. (1990). Reforming again, again, and again. Educational Researcher, 19(1), 3-13.

Daggett, W. R. (2005). Achieving academic excellence through rigor and relevance. Rexford, NY: International Center for Leadership in Education.

David, J. L. (1992). School-based decision making: Observations on progress. Lexington, KY: The Pritchard Committee for Academic Excellence.

Darling-Hammond, L. (2004). Inequality and the right to learn: Access to qualified teachers in California's public schools. Teachers College Record, 106, 1936-1966.

DeYoung, A. J. (1991). Rural education: Issues and practice. New York: Garland Pub.

DeYoung, A. J. (1995). The life and death of a rural American high school: Farewell, little Kanawha. New York: Garland Pub.

Division of School Improvement, Kentucky Department of Education. (2003). The Scholastic Audit: A report on school improvement in Kentucky. Frankfort: Office of Leadership and School Improvement. 
Dorn, S. M., O'Rourke, C. L., \& Papa-Lewis, R. (1989). Women in educational administration: Nine case studies. National FORUM of Educational Administration and Supervision Journal, 17(4), 23-28. Retrieved November 15, 2000, from www.nationalforum.com/DORNeas.html

Dwyer, D. (1986). Understanding the principal's contribution to instruction. Peabody Journal of Education, 63(1), 3-18.

Dwyer, D., Lee, G., Rowan, B., \& Bossert, S. (1983). Five principals in action: Perspectives on instructional management. San Francisco: Far West Laboratory for Educational Research and Development.

Edmomds, R. (1979). Effective schools for the urban poor. Educational Leadership, 37(1), 15.

Elmore, R. F., \& Fuhrman, S. H. (2001). Holding schools accountable: Is it working? Phi Delta Kappan, 63, 67-72.

English, F. (2000). Psst! What does one call a set of non-empirical beliefs required to be accepted on faith and enforced by authority? [Answer: A religion, aka the ISLLC Standards]. International Journal of Leadership in Education, 3(2), 159-167.

Ennis, B. C. (2007). Professional development, leadership, and accountability scores: Evidence from Kentucky's Scholastic Audits. Unpublished doctoral dissertation, University of Louisville.

Ennis, L. S. (2002). Effects of instructional strategies on seventh grade science achievement as perceived by Kentucky students. Unpublished doctoral dissertation, University of Louisville.

Fennell, H. (2005). Living leadership in an era of change. Journal of Leadership in Education, 8, 145-165.

Foster, J. D. (1999). Redesigning public education: The Kentucky experience. Lexington, KY: Diversified Services Inc.

Gay, L. R. (1996). Educational research: Competencies for analysis and application (5th ed.). Upper Saddle River, NJ: Prentice-Hall.

Guskey, T. R. (1997, March). The relationship between socioeconomic characteristics and school-level performance assessment results. Paper presented at the American Educational Research Association Annual Meeting, Chicago.

Hale, E. L. \& Moorman, H. N. (2003). Preparing school principals: A national perspective on policy and program innovations. Institute for Educational Leadership, Washington, D.C. and Illinois Education Research Council, Edwardsville, IL. 
Hallinger, P., Bickman, L., \& Davis, K. (1990). What makes a difference? School context, principal leadership, and student achievement (NCEL Occasional Paper No. 3). Nashville: National Center for Educational Leadership. (ERIC Document Reproduction Service No. ED 332 341)

Hallinger, P., \& Heck, R. H. (1998). Exploring the principal's contribution to school effectiveness: 1980-1995. School Effectiveness \& School Improvement, 9, 157-191.

Hallinger, P., \& Murphy, J. (1985). Assessing the instructional management behavior of principals. The Elementary School Journal, 86, 217-247.

Hallinger, P., \& Murphy, J. M. (1985). What's effective for whom? School context and student achievement. Planning \& Changing, 16, 152-160.

Hallinger, P., \& Murphy, J. (1986). Instructional leadership in effective schools. The Journal of Educational Administration, 24(2), 213-236.

Hallinger, P. \& Murphy, J. (1995). The superintendent's role in promoting instructional leadership. Administrator's Notebook, 30(6), 1-4.

Hammersley-Fletcher, L. \& Brundrett, M. (2005). Leaders on leadership: The impressions of primary school head teachers and subject leaders. School Leadership and Management, 25(1), 59-75.

Harwell, M., \& LeBeau, B. (2010). Student eligibility for a free lunch as an SES measure in education research. Educational Researcher, 39, 120-131.

Haselton, W. B. (2004). School finance reform in Kentucky: Impact on student outcomes. Unpublished doctoral dissertation. University of Louisville.

Howard, W. (2002). Leadership: Four styles. Education, 126, 384-391.

Ingersoll, R. M. (2003, January 7). To close the gap, quality counts. Education Week, pp. 718.

Institute for Educational Leadership. (2000, October). Leadership for student learning: Reinventing the principalship (School Leadership for the 21st Century Initiative: A Report of the Task Force on the Principalship). Washington, DC: Author.

Jacobs, R. L. \& McClelland, D.C. (1994). Moving up the corporate ladder: A longitudinal study of the leadership motive pattern and managerial success in women and men. Consulting Psychology Journal, 46, 32-41.

Kannapel, P. J. \& Clements, S. K. (2005). Inside the black box of high-performing highpoverty schools. Lexington: Pritchard Committee for Academic Excellence.

Kentucky Cohensive Leadership System (2008). Continuum for Principal Preparation and 
Development. Frankfort: Author.

Kentucky Department of Education. (1993). Transformations: Volumes $1 \& 2$. Frankfort: Division of Curriculum Development, Department of Education.

Kentucky Department of Education. (1994). Administrator standards for preparation and certification. Frankfort: Office of Teacher Education and Certification.

Kentucky Department of Education. (1998). Briefing packet: Kentucky school and district accountability cycle 3 (1994-95 to 1997-98). Frankfort: Division of Assessment.

Kentucky Department of Education. (2002). Technical report. Retrieved January 25, 2010, from http://www.education.ky.gov

Kentucky Department of Education. (2003). The Scholastic Audit: A report on school improvement in Kentucky. Frankfort: Author.

Kentucky Department of Education. (2004a). District level performance descriptions and glossary for Kentucky's Standards and Indicators for School Improvement. Frankfort: Author.

Kentucky Department of Education. (2004b). Kentucky core content tests: 2004 technical report. Retrieved January 31, 2010, from http://www.education.ky.gov

Kentucky Department of Education. (2004c). School level performance descriptors for Kentucky's Standards and Indicators for School Improvement. Frankfort: Author.

Kentucky Department of Education. (2004d). Standards and Indicators for School Improvement: A Kentucky model for student-centered accountability. Frankfort: Author.

Kentucky Department of Education. (2005). CATS interpretive guide. Retrieved June 30, 2010, from http://www.kde.state.ky.us

Kentucky Department of Education. (2006a). Accountability system. Retrieved May 26, 2010, from http://www.kde.state.ky.us

Kentucky Department of Education. (2006b). Effective Instructional Leadership Act: Technical assistance manual for instructional leaders and training program providers. Retrieved December 21, 2009, from http://www.education.ky.gov

Kentucky Department of Education. (2006c). Highly skilled educator program in Kentucky. Frankfort: Office of School Leadership and School Improvement.

Kentucky Department of Education. (2006d). Kentucky's learning goals and academic expectations. Retrieved November 20, 2009, from http://www.education.ky.gov 
Kentucky Department of Education. (2006e). Kentucky Performance Reports. Retrieved April 4, 2010, from http://www.education.ky.gov

Kentucky Department of Education. (2006f). Variance Points. Retrieved January 12, 2010, from http://www.education.ky.gov

Kentucky Department of Education. (2007). Kentucky education facts. Retrieved November 9, 2009, from http://www.education.ky.gov

Kentucky Department of Education. (n.d.). School leader's self assessment: Using the Standards and Indicators for School Improvement. Retrieved August 20, 2009, from http://www.education.ky.gov

Kentucky Education Professional Standards Board. (2006). Principal assessment: Licensure area. Retrieved January 02, 2010, from http://www.kyepsb.net

Koger, M. E., \& Thacker, A. A. (2004, June). Comparative study of Standards and Indicators for School Improvement (SISI) and Academic Index for selected elementary schools. Louisville, KY: Human Resources Research Organization.

Kouzes, J. M., \& Posner, B. Z. (1993). Credibility: How leaders gain and lose it, why people demand it. San Francisco: Jossey-Bass Publishers.

Ladd, H.F., \& Walsh, R. P. (2002). Implementing value-added measures of school effectiveness: Getting the incentives right. Economics of Education Review, 21, 117.

Lake, R. J., Hill, P., O'Toole, \& Celio, M. B. (1999). Making standards work: Active voices, focused learning. Seattle: Center on Reinventing Public Education.

Lambert, L. (1995). Towards a theory of constructivist leadership. In L. Lambert et al. (eds) The Constructivist Leader (Chicago: Teachers College Press), p. 28-51.

Lashway, L. (1999b). Holding schools accountable for achievement. ERIC Digest. (ERIC Document Reproduction Service No. ED 434 381)

Lashway, L. (2002). Trends in school leadership. ERIC Digest. (ERIC Document Reproduction Service No. ED 470 967)

LeCompte, M. D., \& Goetz, J. P. (1982). Problems of reliability and validity in ethnographic research. Review of Educational Research, 52, 31-60.

Leithwood, K. (1994). Leadership for school restructuring. Educational Administration Quarterly, 30, 498-518.

Leithwood, K., Begley, P. T., \& Cousins, J. B. (1992). Developing expert leadership for future schools. Bristol, PA: Galmer Press, Taylor \& Francis. 
Lezotte, L. W. \& Bancroft, B. A. (1985). Growing use of the effective schools model for school improvement. Educational Leadership, 42(6), 23-27.

Lindsey, C. (2007, March). State board gives its final OK to school accountability formula. Kentucky Teacher, p. 1.

Linn, R. L. (2000). Assessments and accountability. Educational Researcher, 29(2), 4-16.

Linn, R. L. (2001). Reporting school quality in standards-based accountability systems. Los Angeles: University of California at Los Angeles, Center for Research on Evaluation Standards and Student Testing. (ERIC Document Reproduction Service No. ED 455 251)

Linn, R. L. (2003). Accountability: Responsibility and reasonable expectations. Educational Researcher, 32(7), 3-13.

Lockwood, A. T. (1994). The iron triangle [Interview with Stephen K. Miller, School effectiveness research]. Focus in change, 16(Fall), 15-17.

Lumsden, R. W. (1996). Kentucky's high stakes accountability system: The explanatory power of school effectiveness variables on secondary schools in decline. Unpublished doctoral dissertation, University of Louisville.

Lyman, K. D., \& Speizer, J. J. (1980). Advancing in school administration: A pilot project for women. Harvard Educational Review, 50, 25-35.

Lynes, J. (2008). The effects of social class, social capital, parenting style, and Ogbu's oppositional identity on black college placement scores. Unpublished doctoral dissertation, University of Louisville.

Lyons, R. (2004). The influence of socioeconomic factors on Kentucky's public school accountability system: Does poverty impact school effectiveness? Education Policy Analysis Archives, 12(37). Retrieved December 15, 2009, from http://epaa.asu.edu/epaa/v12n37/

MacCoun, R., Shadish, W. R., Cook, T. D, \& Campbell, D. T. (2003). Review of experimental and quasi-experimental designs for generalized causal inference. Journal of Policy Analysis and Management, 22(2), 330-332.

Maienza, J. G. (1986). The superintendency: Characteristics of access for men and women. Educational Administration Quarterly, 22(4), 59-79.

McKinney, L. (2007). Leadership, Curriculum, Instruction, and Accountability Scores: Evidence from Kentucky Scholastic Audits. Unpublished doctoral dissertation, University of Louisville.

McKinney, L.E., Miller, S. K., \& Smith, D. C. (2007, November). Psychometric analysis of 
the curriculum, instruction, and leadership standards from Kentucky's Standards and Indicators for School Improvement. Paper presented at the annual meeting of the Mid-South Educational Research Association, Hot Spring, AR.

Mertler, C. A., \& Vannatta, R. A. (2005). Advanced and Multivariate Statistical Methods $\left(3^{\text {rd }}\right.$ ed). Glendale, CA: Pyrczak Publishing.

Meyer, J. W., \& Rowan, B. (1977). Institutionalized organizations: Formal structure as myth and ceremony. American Journal of Sociology, 83, 340-363.

Meyer, J. W., \& Rowan, B. (1978). The structure of educational organizations. In M. W. Meyer \& Associates (Eds), Environments and organizations (pp. 78-109). San Francisco: Jossey-Bass Publishers.

Miller, L. S. (1995). An American imperative: Accelerating minority educational advancement. New Haven, CT: Yale University Press.

Miller, S. K. \& Moore, B. D. (2006). Whither race? Demographic and grade level influences on reading assessment in a desegregated metropolitan district. Manuscript submitted for publication.

Mintrop, H. (2003). The limits of sanctions in low-performing schools: A study of Maryland and Kentucky schools on probation. Education Policy Analysis Archives, 11(3). Retrieved December 15, 2009 from http://epaa.asu.edu/epaa/v11n3/

Mintrop, H., \& Trujillo, T. (2005). Corrective action in low performing schools: Lessons for NCLB implementation from first-generation accountability systems. Education Policy Analysis Archives, 13(48). Retrieved December 15, 2009 from http://epaa.asu.edu/epaa/v13n48/

Moore, B. L. D. (2003). An exploration of the influence of demographic factors on individual and aggregate student achievement measurements in the Kentucky accountability system. Unpublished doctoral dissertation, University of Louisville.

Morgendollar, J. R. (1993). Introduction: The role of research on the reform of middle grades education. Elementary School Journal, 93, 443-446.

Murphy, J. (1988). Methodological, measurement, and conceptual problems in the study of instructional leadership. Educational Evaluation and Policy Analysis, 10, 117-139.

Murphy, J. (1990). The reform of school administration; Pressures and calls for change. The Reform of American Public Education in the 1980s: Perspectives and Cases, J. Murphy, ed., Berkley: McCutcheon Publishing Corporation.

Murphy, J. (1994). The changing role of the superintendency in restructuring districts in Kentucky. School Effectiveness and School Improvement, 5, 349-375. 
Murphy, J. (2004). Leadership for literacy: A framework for policy and practice. School Effectiveness and School Improvement, 15, 65-96.

Murphy, J., \& Hallinger, P. (1986). The superintendent as instructional leader: Findings from effective school districts. The Journal of Educational Administration, 24, 213236.

Murphy, J., \& Hallinger. P. (1988). Characteristics of instructionally effective school districts. Journal of Educational Research, 81, 175-181.

Murphy, J., Hallinger, P., Weil, M., \& Mitman, A. (1983). Instructional leadership: A conceptual framework. Planning and Changing, 14, 137-149.

Myers, E.,\& Murphy, J. (1987). The administrative control of high school principals by superintendents: The supervisory function. Journal of Personnel Evaluation in Education, 7, 67-79.

Napier, L. A., \& Willower, D. J. (1991). Female high school principals' perceptions of their jobs and their work interactions with males. The High School Journal, 118122.

Nachmias, D., \& Nachmias, C. (1987). Research methods in the social sciences ( ${ }^{\text {rd }}$ ed.). New York: St. Martin's Press.

National Commission on Excellence in Education. (1983). A nation at risk: The imperative for educational reform. An open letter to the American people. A report to the Nation and the Secretary of Education. Washington, DC: Superintendent of Documents, Government Printing Office. (ERIC Document Reproduction Service No. ED 226006 )

Nitko, A. J. (2001). Educational assessment of students (3rd ed.). Columbus, OH: Merrill Prentice-Hall.

Noland, K. (2007, March). SAMs project helps principals be instructional leaders. Kentucky Teacher, p. 3.

Nunnally, J. C., \& Bernstein, I. H. (1994). Psychometric theory. New York: McGraw-Hill.

Ogawa, R. T. \& Bossert, S. T. (1995). Leadership as an organizational quality. Educational Administration Quarterly, 31, 203-223.

Ortiz, F. I., \& Marshall C. (1993). Becoming a school leaders: The case of females and minorities. People and Education, 3(1), 83-110.

O'Toole, J. (1996). Leading Change: The argument for values-based leadership. New York: Ballantine Books. 
Owen, W. F. (1986). Rhetorical themes of emergent female leaders. Small Group Behavior, $17,475-486$.

Owings, W. A., Kaplan, L. S., \& Nunnery, J. (2005). Principal quality, ISLLC standards, and student achievement: A Virginia study. Journal of School Leadership, 15, 99119.

Pankratz, R. S., \& Petrosko, J. M. (Eds). (2000). All children can learn: Lessons from the Kentucky reform experience. San Francisco: Jossey-Bass.

Perkinson, H. J. (1977). The imperfect panacea: American faith in education, 1865-1976 (2nd ed.). New York: Random House.

Peterson, G. J. (1999). Demonstrated actions of instructional leaders: An examination of five California superintendents. Educational Policy Analysis Archives, 7(18). Retrieved October 28, 2004, from http://epaa.asu.edu/epaa/v7n18.html

Petrosko, J. (2000). Assessment and accountability [Electronic version]. In J. Petrosko \& J. C. Lindle (Eds.), 2000 review of research on the Kentucky Education Reform Act (pp. 1-81). Lexington: Kentucky Institute for Education Research.

Petrosko, J., \& Lindle, J. C. (EDSO). (2000). 2000 review of research on the Kentucky Education Reform Act. Lexington: Kentucky Institute for Education Research.

Pett, M. A., Lackey, N. R., \& Sullivan, J. J. (2003). Making sense of factor analysis: The use of factor analysis for instrument development in health care research. Thousand Oaks, CA: SAGE Publications.

Poggio, J. P. (2000). Statewide performance assessment and school accountability. In R. S. Pankratz \& J. M. Petrosko (Eds.), All children can learn: Lessons from the Kentucky reform experience (pp. 75-97). San Francisco: Jossey-Bass.

Prichard Committee on Academic Excellence. (1999). Gaining ground: Hard work and high expectations for Kentucky schools. Lexington, KY: Author.

Rafoth, M. A., \& Foriska, T. (2006). Administrator participation in promoting effective problem-solving teams. Remedial and Special Education, 27, 130-135.

Reeves, D. B. (2006). The learning leader: How to focus school improvement for better results. Alexandria, VA: Association for Supervision and Curriculum Development.

Roeder, P. W. (1999). Education reform and equitable excellence: The Kentucky experiment [Electronic version]. Lexington, KY: RDS Publishing.

Roeder, P. W. (2000). Education reform and equitable excellence: The Kentucky experiment (Update-February 2000), Race, poverty, and urban schools in Kentucky. Retrieved November 22, 2009, from 
http//www.uky.edu proder/kerabkupdate.pdf

Roeder, P. W. (2001). The KERA endgame: Which Kentucky schools will achieve proficiency by 2014? Retrieved November 22, 2009, from $\mathrm{http} / / \mathrm{www} \cdot u k y . e d u /{ }^{*}$ proder/keraweb.htm

Rosener, J. B. (1990). Ways women lead. Harvard Business Review, 68, 119-125.

Rosenberg, A. L., Greenfield, M. V. H., \& Dimick, J. B. (n.d.). Secondary data analysis: Using existing data to answer clinical questions. Retrieved June 21, 2006, from University of Michigan, Medical Center Web site: http://www.med.umich. edu/csp/Course\%20 materials/Summer\%202005/Rosenberg_Analysis $\% 20 \mathrm{of} \% 20$ Secondary $\% 20$ Datasets $\% 20$ Chapter\%20Final.pdf

Santos, J. (1999). Cronbach's alpha: A tool for assessing the reliability of scales. Retrieved November 23, 2010, from http://www.joe.org/joe/1999april/tt3.php

Saravia, A. (2007). Elementary parent involvement, school culture, and accountability scores: Data from Kentucky's Scholastic Audits. Unpublished doctoral dissertation, University of Louisville.

Schlechty, P. C. (2001). Shaking up the schoolhouse. San Francisco: Jossey-Bass.

Schmitt, N., \& Schechtman, S. (1987). The selection of school administrators. Journal of Personal Evaluation in Education, 3, 231-238.

Shavelson, R. J. (1996). Statistical Reasoning for the Behavioral Sciences ( ${ }^{\text {rd }}$ ed.). Boston: Allyn and Bacon.

Smith, G. (1985). Statistical reasoning. Boston. Allyn and Bacon.

Smith, M. S. (1995). Education reform in America's public schools: The Clinton agenda. In D. Ravitch (Ed.), Debating the future of American education (pp. 9-32). Washington, DC: Brookings Institution.

Smith, M. S., \& O'Day, J. (1991). Systemic school reform. In S. H. Furhman \& B. Malen (Eds.), The politics of curriculum and testing: The 1990 yearbook of the Politics of Education Association (pp. 233-267). New York: Falmer Press.

Smith, D. C. (2005). Appalachian and rural student performance differences on Kentucky's Educational assessment: Fourth grade results. Research in the Schools, 12(1), 6373.

Smith, D.C. (2006). Appalachian and rural student performance differences on Kentucky's Educational assessment: $8^{\text {th }}$ grade results. Southern Rural Sociology, 21(2), p. 47 64. 
Smith, D. C., Ennis, B. C., Saravia, A., Miller, S. K., \& Wagner, C. R. (2008, November). Psychometric analysis of the learning environment standards from Kentucky's Standards and Indicators for School Improvement. Paper presented at the annual meeting of the Mid-South Educational Research Association, Knoxville, TN.

Smith, D. C., Harvey, M. J., Hammock, J., \& Miller, S. K. (2009, October). Psychometric analysis of standards 2, 8, and 9 from Kentucky's Standards and Indicators for School Improvement. Paper presented at the 2009 National Evaluation Institute, Consortium for Research on Educational Accountability and Teacher Evaluation, Louisville, KY.

Steffy, B. E. (1993). The Kentucky education reform: Lessons for America. Lancaster, PA: Technomic Publishing.

Storey, V., \& Zellinsky, P. (1993). Beginning in school leadership: Women's perspectives on the first few years. The Canadian School Executive, 13(3), 3-5, 7-10.

Tabachnick, B. G. \& Fidell, L. S. (1996). Using multivariate statistics ( $3^{\text {rd }}$ ed.). New York: HarperCollins.

Talley, W.K. \& Keedy, J. L. (2006). Assessing school council contribution to the enabling conditions for instructional capacity building: An urban district in Kentucky. Education and Urban Society, 38(4), 419-454.

Tichy, N. M. (1997). The leadership engine: How winning companies build leaders at every level. New York: HarperCollins Publishers.

Tornsen, M. (2009). Principal leadership, national responsibilities and successful school outcomes. ISEA, 37(3), 37-52.

Tyack, D. B. (1974). The one best system: A history of American urban education. Cambridge, MA: Harvard University Press.

Vogt, W. P. (2005). Dictionary of statistics \& methodology (3rd ed). Thousand Oaks, CA: Sage.

Wagner, C. R. (1998, November). Improving schools through the administration and analysis of school culture audits. Paper presented at the annual meeting of the MidSouth Educational Research Association, New Orleans.

Waters, T., \& Kingston, S. (2005, September/October). The standards we need. Leadership, 14-39.

Waters, J. T., Marzano, R. J., \& McNulty, B. A. (2005, April). McREL's school leadership that works framework: Applications and implications. Paper presented at the annual meeting of the Association for Supervision and Curriculum Development, Orlando, FL. 
Webb, E. J., Campbell, D. T., Schwartz, R. D., \& Sechrest, L. (1966). Unobtrusive measures: Nonreactive research in the social sciences. Chicago: Rand McNally \& Company.

Weiss, I. R., \& Pasley, J. D. (2004). What is high-quality instruction? Educational Leadership, 6l(5), 24-28.

Weiss, I. R., Pasley, J. D., Smith, P. S., Banilower, E. R., \& Heck, D. J. (2003). Looking inside the classroom: $A$ study of $K-12$ mathematics and science education in the United States. Chapel Hill, NC: Horizon Research.

Wilhoit, G. (2003). Message from the Commissioner. In The Office of Leadership and School Improvement, Division of School Improvement, The Scholastic Audit (p. 3). Frankfort: Kentucky Department of Education.

Williams, F. (1992). Reasoning with statistics: How to read quantitative research ( $4^{\text {th }}$ ed.). Fort Worth, TX: Harcourt Brace Jovanovich.

Winter, P. A., \& Morgenthal, J. R. (2001). The impact of school achievement on principal recruitment in a reform environment. Paper presented at the annual meeting of the University Council for Educational Administration, Cincinnati. 
APPENDIX A

STANDARDS AND INDICATORS FOR SCHOOL IMPROVEMENT 


\section{Appendix A}

Standards and Indicators for School Improvement

These Standards and Indicators for School Improvement represent guidelines for schools to maximize potential for all students by defining professional standards in these areas, organized into three broad categories--Academic Performance, Learning Environment, and Efficiency (Division of School Improvement, 2003). Each of the nine standards includes a number of indicators, ranging from 5 to 16 . The indicators are listed in KDE (2004d), Standards and Indicators for School Improvement: A Kentucky Model for Student-Centered Accountability.

Academic Performance

Standard 1 (Curriculum): The school develops and implements a curriculum that is rigorous, intentional, and aligned to state and local standards.

Standard 2 (Classroom Evaluation/Assessment): The school uses multiple evaluation and assessment strategies to continuously monitor and modify instruction to meet student needs and support proficient student work.

Standard 3 (Instruction): The school's instructional program actively engages all students by using effective, varied and research-based practices to improve student academic performance standards.

Learning Environment

Standard 4 (School Culture): The school/district functions as an effective learning community and supports a climate conducive to performance excellence.

Standard 5 (Student, Family and Community Support): The school/district works with families and community groups to remove barriers to learning in an effort to meet the intellectual, social, career, and developmental needs of students.

Standard 6 (Professional Growth, Development and Evaluation): The school/district provides research-based, results driven professional development opportunities for staff and implements performance evaluation procedures in order to improve teaching and learning.

Efficiency 
Standard 7 (Leadership): School/district instructional decisions focus on support for teaching and learning, organizational direction, high performance expectations, creating a learning culture, and developing leadership capacity.

Standard 8 (Organizational Structure and Resources): The organization of the school/district maximizes use of time, all available space and other resources to maximize teaching and learning and support high student and staff performances.

Standard 9 (Comprehensive and Effective Planning): The school/district develops, implements and evaluates a comprehensive school improvement plan that communicates a clear purpose, direction and action plan focused on teaching and learning. 
APPENDIX B

INTERSTATE SCHOOL LEADER LICENSURE CONSORTIUM STANDARDS 


\section{Appendix B}

Interstate School Leader Licensure Consortium Standards

The core curriculum for leadership of schools for the Commonwealth of Kentucky is grounded in the Standards and Indictors for School Improvement (SISI). With references and relationships to the standards of the Interstate School Leadership Licensure Consortium (ISLLC), the goals of The Kentucky Board of Education, a specific focus on SISI Standard 7 (Leadership), and the Technology Standards for School Administrators, these curriculum documents provide a map by which Kentucky school leaders can move toward greater effectiveness as instructional leaders and increasing gains in student achievement (KDE, n.d.). The ISLLC Standards are listed below. A complete list of ISLLC indicators for each standard may be found on the KDE website or by consulting the Council of Chief State School Officers (1996).

Standard 1: A school administrator is an educational leader who promotes the success of all students by facilitating the development, articulation, implementation, and stewardship of a vision of learning that is shared and supported by the school community.

Standard 2: A school administrator is an educational leader who promotes the success of all students by advocating, nurturing, and sustaining a school culture and instructional program conducive to student learning and staff professional growth.

Standard 3: A school administrator is an educational leader who promotes the success of all students by ensuring management of the organization, operations, and resources for a safe, efficient, and effective learning environment.

Standard 4: A school administrator is an educational leader who promotes the success of all students by collaborating with families and community members, responding to diverse community interests and needs, and mobilizing community resources.

Standard 5: A school administrator is an educational leader who promotes the success of all students by acting with integrity, fairness, and in an ethical manner.

Standard 6: A school administrator is an educational leader who promotes the success of all students by understanding, responding to, and influencing the larger political, social, economic, legal, and cultural context. 
APPENDIX C

SEVENTEEN LEVERAGE POINTS FROM 2000-2001 SCHOLASTIC AUDIT 


\section{Appendix C}

Seventeen Leverage Points from 2000-2001 Scholastic Audit

The first round of scholastic audits/reviews (2000-2001) produced seventeen indicators designated as Leverage Points: indicators where results varied greatly from successful schools to Level 3 assistance schools. After the audits/reviews were completed in 2002-2003, the term "Leverage Point" was changed to "Variance Point" to describe more accurately the meaning of the term (Division of School Improvement, 2003). Listed below are the seventeen leverage indicators based on the data obtained from the $2000-2001$ scholastic audits and reviews.

Standard 1, Curriculum

1.1d. There is evidence of vertical communication with an intentional focus on key curriculum transition points within grade configurations (e.g., from primary to middle and middle to high).

Standard 2, Assessment

2.1d. Test scores are used to identify curriculum gaps.

2.1h. Samples of student work are analyzed to inform instruction, revise curriculum and pedagogy, and obtain information on student progress.

Standard 3, Instruction

3.1b. Instructional strategies and learning activities are aligned with the district, school, and state learning goals and assessment expectations for student learning.

Standard 4, School Culture

4.1f. The school intentionally assigns staff to maximize opportunities for all students to have access to the staff's instructional strengths.

Standard 5, Student, Family and Community Support

5.1b. Structures are in place to ensure that all students have access to all curriculum (e.g., school guidance, Family Resource/Youth Services Centers, Extended School Services). 
5.1d. Students are provided with a variety of opportunities to receive additional assistance to support their learning beyond the initial classroom instruction.

Standard 6, Professional Growth, Development and Evaluation

6.1b. The school has an intentional plan for building instructional capacity through ongoing professional development.

6.2c. The school/district effectively uses the employee evaluation and the individual professional growth plan to improve staff proficiency.

6.2e. The school/district improvement plan identifies specific instructional leadership needs, has strategies to address them, and uses the Effective Instructional Leadership Act requirements as a resource to accomplish these goals.

6.2f. Leadership uses the evaluation process to provide teachers with the follow-up and support to change behavior and instructional practice.

Standard 7, Leadership

7.1c. There is evidence that all administrators have a growth plan focused on the development of effective leadership skills.

7.1d. There is evidence that the school/district leadership team disaggregates data for use in meeting the needs of a diverse population, communicates the information to school staff and incorporates the data systematically into the school's plan.

Standard 8, Organizational Structure and Resources

8.2b. The school/district budget reflects decisions made about discretionary funds and resources are directed by an assessment of need or a required plan, all of which considers appropriate data.

8.2c. School councils and school boards analyze funding and other resource requests to ensure the requests are tied to the school's plan and identified priority needs.

Standard 9, Comprehensive and Effective Planning

9.3b. The school/district analyzes their students' unique learning needs.

9.6b. The school evaluates the degree to which it achieves the goals and objectives for student learning set by the plan. 
APPENDIX D

TWENTY-SEVEN VARIANCE POINTS FROM 2002-2003 SCHOLASTIC AUDIT 


\section{Appendix D}

Twenty-seven Variance Points from 2002-2003 Scholastic Audit

Listed below are the twenty-seven indicators that were found to be variance points based on the data obtained from the 2002-2003 scholastic audits and reviews (KDE, 2006f).

Standard 1, Curriculum

1.1g. The curriculum provides access to a common academic core for all students.

Standard 2, Assessment

2.1d. Test scores are used to identify curriculum gaps.

2.1h. Samples of student work are analyzed to inform instruction, revise curriculum and pedagogy, and obtain information on student progress.

Standard 3, Instruction

3.1b. Instructional strategies and learning activities are aligned with the district, school, and state learning goals and assessment expectations for student learning.

3.1d. Teachers demonstrate the content knowledge necessary to challenge and motivate students to high levels of learning.

Standard 4, School Culture

4.1a. There is leadership support for a safe, orderly, and equitable learning environment (e.g., culture audits/school opinion surveys).

4.1b. Leadership creates experiences that foster the belief that all children can learn at high levels in order to motivate staff to produce continuous improvement in student learning.

4.1c. Teachers hold high expectations for all students academically and behaviorally; this is evidenced in their practices.

4.1d. Teachers and non-teaching staff are involved in both formal and informal decisionmaking processes regarding teaching and learning.

4.1e. Teachers recognize and accept their professional role in student success and failure. 
4.1f. The school intentionally assigns staff to maximize opportunities for all students to have access to the staff's instructional strengths.

4.1h. There is evidence that the teachers and staff care about students and inspire their best efforts.

4.1i. Multiple communication strategies and contexts are used for the dissemination of information to all stakeholders.

4.1j. There is evidence that student achievement is highly valued and publicly celebrated (e.g., displays of student work, assemblies).

4.1k. The school/district provides support for the physical, cultural, socio-economic, and intellectual needs of all students which reflects a commitment to equity and an appreciation of diversity.

Standard 5, Student, Family and Community Support

5.1a. Families and the community are active partners in the educational process and work together with the school/district staff to promote programs and services for all students.

5.1d. Students are provided with a variety of opportunities to receive additional assistance to support their learning beyond the initial classroom instruction.

Standard 6, Professional Growth, Development and Evaluation

6.1c. Staff development priorities are set in alignment with the goals for student performance and the individual growth plans of staff.

6.1f. Professional development planning shows a direct connection to and analysis of student achievement data.

6.2c. The school/district effectively uses the employee evaluation and the individual professional growth plan to improve staff proficiency.

6.2d. Leadership provides and implements a process of personnel evaluation which meets or exceeds standards set in statute and regulation.

Standard 7, Leadership

7.1k. There is evidence that the principal demonstrates leadership skills in the areas of academic performance, learning environment, and efficiency.

Standard 8, Organizational Structure and Resources

8.1a. There is evidence that the school is organized to maximize use of all available resources to support high student and staff performance. 
8.1c. The instructional and non-instructional staff are allocated and organized based on the learning needs of all students.

8.1d. There is evidence that the staff makes efficient use of instructional time to maximize student learning.

8.1f. The schedule is intentionally aligned with the school's mission and designed to ensure that all staff provide quality instructional time (e.g., flex time, organization based on the developmental needs of students, interdisciplinary units, etc.).

Standard 9, Comprehensive and Effective Planning

$9.4 \mathrm{~b}$. The school/district goals for building and strengthening the capacity of the school/district instructional and organizational effectiveness are defined. 
APPENDIX E

SIX COMMON VARIANCE POINTS 


\title{
Appendix E
}

\author{
Six Common Variance Points
}

The following six Common Variance Points were found in both the 2001-2002 and 2002-2003 scholastic audits and reviews (Division of School Improvement, 2003).

Standard 2, Assessment

2.1d. Test scores are used to identify curriculum gaps.

2.1h. Samples of student work are analyzed to inform instruction, revise curriculum and pedagogy, and obtain information on student progress.

Standard 3, Instruction

3.1b. Instructional strategies and learning activities are aligned with the district, school, and state learning goals and assessment expectations for student learning.

Standard 4, School Culture

4.1f. The school intentionally assigns staff to maximize opportunities for all students to have access to the staff's instructional strengths.

Standard 5, Student, Family and Community Support

5.1d. Students are provided with a variety of opportunities to receive additional assistance to support their learning beyond the initial classroom instruction.

Standard 6, Professional Growth, Development and Evaluation

6.2c. The school/district effectively uses the employee evaluation and the individual professional growth plan to improve staff proficiency. 


\section{APPENDIX F}

\section{ELEVEN COMMON VARIANCE POINTS}




\section{Appendix F}

\section{Eleven Common Variance Points}

The standard indicators that consistently emerged as Common Variance Points in all three accountability cycles (2000-2001, 2002-2003, 2004-2005) are listed below (KDE, 2006f). It should be noted that after the last cycle of audits, $\mathrm{KDE}$ changed the criteria for determining when the audit scores on a specific indicator were significantly different when compared across high and low achieving schools. This explains the discrepancy in the Common Variance Points between Appendix E and Appendix F.

Standard 2, Assessment

2.1g. Implementation of the state-required Assessment and Accountability Program is coordinated by school and district leadership.

Standard 4, School Culture

4.1a. There is leadership support for a safe, orderly, and equitable learning environment (e.g., culture audits/school opinion surveys).

4.1h. There is evidence that the teachers and staff care about students and inspire their best efforts.

Standard 6, Professional Growth, Development and Evaluation

6.2a. The school/district provides a clearly defined evaluation process.

6.2b. Leadership provides the fiscal resources for the appropriate professional growth plan to improve staff proficiency.

6.2d. Leadership provides and implements a process of personnel evaluation which meets or exceeds standards set in statute and regulation.

Standard 7, Leadership

7.1c. There is evidence that all administrators have a growth plan focused on the development of effective leadership skills.

7.1e. Leadership ensures all instructional staff has access to curriculum related materials and the training necessary to use curricular and data resources relating to the learning goals 
for Kentucky public schools.

7.1g. Leadership plans and allocates resources, monitors progress, provides organizational infrastructure and removes barriers in order to sustain continuous school improvement.

7.1h. The school/district leadership provides the organizational policy and resource infrastructure necessary for the implementation and maintenance of a safe and effective learning environment.

7.1k. There is evidence that the principal demonstrates leadership skills in the areas of academic performance, learning environment, and efficiency. 


\section{APPENDIX G}

HUMAN SUBJECTS APPROVAL 


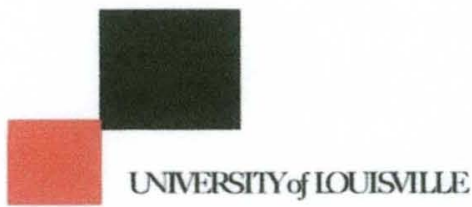

\begin{tabular}{l} 
INSTITUTIONAL REVIEW BOARDS \\
\hline University of Louisville \\
MedCenter One, Suite 200 \\
$501 \mathrm{E}$. Broadway \\
Louisville, Kentucky 40202-1798 \\
Office: $\quad 502-852-5188$ \\
Fax: $\quad 502-852-2164$
\end{tabular}

\author{
To: $\quad$ Miller, Stephen \\ From: The University of Louisville Institutional Review Board (IRB) \\ Date: $\quad$ Monday, October 18, 2010 \\ Subject: IRB Correspondence \\ Tracking \#: 10.0524 \\ Title: $\quad$ LEADERSHIP, CURRICULUM, INSTRUCTION, AND ACCOUNTABILITY \\ SCORES: EVIDENCE FROM KENTUCKY SCHOLASTIC AUDITS
}

This study was reviewed on 10/15/2010 and determined by the chair of the Institutional Review Board that the study is exempt according to 45 CFR 46.101(b) under

category (4) Research involving the collection or study of existing data, documents, records, pathological specimens, or diagnostic specimens, if these sources are publicly available or if the information is recorded by the investigator in such a manner that subjects cannot be identified, directly or through identifiers linked to the subjects. The study is exempt only if information that could identify subjects is not recorded.

Since this study has been found to be exempt, no additional reporting, such as submission of Progress Reports for continuation reviews, is needed. If your research focus or activities change, please submit a Study Amendment Request Form to the IRB for review to ensure that the study still meets exempt status. Best wishes for a successful study. Please send all inquires and electronic revised/requested items to our office email address at hsppofc:gwise.louisville.edu.

\title{
Pete $m$ Lusach
}

Board Designee: Quesada, Peter 




\begin{tabular}{l}
\hline INSTITUTIONAL REVIEW BOAROS \\
\hline University of Louisville \\
MedCenter One, Suite 200 \\
501 E. Broadway \\
Louisville, Kentucky 40202-1798 \\
Office: $\quad 502-852-5188$ \\
Fax: $\quad 502-852-2164$
\end{tabular}

Expedited - Amendment - Exemption

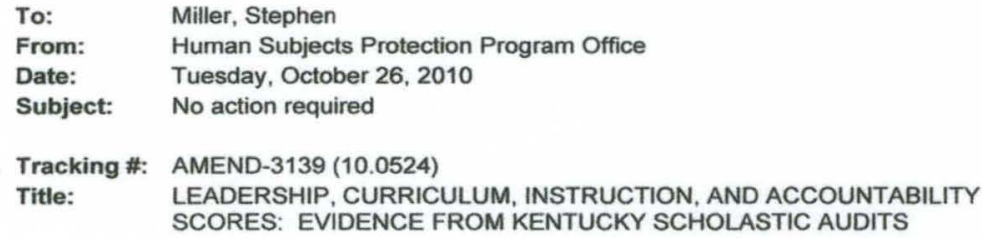

Determination Date: $10 / 26 / 2010$

The following items have been received by the Human Subjects Protection Program Office and were reviewed by the chair/vice-chair of the Institutional Review Board (IRB):

\section{- AMEND-3139}

The modifications include:

- The addition of Rebecca Todd to the study as the primary contact.

This information has been reviewed and determined by the chair of the Institutional Review Board (IRB) not to change the exempt status of this study. Thank you for keeping us informed of your study.

Thank you.

\section{Pte $m$ Lusach}

Board Designee: Quesada, Peter Letter Sent By: Perkins, Erin, 10/26/2010 1:00 PM

Full Accreditation since June 2005 by the Association for the Accreditation of Human Research Protection Programs, Inc. 


\section{요요 \\ WKU.}

A L.EADING AMERICAN UNIVT RSITY WITH INTERNATIONAL REACH

HUMAN SUBBLETS REVIFW BOAR

In future correspondence, please refer to HS11-077, October 19, 2010

Rebecca Curry Todd

c/o Dr. Miller

Dr. Smith

Education

WXU

Rebecca Curry Todd:

Your rescarch project, Leadership, Curricultum, Instruction, and Accountability Scores: Evidence from Kentucky Scholastic Audits, was reviewed by the HSRB and it has been determined that risks to subjects are: (1) minimized and reasonable; and that (2) research procedures are consistent with a sound research design and do not expose the subjects to unnecessary risk. Reviewers determined that: (1) benefits to subjecis are cansidered along with the importance of the topic and that outcomes are reasonable; (2)

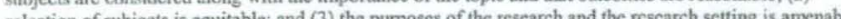

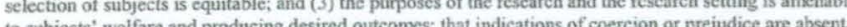
to subjects "welfare and producing desired on

1. In addition, the IRB found that you need to orient participants as follows: (1) signed informed consent is not required; (2) Provision is made for collecting, using and storing data in a manner that protects the safety and privacy of the subjects and the confidentiality of the data. (3) Appropriate safeguards are included to proteet the rights and welfare of the subjects.

\section{This project is therefore approved at the Exempt from Full Board Review Level.}

2. Please note that the institution is not responsible for any actions regarding this protocol before approval. If you expand the project at a later date to use other instruments please re-apply. Copies of your request for human subjects review, your application, and this approval, are maintained in the Office of Sponsored Programs at the above address. Please report any changes to this approved protocol to this office. A Continuing Review protocol will be sent to you in the future to determine the status of the project. Also, please use the stamped approval forms to assure participants of compliance with The Office of Human Research Profections regulations.

Sincerely,

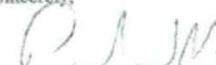

Paul J. Mooney, M.S.T.M

Compliance Coordinato

Coffice of Research

Western Kentucky University

ce: HS file number Todd HS11-07



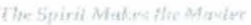

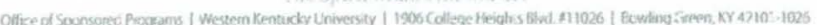



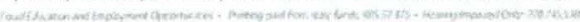


APPENDIX H

SUPPLEMENTAL TABLES 
Table H1

Structure and Patten Matrix Coefficients from Principal Component Analysis of Standard 7 $(N=83)$

\begin{tabular}{|c|c|c|c|c|}
\hline \multirow[b]{2}{*}{ Indicators $^{\mathbf{a}}$} & \multicolumn{2}{|c|}{ Factor Structure Matrix } & \multicolumn{2}{|c|}{ Factor Pattern Matrix } \\
\hline & Comp 1 & Comp 2 & Comp 1 & Comp 2 \\
\hline $7.1 . \mathrm{i}$ & .860 & .239 & .953 & -.201 \\
\hline 7.1.j & .783 & .312 & .812 & -.062 \\
\hline 7.1.b & .739 & .527 & .630 & .236 \\
\hline 7.1.d & .696 & .459 & .616 & .175 \\
\hline 7.1.a & .620 & .398 & .555 & .142 \\
\hline 7.1.e & .616 & .658 & .397 & .474 \\
\hline $7.1 . \mathrm{k}$ & .586 & .746 & .308 & .604 \\
\hline 7.1.f & .445 & .677 & .168 & .600 \\
\hline $7.1 . \mathrm{g}$ & .506 & .832 & .155 & .761 \\
\hline 7.1.h & .245 & .745 & -.100 & .711 \\
\hline 7.1.c & .228 & .665 & -.126 & .803 \\
\hline
\end{tabular}

${ }^{\mathrm{a}} \mathrm{KDE}$ (2004b, pp. 32-33); wording for indicators is in Table 4. 
Table H2

Structure and Pattern Matrix Coefficients from Principal Component Analysis of Standard $1(N=83)$

\begin{tabular}{|c|c|c|c|c|}
\hline \multirow[b]{2}{*}{ Indicators ${ }^{\mathrm{a}}$} & \multicolumn{2}{|c|}{ Factor Structure Matrix } & \multicolumn{2}{|c|}{ Factor Pattern Matrix } \\
\hline & Comp 1 & Comp 2 & Comp 1 & Comp 2 \\
\hline 1.1.b & .883 & .279 & .919 & -.089 \\
\hline 1.1.c & .867 & .374 & .855 & .031 \\
\hline 1.1.d & .823 & .300 & .837 & -.035 \\
\hline 1.1.a & .705 & .665 & .522 & .456 \\
\hline 1.1.f & .659 & .681 & .460 & .497 \\
\hline $1.1 . \mathrm{g}$ & .415 & .748 & .137 & .693 \\
\hline 1.1.e & .173 & .767 & -.160 & .831 \\
\hline
\end{tabular}




\section{CURRICULUM VITAE}

NAME:

Rebecca Curry Todd

ADDRESS:

378 Woodsbend Road

Elizabethtown, Kentucky 42701

EDUCATION: $\quad$ B.A., Public Relations

Western Kentucky University 1980

B.S., Biology

Western Kentucky University 1995

M.A.Ed., Biology

Western Kentucky University 1997

Rank I, K12 Principal Certification

\& Superintendent Certification

Western Kentucky University

1998

AWARDS:

Kentucky Colonel

Honorary Commissioner of Agriculture

Kentucky Secondary Conservation Teacher of the Year 1999

Ted Jones Prospecting Award

2009

Edward Jones Partners Award

2010 